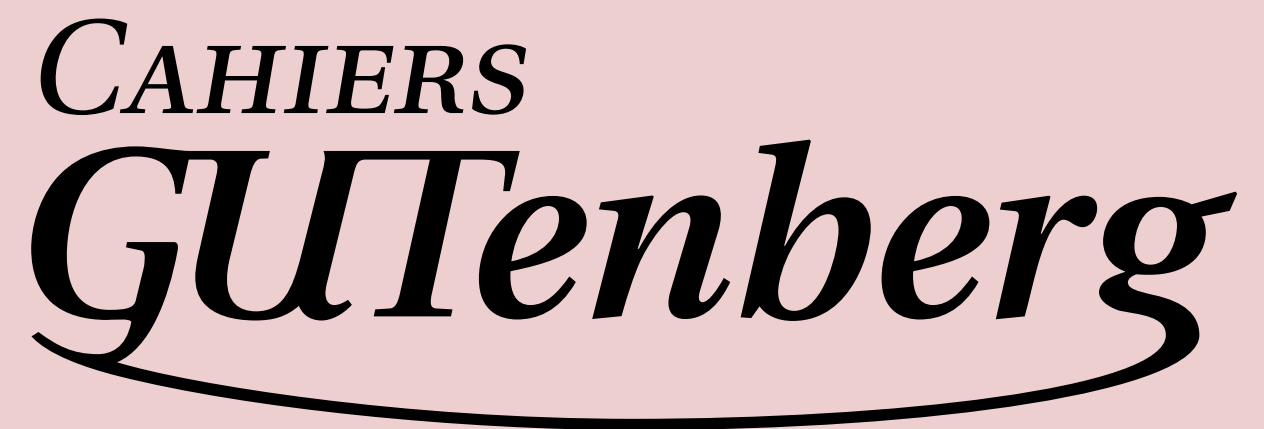

\title{
@ METAPOST RACONTÉ AUX PIÉTONS
}

\section{d Yves Soulet}

Cahiers GUTenberg, no 52-53 (2009), p. 5-117.

$<$ http://cahiers.gutenberg.eu.org/fitem?id=CG_2009__52-53_5_0>

๑) Association GUTenberg, 2009, tous droits réservés.

L'accès aux articles des Cahiers GUTenberg

(http://cahiers.gutenberg.eu.org/),

implique l'accord avec les conditions générales

d'utilisation (http://cahiers.gutenberg.eu.org/legal.html).

Toute utilisation commerciale ou impression systématique

est constitutive d'une infraction pénale. Toute copie ou impression de ce fichier doit contenir la présente mention de copyright. 



\title{
G METAPOST RACONTÉ AUX PIÉTONS
}

\section{a Yves Soulet}

\begin{abstract}
RÉSumÉ. - Ce texte est une introduction à METAPOST destinée aux utlisateurs potentiels souhaitant tester en peu de temps ce puissant outil graphique. Il est écrit dans un langage simple, accessible aux non familiers du langage de l'informatique et propose une progression motivante accompagnée de très nombreux exercices.

Aвstract. - This is a manual for getting started with the powerful graphic language METAPOST. It is written in a most accessible manner for those not so familiar with computer programming. It comes with a load of exercises and illustrations, which are each carefully explained. The example files are available for download on the Cahiers' website.

Note. - Cet manuel est disponible en version électronique sur le site de la revue cahiers.gutenberg.eu.org. Outre le PDF des pages qui suivent, qui permet de copier le code décrit et de voir les détails des figures, on peut y récupérer les fichiers METAPOST utilisés pour les exemples.
\end{abstract}

\section{INTRODUCTION}

Lors de la publication du Manuel de prise en main pour TikZ (Cahiers GUTenberg, $\mathrm{n}^{\circ} 50$, avril 2008), il y a eu une assez longue discussion sur la liste GUT où l'on a pu constater que beaucoup d'utilisateurs de $\mathrm{L}_{\mathrm{TE}} \mathrm{X}$ souhaitaient savoir quel était le meilleur outil pour la production de figures de qualité irréprochable (et bien entendu avec des lettrages composés «à la $\mathrm{LT}_{\mathrm{E}} \mathrm{X}$ »). D' autres, qui utilisent couramment un ou plusieurs de ces outils ont expliqué les avantages et les inconvénients des uns et des autres. L'un de ces outils (et ses dérivés bien entendu) se distingue bien des autres : on veut tracer une tangente à un cercle passant par un point extérieur donné; PSTRICKs et TikZ tracent cette tangente si on leur donne les coordonnées du point de contact (ou d'un autre deuxième 
point) ; METAPOST calcule les coordonnées de ce point de contact si " on sait le lui demander »; là se trouve la différence!

Son histoire commence au début des années 80 où Donald Knuth conçoit METAFONT, un langage destiné à produire des caractères; c'est ainsi qu'il a pu créer les fontes CM. Ce langage permet de réaliser le dessin des caractères et, dans une deuxième étape, de produire sa rastérisation (c'est-à-dire le choix des pixels à noircir pour obtenir le meilleur rendu à l'impression).

Par la suite apparaît le langage PostScript et les imprimantes professionnelles sont équipées d'un processeur (l'interpréteur PostScript) qui traite parfaitement le problème de la rastérisation en prenant en compte les caractéristiques spécifiques de la machine pour laquelle il a été conçu (résolution et beaucoup d'autres paramètres). En conséquence, au début des années 90, est créé METAPOST qui est un langage dérivé de METAFONT; quelques primitives ont été ajoutées, d'autres on été abandonnées. Au lieu de produire des objets graphiques sous forme d'une liste de coordonnées de pixels à noircir, METAPOST produit le code PostScript de ces objets (d'où l'abandon de toutes les primitives concernant la rastérisation).

En 1992, John Hobby publie un document, A User's Manual for META$P O S T$, dont la traduction française, Un manuel de l'utilisateur pour METAPOST par Jean-Côme Charpentier et Pierre Fournier, est publiée à son tour par les Cahiers GUTenberg, no 41 , novembre 2001. Ce manuel est de lecture assez difficile pour les utilisateurs de $\mathrm{LAT}_{\mathrm{E}} \mathrm{X}$ qui ne sont pas familiers avec le langage de la discipline informatique. Un manuel du type "pour les piétons ${ }^{1}$ » devrait aider et encourager les utilisateurs potentiels dans leur début en METAPOST : c'est le but du présent document.

Ces quelques pages n'ont pas la rigueur du manuel de John Hobby; elles n'ont pas la prétention de les remplacer; en effet, certaines spécificités du langage parmi les plus délicates ne sont pas abordées (ou sont simplement citées avec une petite «illustration»). Elles présentent, dans un langage simple et accompagné d'exercices avec leur code complet,

1. L'auteur de ces lignes avait été émerveillé par le petit livre Feynman diagrams for pedestrians qui lui avait facilité ses débuts en électrodynamique quantique.

Cahiers GUTenberg no 52-53-Octobre 2009 
l'essentiel permettant au débutant de réaliser des figures assez complexes. Toutes les primitives et toutes les macros (définies dans le fichier plain .mp et dans quelques autres fichiers cités en temps utile) sont illustrées au fur et à mesure par des exemples conçus pour faire découvrir progressivement la structure du langage.

L’originalité de ce document réside dans le fait que le débutant est invité à faire les exercices et en voir les résultats immédiatement avec une visionneuse PostScript (Gsview, GHostview, etc.).

Les références $[\mathrm{xxx}]$ se rapportent aux pages du no 41 des Cahiers GUTenberg où se trouve, en plus du manuel METAPOST déjà cité et de l'article du même auteur concernant la production de graphiques (extension GRAPH), un petit article de Fabrice Popineau consacré au côté pratique de l'utilisation de METAPOST. Les références du type [M xxx] pointent vers des pages du livre de référence de METAFONT ${ }^{2}$ et celle du type $[\mathrm{H} \mathrm{xx}]$ vers les pages du document original de John Hobby mpgraph.pdf disponible dans les distributions $\mathrm{T}_{\mathrm{E}} \mathrm{X}$.

Les trois premiers chapitres sont conçus pour permettre aux débutants d'acquérir un savoir faire suffisant pour réaliser des figures relativement complexes. Le quatrième chapitre est consacré à la «machinerie " METAPOST, c'est-à-dire à la structure du langage avec ses variables, ses opérateurs, ses boucles conditionnelles, ses commandes de bifurcations, et aux règles de construction des macros utilisateur. Le cinquième chapitre expose la construction des nœuds et des liaisons internœuds en vue de produire des organigrammes, des algorithmes, etc. Le sixième chapitre est destiné à la construction de graphiques, ce que les physiciens appellent les courbes, à l'aide d'une extension de fichier graph.mp; le point fort de cette extension est une mise à l'échelle automatique des données pour que le graphique ait les dimensions imposées par avance. Quelques macros de l'auteur de ces lignes permettent de «relooker» ces graphiques tout en conservant les avantages de l'extension citée. Le septième et dernier chapitre est très court; il explique comment ajouter des lettrages « à la $\mathrm{LAT}_{\mathrm{E}} \mathrm{X}$ » dans les figures et propose une méthode de travail pour les produire.

2. Donald Knuth, METAFONTBook, Addison Wesley, 1986

METAPOST raconté aux piétons 
METAPOST mérite bien plus que ce petit document qui ne veut qu'être une aide pour débuter avec une progression motivante! L'objectif de ces pages est de permettre à tous ceux qui sont totalement absorbés par leurs tâches quotidiennes d'acquérir une certaine pratique de METAPOST sans y consacrer trop de temps. Après ce début, ils auront la capacité nécessaire pour rechercher et évaluer les extensions METAPOST disponibles qui conviennent le mieux à leurs objectifs... en ajoutant peut-être quelques macros complémentaires très simples.

L'index, abondant, contient toutes les primitives et toutes les macros avec les numéros de page où elles sont introduites puis utilisées; il ne reprend pas les titres de la table des matières; au lecteur d'utiliser l'index et la table pour ses recherches.

\section{TABLE DES MATIÈRES}

Introduction

Chapitre 1. Coordonnées et lettrages 11

1. Coordonnées cartésiennes $\quad 12$

2. Coordonnées polaires 14

3. Lettrage direct 14

Chapitre 2. Tracé de lignes brisées $\quad 17$

1. Syntaxe de base du tracé 17

2. Options de tracé $\quad 19$

2.1. Épaisseur du trait 19

2.2. Terminaisons et jonctions de traits 21

2.3. Pointillés et traitillés $\quad 22$

2.4. Flèches 23

2.5. Couleurs 24

3. Compléments 25

3.1. Résolution d'équations linéaires 25

3.2. Intersection de droites $\quad 25$

3.3. Précisions de certains points de syntaxe 26

3.4. Préambule du fichier des figures 27

Chapitre 3. Tracé de lignes courbes 29

1. Un tracé élémentaire pour débuter 29

2. Courbes de Bézier cubiques 31

Cahiers GUTenberg no 52-53-Octobre 2009 
3. Différentes possibilités de tracés de courbes 32

3.1. Tracé avec les seuls points guides 32

3.2. Tracé en fixant les tangentes aux points guides 32

3.3. Modification du tracé : tension et courbure 33

4. Gestion des courbes $\quad 35$

4.1. Découpage des courbes $\quad 35$

4.2. Intersections de courbes $\quad 36$

4.3. Construction directe des zones d'intersection 38

4.4. Tangentes et normales aux courbes 40

5. Compléments 42

5.1. Cercles et arcs de cercles $\quad 42$

5.2. Autres commandes concernant les courbes 43

5.3. Explications concernant des points de syntaxe 43

Chapitre 4. La machinerie METAPOST $\quad 47$

1. Opérateurs $\quad 47$

2. Variables $\quad 48$

2.1. Type nombre 49

2.2. Type paire 50

2.3. Type couleur 50

2.4. Type transformation 51

2.5. Type chemin 53

2.6. Type chaîne 54

2.7. Type booléen $\quad 55$

2.8. Type dessin 55

2.9. Type plume 57

3. Variable et équations; variables internes et affectations 57

3.1. Variables (ordinaires) 57

3.2. Variables internes $\quad 58$

3.3. Remarques concernant les affectations 58

4. Commandes et macros $\quad 59$

4.1. Commandes 59

4.2. Macros de base $\quad 60$

4.3. Construction de macros 61

5. Boucles et tests $\quad 63$

5.1. Boucles 63

5.2. Tests 64 
Chapitre 5. Boîtes et liaisons : organigrammes et algorithmes

1. Boîtes rectangulaires

1.1. Création de la boîte 66

1.2. Tracé de la boîte 66

1.3. Améliorations : fond et contour 68

2. Autres formes : arrondies, circulaires, etc. 69

3. Macros élémentaires à la demande $\quad 70$

4. Liaisons entre les boîtes 73

4.1. Extrémités des liaisons $\quad 73$

4.2. Liaisons à un seul segment $\quad 74$

4.3. Liaisons à deux segments $\quad 75$

4.4. Liaisons à trois segments et plus 76

5. Mettre des labels (ou lettrages) sur les liaisons $\quad 78$

Chapitre 6. Tracé de courbes 81

1. Tracés par défaut $\quad 82$

2. Types de tracés disponibles $\quad 83$

3. Domaines de variation et types de coordonnées 87

4. Cadre (ou axes) et graduations (ticks et labels) 90

5. Légende des axes et labels supplémentaires 91

6. Difficultés dues aux grands nombres 93

7. Tracé de courbes METAPOST «relookées» 96

Chapitre 7. METAPOST et $\mathrm{T}_{\mathrm{E}} \mathrm{X}$

1. Étapes du lettrage des figures 101

1.1. Production des fichiers tex et dvi des lettrages 102

1.2. Production du fichier mpx des fichiers de figure lettrés $\quad 103$

1.3. Automatisation des opérations de lettrage 103

2. Lettrage en $\mathrm{IAT}_{\mathrm{E}} \mathrm{X}$ de quelques figures 104

3. Lettrage en $\mathrm{ATT}_{\mathrm{E} X} \mathrm{X}$ de quelques graphiques 106

4. METAPOST et TEX, tout à la fois! 108

$\begin{array}{ll}\text { Conclusion } & 111\end{array}$

$\begin{array}{ll}\text { Index } & 113\end{array}$

Cahiers GUTenberg no 52-53-Octobre 2009 


\section{CHAPITRE 1 \\ Coordonnées et lettrages}

Ce premier (et tout petit) chapitre est destiné à introduire les notations de base pour représenter les points du plan avec leurs coordonnées. Dans les premiers exemples, on se donne des points et on les relie entre eux par des segments que l'on trace avec la commande draw, commande qui sera reprise en détail au chapitre 2. Une grille, axespapiermilli, d'un pas de $1 \mathrm{~mm}$ et des axes permettent de visualiser facilement le résultat des commandes. Dès ce premier chapitre, on commence à exploiter toutes les propriétés de METAPOST qui est un langage informatique à part entière avec variables, opérateurs, commandes, fonctions prédéfinies et définies par l'utilisateur, boucles de commandes, etc.

Tout au long de ce petit manuel, le lecteur est invité à refaire les exemples, à les modifier et à en imaginer d'autres. Pour faciliter ce travail, il est parfois important de pouvoir lettrer les figures des exemples : pour cela, on délaisse provisoirement $\mathrm{T}_{\mathrm{E}} \mathrm{X}-\mathrm{LA} \mathrm{T}_{\mathrm{E}} \mathrm{X}$ au profit d'une propriété de METAPOST qui permet de visualiser directement les figures, y compris les lettrages (composés avec une unique fonte, ce qui ne gêne en rien lorsque ces lettrages sont uniquement destinés à repérer des parties des figures). La méthode pour obtenir ces lettrages est exposée à la fin de ce chapitre. On conseille de procéder ainsi (au lecteur de personnaliser la méthode s'il le souhaite!) :

- Faire un répertoire de travail, mp par exemple.

- Créer un fichier figa.mp dans ce répertoire contenant un préambule (voir sect. 2.3.4) et le code pour toutes les figures du chapitre 1.

$\%$ figa.mp

... Preambule : affectations et definitions

$\backslash$ beginfig(1);

... code de la figure

lendfig;

METAPOST raconté aux piétons, chap. 1 
$\backslash$ beginfig(2);

... code de la figure

\endfig;

... etc.

end.

- Compiler avec la commande

mp figa

Ce traitement crée les fichiers PostScript des figures : figa.1, figa.2, etc. (cf. les arguments des macros beginfig). On peut intégrer ces figures dans un document $\mathrm{T}_{\mathrm{EX}} \mathrm{-L} \mathrm{T}_{\mathrm{E}} \mathrm{X}$ (ce qu'on ne fera pas pour le moment, comme annoncé ci-dessus). Pour ce faire, il est nécessaire de renommer les fichiers (par exemple : ren figa.1 figa1.eps, etc.) ou d'utiliser la déclaration \DeclaregraphicsRule $\{*\}\{$ eps $\}\{*\}\{*\}$ qui force \includegraphics à considérer les fichiers d'extension inconnue comme des fichiers .eps.

- Visualiser directement les figures figa.1, figa.2, etc. avec GSview : c'est l'idéal pour le débutant qui peut voir immédiatement la figure.

- On peut aussi transformer les fichiers de figure du format PostScript au format PDF avec les commandes :

epstopdf --out=figa1.pdf figa.1, etc.

La création de figures avec lettrages composés en $\mathrm{TE}_{\mathrm{E}} \mathrm{X}-\mathrm{L} \mathrm{T} \mathrm{T} \mathrm{X}$ sera exposée au chapitre 7. Maintenant, tout est prêt pour faire les premiers pas.

\section{COORDONNÉES CARTÉSIENNES}

Le point du plan de coordonnées $x=a$ bp et $y=b$ bp est représenté par la paire $(\mathrm{a}, \mathrm{b})$ puisque le bp (point PosTSCRIPT) est l'unité par défaut [19]. On peut aussi utiliser les unités reconnues par $\mathrm{TE}_{\mathrm{E}}[20]: \mathrm{cm}, \mathrm{mm}$, in, pt (point d'impression), etc. Par exemple, le point de coordonnées $x=8 \mathrm{~mm}$ et $y=5 \mathrm{~mm}$ est représenté par la paire $(8 \mathrm{~mm}, 5 \mathrm{~mm})$. On convient d'utiliser un facteur d'échelle u que l'on peut définir à l'aide des unités reconnues. Pour ce manuel, on prend $\mathrm{u}=1 \mathrm{~mm}$. Le point $x=8 \mathrm{~mm}$ et $y=5 \mathrm{~mm}$ est alors représenté par la paire $(8 \mathrm{u}, 5 \mathrm{u})$.

REMARQue. Lorsqu'une coordonnée est nulle, on note 0 (le bp est donc pris pour unité, ce qui n'a aucune importance). Par contre, une coordon-

Cahiers GUTenberg no 52-53-Octobre 2009 
née de valeur u doit être notée $1 u$ [20] (car 1u est en réalité une abréviation de $1 * u$ qui correspond à $1 \mathrm{u}$ mul en PostScript : l'omission du 1 déclencherait une erreur puisqu'il manquerait un élément dans la pile). Ce facteur d'échelle permet de réduire une figure [20] (en le diminuant) sans que cela affecte l'épaisseur des traits, le diamètre des points, etc. que l'on exprime en $\mathrm{pt}$ ).

Voici un exemple de tracé avec la commande draw [19] où l'on utilise les diverses représentations possibles des extrémités des segments. Pour simplifier, on place dans le préambule du fichier figa . mp les affectations nécessaires et l'appel du fichier macutil .mp (voir sect. 2.3.4) contenant quelques définitions très commodes, en particulier les définitions des commandes de tracé des axes et de la grille d'un pas de $1 \mathrm{~mm}$ (l'ancien papier millimétré !).

beginfig(1);

$\%<u=1 \mathrm{~mm}$;

axespapiermilli $(-1.5,-1.5,41.5,11.5)$

draw $(0,0)--(28.346,28.346)$;

draw $(15 \mathrm{u}, 10 \mathrm{u})--(25 \mathrm{u}, 0)$;

draw (1.1811in, 0$)--(4 \mathrm{~cm}, 28.453 \mathrm{pt})$

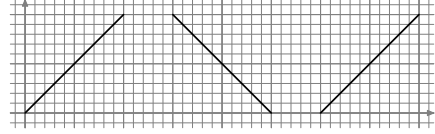

Figure 1

figa.mp

endfig;

Les caractères $\%<$ dans le code d'une figure signifient que ce qui suit sur la ligne est redondant, c'est-à-dire déjà défini plus haut (dans le préambule du fichier, dans le fichier macutil .mp ou dans le code d'une figure précédente), mais doit être utilisé pour traiter la figure seule.

Comme annoncé plus haut, on écrit un autre exemple en introduisant les variables du type paire $\mathrm{z} 1, \mathrm{z} 2$, etc., les variables du type chemin $\mathrm{p} 1$, $\mathrm{p} 2$, etc., et la transformation $\operatorname{shifted}(25 \mathrm{u}, 0)$ produisant une translation de l'élément précédent de $25 \mathrm{~mm}$ suivant les $x$ (et 0 suivant les $y$ ). beginfig(2);

axespapiermilli $(-1.5,-1.5,36.5,11.5)$

Figure 2

$\mathrm{z} 1=(0,0) ; \mathrm{z} 2=(10 \mathrm{u}, 0)$;

$z 3=(10 u, 10 u) ; z 4=(0,10 u)$;

path $\mathrm{p}[] ; \mathrm{p} 1=\mathrm{z} 4--\mathrm{z} 1--\mathrm{z} 2--\mathrm{z} 3 ; \mathrm{p} 2=\mathrm{p} 1--\mathrm{cycle}$;

draw $\mathrm{p} 1$;

figa.mp

draw p2 shifted $(25 \mathrm{u}, 0)$;

endfig;

On remarque que les variables paires $z$ [] (c'est-à-dire $z 1, z 2$, etc.) ne sont pas déclarées; c'est un cas spécial : elles sont prédéclarées ainsi 
que les variables du type nombre $\mathrm{x}[]$ et y []. Les variables chemin $\mathrm{p}$ [] sont ensuite déclarées; les crochets dans ces déclarations ont toujours le même sens : dans le cas de p par exemple, il s'agit d'une variable tableau à une dimension dont les éléments se nomment $\mathrm{p} 1, \mathrm{p} 2$, etc. On note encore que l'on a ajouté --cycle pour fermer le chemin et non pas --z4 qui n'est pas équivalent (ce choix est fondamental, en particulier pour des traits de forte épaisseur de chemins fermés destinés à être remplis).

\section{COORDONNÉES POLAIRES}

Il n'y a pas de représentation des points du plan prévue en coordonnées polaires; néanmoins, ce type de représentation est très utile, par exemple, pour définir une droite passant par un point donné et faisant un angle donné avec l'axe des $x$. On introduit dans l'exemple qui suit la macro polar pour représenter un point de coordonnées polaires $r, w$ par polar $(r, w)$ : les points $z 5$ et $z 6$ définissent bien une droite passant

Figure 3 figa.mp par le point $z 5$ et faisant un angle de $45^{\circ}$ avec l'axe des $x$. beginfig (3);

axespapiermilli $(-1.5,-1.5,31.5,13.5)$

$\%<$ def polar $(\operatorname{expr} r, w)=$

$\%<\quad(r * \cos d \mathrm{w}, r *$ sind $\mathrm{w})$ enddef;

$\mathrm{z} 1=\operatorname{polar}(12 \mathrm{u}, 0) ; \mathrm{z} 2=$ polar $(12 \mathrm{u}, 30)$;

$z 3=\operatorname{polar}(12 \mathrm{u}, 60) ; \mathrm{z} 4=$ polar $(12 \mathrm{u}, 90)$;

draw z1--z2--z3--z4;

$z 5=\operatorname{polar}(20 u, 0) ; z 6=z 5+\operatorname{polar}(15 u, 45)$;

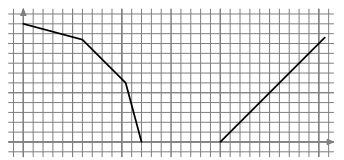

draw z5--z6;

endfig;

Dans l'exemple ci-dessus, la macro introduite est une macro à deux paramètres dont le principe est connu des utilisateurs de $\mathrm{T}_{\mathrm{E}} \mathrm{X}$; la syntaxe en est différente et plus exigeante. Tout cela sera approfondi plus loin.

\section{LETTRAGE DIRECT}

La commande [44] :

label.xxx ("aaa",zz);

compose la chaîne aaa au point de référence $z z$ et la déplace dans la direction $\mathrm{xxx}$ à la distance labeloffset (variable interne du type nombre dont la valeur par défaut est $3 \mathrm{bp}$ ); les valeurs possibles de la direction sont :

top, rt, bot, lft, urt, lrt, llft et ulft

Cahiers GUTenberg no 52-53-Octobre 2009 
Si aucune direction n'est donnée, la chaîne est centrée au point de référence.

Le choix de la fonte se fait en donnant des valeurs aux variables internes defaultfont (de type chaîne et de valeur par défaut cmr10) et defaultscale (de type nombre et de valeur par défaut 1). On reporte après la figure les explications détaillées du fonctionnement de METAPOST en ce qui concerne la composition de ces lettrages. Ces explications peuvent même être ignorées en première lecture. On reprend la deuxième figure en y rajoutant les commandes décrites ci-dessus. On écrit donc dans le préambule du fichier figa.mp (préambule répété pour les fichiers figb.mp, figc.mp et figd.mp et que l'on peut trouver à la section 2.3.4 accompagné de différentes explications) :

prologues : $=2$; labeloff set : $=1.7 \mathrm{pt}$; (lettrage plus proche)

defaultfont="phvr8r"; defaultscale: $=0.6$; (taille plus petite)

beginfig (4);

axespapiermilli $(-2.5,-3.5,38.5,13.5)$

Figure 4

$\mathrm{z} 1=(0,0) ; \mathrm{z} 2=(10 \mathrm{u}, 0) ; \mathrm{z} 3=(10 \mathrm{u}, 10 \mathrm{u}) ; \mathrm{z} 4=(0,10 \mathrm{u})$;

$\mathrm{z} 5=(5 \mathrm{u}, 5 \mathrm{u}) ;$ path $\mathrm{p}[] ; \mathrm{p} 1=\mathrm{z} 4--\mathrm{z} 1--\mathrm{z} 2--\mathrm{z} 3$;

p2=p1--cycle; draw p1;

label.rt ("z2", z2); label.lft ("z3" ,z3);

label.bot ("z1", z1); label.top ("z4", z4);

draw p2 shifted(25u,0);

label.lrt ("z2",z2 shifted $(25 \mathrm{u}, 0))$;

label.urt ("z3",z3 shifted $(25 \mathrm{u}, 0))$;

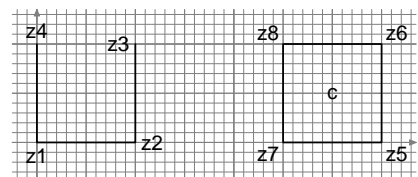

figa.mp

label.llft ("z1",z1 shifted $(25 \mathrm{u}, 0))$;

label.ulft ("z4",z4 shifted $(25 \mathrm{u}, 0))$;

label ("c",z5 shifted $(25 \mathrm{u}, 0))$;

endfig;

Pour terminer, voici les explications annoncées ci-dessus. Il faut que la fonte choisie ait un fichier métrique (fichier .tfm contenant toutes les dimensions des caractères) accessible à METAPOST; ensuite, si l'on veut voir directement la figure avec les lettrages, il faut que les fichiers contenant les dessins des lettres soient disponibles pour Gsview si on veut le résultat à l'écran et disponibles pour l'interpréteur PosTSCRIPT de l'imprimante si l'on veut le résultat sur le papier. On choisit par exemple la fonte phvr8r, dont le nom PostScRIPT est Helvetica. Cette fonte est une des 35 fontes disponibles sur les imprimantes dites PostSCRIPT et son . tfm se trouve sur toutes les installations $\mathrm{T}_{\mathrm{E}} \mathrm{X}-\mathrm{LAT} \mathrm{E} \mathrm{X}$ à jour. D'abord, 
METAPOST va lire le fichier phvr8r. tfm pour positionner les caractères du lettrage, ensuite, si l'on a donné la valeur 2 à la variable interne prologues, il va chercher dans l'installation $\mathrm{TE}_{\mathrm{E}} \mathrm{X}-\mathrm{LAT}_{\mathrm{E}} \mathrm{X}$ le fichier uhv . map dans lequel il va trouver le nom PostSCRIPT de la fonte choisie; on pourra éditer les fichiers des figures ayant un lettrage et vérifier qu'ils contiennent bien la ligne

/phvr8r Helvetica def Ainsi, gsview ou l'interpréteur PostSCRIPT de l'imprimante disposent du nom PosTSCRIPT de la fonte pour montrer ou imprimer les caractères.

Cahiers GUTenberg no 52-53-Octobre 2009 


\section{CHAPITRE 2 \\ Tracé de lignes brisées}

Dans ce chapitre, on trace des lignes brisées, ouvertes ou fermées, avec toutes les options possibles de tracé : épaisseur du trait, couleur du trait, forme de la jonction des segments élémentaires, etc. Ce choix est motivé par le désir de simplifier l'exposé et de permettre au lecteur d'utiliser le plus tôt possible la richesse contenue dans METAPOST. La plupart de ces options se retrouveront pour les lignes courbes. Bien sûr, on va continuer à exploiter toutes les propriétés du langage en écrivant quelques macros.

\section{SyNTAXe De BaSe dU TRACÉ}

La syntaxe pour la définition d'une ligne brisée passant par la suite de points $z 1, z 2, \ldots, z n$, est :

$\mathrm{p}=\mathrm{z1}--\mathrm{z2}--$ etc--zn; (ligne ouverte) [19], $\mathrm{p}=\mathrm{z1} 1--\mathrm{z} 2--$ etc--zn--cycle; (ligne fermée) [70].

Il y a ensuite les commandes utilisant ce chemin : draw p options; pour tracer le chemin [51], undraw p options; pour effacer le chemin [70], et, si le chemin est fermé :

fill p options; pour remplir le chemin [51], unfill p options: pour effacer l'intérieur du chemin [54], clip currentfile to $p$; pour découper et ne conserver que la partie de la figure courante intérieure au chemin $\mathrm{p}$ [75].

où options représente d'éventuelles options qui sont détaillées à la section suivante. On évitera, en débutant, l'utilisation de filldraw et unfilldraw plus délicates à utiliser. Le format metafun (distribution de $\mathrm{ConT}_{\mathrm{E}} \mathrm{XT}$ ) permet d'ombrer l'intérieur d'un chemin [172].

Sur l'exemple suivant (fichier de figures figb.mp) on trouve dans l'ordre six tracés : 
— un carré tracé, un carré rempli, un carré tracé et rempli ;

- un carré d'abord rempli puis tracé, ensuite le même carré d'abord tracé puis rempli afin de montrer la conséquence de l'inversion des opérations (nettement visible avec une forte épaisseur de trait) ;

- une utilisation astucieuse de la commande undraw pour tracer un trait double et une utilisation de la commande unfill pour gommer un petit carré déjà coloré afin, par exemple, de placer un lettrage.

Les commandes undraw et unfill correspondent à un effacement et donc ne sont pas équivalentes aux commandes fill et draw avec la couleur blanche.

On introduit dans l'exercice suivant la macro $c l$, reportée dans macutil.mp et détaillée à la section 2.2.5; son argument est l'intensité

Figure 1

figb.mp du gris (entre 0 et 1 ).

beginfig(1);

axespapiermilli $(-1.5,-1.5,42.5,26.5)$

$\%<$ def $\mathrm{cl}$ (expr $\mathrm{s})=$ withcolor $(\mathrm{s}, \mathrm{s}, \mathrm{s})$ enddef;

$z 1=(0,0) ; z 2=(10 u, 0) ; z 3=(10 u, 10 u)$;

$z 4=(0,10 u) ;$ path $p ; p=z 1--z 2--z 3--z 4--$ cycle;

draw $p$ shifted $(0,15 \mathrm{u})$;

fill p shifted $(15 \mathrm{u}, 15 \mathrm{u}) \mathrm{cl}(0.7)$;

fill p shifted(30u,15u) cl(0.7);

draw $\mathrm{p}$ shifted $(30 \mathrm{u}, 15 \mathrm{u})$;

fill $p$ shifted $(0,0) \mathrm{cl}(0.7)$

draw $\mathrm{p}$ shifted $(0,0)$

withpen pencircle scaled2mm;

draw $\mathrm{p}$ shifted $(15 \mathrm{u}, 0)$

withpen pencircle scaled2mm;

fill $\mathrm{p}$ shifted $(15 \mathrm{u}, 0) \mathrm{cl}(0.7)$;

fill p shifted $(30 u, 0)$ cl $(0.7)$;

draw $\mathrm{p}$ shifted(30u,0)withpen pencircle scaled3pt;

undraw $\mathrm{p}$ shifted $(30 \mathrm{u}, 0)$ withpen pencircle scaled1pt;

unfill p scaled 0.3 shifted $(32 u, 2 u)$;

endfig;

L'exemple suivant utilise la commande clip qui détoure ou découpe la partie de la figure faite avant cette commande et intérieure à un contour donné. La figure déjà faite est, à tout instant, la valeur de la variable interne currentpicture. Pour conserver cette figure initiale avant de la réduire par découpe, on la sauvegarde dans la variable du type dessin nommée dessininitial préalablement déclarée; on fait

Cahiers GUTenberg no 52-53-Octobre 2009 
la découpe en bas à droite; on sauvegarde la découpe dans une autre variable; on montre, en les déplaçant, la figure initiale (avec le chemin de découpe en traitillé) en haut et la copie de la découpe en bas à gauche. beginfig (2);

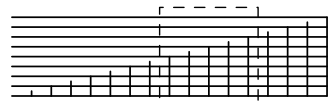

\section{Options DE TRACÉ}

On va passer en revue les options de tracé en donnant des exemples. La plupart de ces options restent valables pour les lignes courbes.

\section{1. ÉPAISSEUR DU TRAIT}

Cette épaisseur est choisie par l'option [70] :

withpen pencircle scaled $\mathrm{n}$ pt (n est un nombre) placée à la fin de la commande draw ou bien par la déclaration [20] :

pickup pencircle scaled $\mathrm{n}$ pt;

placée en avant de la commande draw concernée ou bien encore par la déclaration générale [70] :

drawoptions(withpen pencircle scaled n pt, option de couleur,...);

toujours placée avant la commande draw et qui peut inclure d'autres options que l'option d'épaisseur. Par défaut, cette épaisseur vaut $0.5 \mathrm{bp}$ (voir [19] pour la différence entre le pt et le bp; elle peut être bien souvent négligée).

Dans l'exemple suivant, on a testé d'abord l'option withpen. On a aussi testé la déclaration pickup et la commande de choix d'options drawoptions, et on a vérifié que l'on peut désactiver leur effet en appelant la plume par défaut, def aultpen, ou en donnant une liste d'options 
Figure 3

figb.mp

vide à drawoptions (ligne 1 et suivantes).

beginfig (3);

$\mathrm{z} 1=(0,0) ; \mathrm{z} 2=(10 \mathrm{u}, 0) ;$ path $\mathrm{p} ; \mathrm{p}=\mathrm{z} 1--\mathrm{z} 2$;

draw $\mathrm{p}$ shifted $(0,15 \mathrm{u})$ withpen pencircle scaled $4 \mathrm{pt} ; \%$ ligne 1

pickup pencircle scaled 2pt;draw p shifted(15u,15u);

pickup defaultpen; draw $\mathrm{p}$ shifted(30u,15u);

drawoptions (withpen pencircle scaled 4pt); $\quad \%$ ligne 2

draw $\mathrm{p}$ shifted $(0,10 \mathrm{u})$;

draw $\mathrm{p}$ shifted(15u,10u)withpen pencircle scaled 2pt;

drawoptions (); draw p shifted(30u,10u);

def $\operatorname{setlinewidth}(\operatorname{expr} \mathrm{s})=\quad \%$ ligne 3

pickup pencircle scaled $s$ enddef;

setlinewidth(4pt); draw $p$ shifted $(0,5 u)$;

draw $\mathrm{p}$ shifted $(15 \mathrm{u}, 5 \mathrm{u})$

withpen pencircle scaled $2 \mathrm{pt}$;

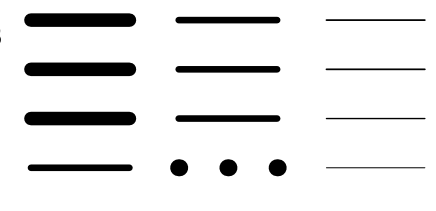

setlinewidth(0.5pt); draw $\mathrm{p} \operatorname{shifted}(30 \mathrm{u}, 5 \mathrm{u})$;

$\%<$ def $1 \mathrm{w}(\operatorname{expr} \mathrm{s})=$ withpen pencircle scaled $\mathrm{s}$ enddef;

draw $\mathrm{p} l \mathrm{lw}(2 \mathrm{pt})$;

$\%$ ligne 4

$\%<$ def drawpt (expr $\mathrm{z}, \mathrm{s})=$ draw $\mathrm{z}$ withpen pencircle scaled $\mathrm{s}$ enddef;

drawpt ((15u, 0), 5pt); drawpt ((20u, 0), 5pt); drawpt ((25u, 0), 5pt);

def $\operatorname{drawthin}(\operatorname{expr} \mathrm{p})=\mathrm{draw} \mathrm{p}$ withpen pencircle scaled . 3pt enddef;

drawthin $((30 u, 0)--(40 u, 0))$;

endfig;

On a aussi vérifié que, lorsque l'une de ces déclarations est active, on peut encore tracer un trait d'une épaisseur différente de celle fournie par cette déclaration grâce à l'option withpen placée en fin de la commande draw (ligne 2 et suivantes).

Ensuite, pour simplifier la saisie, on a défini la macro setlinewidth qui choisit l'épaisseur du trait, on l'a testée et on a aussi testé la macro Iw (reportée dans le fichier macutil.mp) abrégeant l'option withpen (ligne 3 jusqu'à ligne 4).

Enfin, puisqu'en traçant un segment de longueur nulle (commande draw avec une seule paire) on obtient un cercle plein (un point) dont le diamètre est l'épaisseur de trait choisie [21], on a introduit et testé la macro drawpt (reportée dans macutil.mp). Enfin, puisque dans une figure ou une suite de figures d'un même ouvrage, on n'aura qu'un très petit nombre de sortes de traits (pour des raisons de qualité pédagogique), on a tout intérêt à définir les quelques macros correspondantes pour alléger la saisie : par exemple la macro drawthin qui pourrait aussi contenir une

Cahiers GUTenberg no 52-53 - Octobre 2009 
option de couleur.

Remarque. Pour terminer cette sous-section, on explique le choix du vocabulaire de l'option withpen et de la déclaration pickup. METAFONT a été conçu pour travailler comme un humain qui trace des traits avec des feutres qu'il fait glisser sur le papier. Pour augmenter l'épaisseur du trait, il faut augmenter le diamètre de la pointe du feutre d'où scaled n pt. Mais le concepteur a aussi prévu l'utilisation de feutres dont la section de la pointe n'est pas forcément circulaire : ainsi, avec une plume elliptique de grand axe parallèle à l'axe des $x$, on conçoit aisément qu'un déplacement suivant l'axe des $y$ donne un trait plus épais qu'un déplacement suivant l'axe des $x$; cela permet le tracé de caractères calligraphiques... et se retrouve dans METAPOST [73].

\subsection{TERMINAISONS ET JONCTIONS DE TRAITS}

Les terminaisons et jonctions des traits sont choisies par l'intermédiaire de deux variables pouvant prendre trois valeurs.

Pour la terminaison, la variable et ses trois valeurs possibles sont [67] : linecap:=butt; ou squared; ou rounded; (défaut)

Pour la jonction, la variable et ses trois valeurs possibles sont [68] :

linejoin:=beveled; ou mitered; ou rounded; (défaut)

L'exemple suivant montre les six possibilités.

beginfig(4);

$\%<$ def $\mathrm{cl}(\operatorname{expr} \mathrm{s})=$ withcolor $(\mathrm{s}, \mathrm{s}, \mathrm{s})$ enddef;

Figure 4

figb.mp

path $\mathrm{p} ; \mathrm{p}=(0,0)--(10 \mathrm{u}, 0)$; drawoptions $\left(\mathrm{lw}_{\mathrm{w}}(2 \mathrm{~mm}) \mathrm{cl}(0.7)\right)$;

linecap:=butt; draw $\mathrm{p}$ shifted $(0 \mathrm{u}, 12 \mathrm{u})$;

linecap:=squared; draw $\mathrm{p} \operatorname{shifted}(15 \mathrm{u}, 12 \mathrm{u})$;

linecap: =rounded;

draw $\mathrm{p}$ shifted $(30 \mathrm{u}, 12 \mathrm{u})$;

path $\mathrm{p} ; \mathrm{p}=(0,0)--(5 \mathrm{u}, 7 \mathrm{u})--(10 \mathrm{u}, 0)$;

linejoin:=beveled; draw p;

linejoin:=mitered;

draw $\mathrm{p}$ shifted $(15 \mathrm{u}, 0)$; linejoin:=rounded;

draw $\mathrm{p}$ shifted $(30 \mathrm{u}, 0)$; drawoptions (1w $(2 \mathrm{pt}))$;

drawpt $((0,12 u), 2 p t) ; \operatorname{drawpt}((15 u, 12 u), 2 p t) ; \operatorname{drawpt}((30 u, 12 u), 2 p t)$;

drawpt ((10u, 12u), 2pt); drawpt ( (25u, 12u), 2pt) ; drawpt ((40u, 12u) , 2pt) ;

$\operatorname{drawpt}((5 \mathrm{u}, 7 \mathrm{u}), 2 \mathrm{pt}) ; \operatorname{drawpt}((20 \mathrm{u}, 7 \mathrm{u}), 2 \mathrm{pt}) ; \operatorname{drawpt}((35 \mathrm{u}, 7 \mathrm{u}), 2 \mathrm{pt})$;

endfig;

On constate que les deux dernières possibilités de terminaisons conduisent à un dépassement aux extrémités (représentées par un point 
noir sur le figure).

Après un changement provisoire, il ne faut pas oublier de reprendre la valeur par défaut. Il y a une autre possibilité pour un tel changement provisoire [78]; elle n'abrège pas la saisie et donc on n'en dira pas davantage. Sur l'exemple précédent l'ordre de présentation assure le retour aux valeurs par défaut.

\subsection{Pointillés et traitillés}

Les pointillés sont obtenus avec l'option [64] :

dashed withdots scaled $\mathrm{n}$

placée en fin de la commande draw (n est un nombre).

Les traitillés s'obtiennent avec l'option [64] :

dashed evenly scaled $\mathrm{n}$

aussi placée en fin de la commande draw (n est encore un nombre dont on va tester l'action sur l'exemple suivant).

L'exemple suivant donne trois lignes de pointillés suivies de trois

Figure 5 lignes de traitillés avec différents espacements.

figb.mp

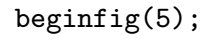

Dans certains cas, on peut être amené à rechercher la perfection : on voit sur l'exemple que les traitillés commencent par un tiret; à l'autre extrémité, il peut y avoir un blanc ou un tiret coupé; pour assurer une certaine symétrie, on peut déplacer le début des pointillés ou des traitillés en ajoutant à l'option shifted $(\mathrm{d}, 0)$ où $d$ est une distance 
choisie par tâtonnement. La septième ligne reprend la sixième avec un décalage de 2,4 pt assurant la symétrie. L'exemple montre aussi comment on peut définir un traitillé de style personnel avec la définition d'un motif spécifique, par exemple [65] :

dashpattern(on $6 \mathrm{pt}$ off $3 \mathrm{pt}$ on $3 \mathrm{pt}$ off $3 \mathrm{pt}$ )

qui donne, en se répétant indéfiniment, un traitillé dont le tiret de base a une longueur alternativement de 3 pt et de 6 pt (ligne 8). Enfin, on propose et teste une macro donnant un traitillé afin d'abréger la saisie (ligne 9).

\subsection{FLÈCHES}

La construction des flèches par défaut est assez pauvre : on dispose d'un seul type de flèche et de trois commandes [69] :

drawarrow chemin options;

qui place une flèche à l'extrémité du chemin, drawarrow reverse chemin options;

qui place une flèche inversée à l'origine du chemin et drawdblarrow chemin options;

qui place une flèche à chaque extrémité du chemin. L'exemple suivant montre différentes possibilités.

beginfig(6);

linejoin:=mitered; path $\mathrm{p} ; \mathrm{p}=(0,0)--(15 \mathrm{u}, 0)$;

Figure 6

drawarrow $\mathrm{p}$ shifted $(0,12 \mathrm{u}) \mathrm{lw}(0.2 \mathrm{pt})$;

drawarrow $\mathrm{p}$ shifted $(20 \mathrm{u}, 12 \mathrm{u})$;

drawarrow p shifted $(0,8 \mathrm{u})$ lw(1pt);

drawarrow p shifted(20u,8u) Iw(2pt);

drawarrow reverse $p$ shifted $(0,4 u)$;

drawdblarrow $\mathrm{p}$ shifted $(20 \mathrm{u}, 4 \mathrm{u})$;

drawarrow p lw(8pt) cl(0.5); \% fleche

draw p lw(8pt) cl(0.75); $\%$ trait

linecap:=butt;

drawarrow $\mathrm{p}$ shifted $(20 \mathrm{u}, 0) \mathrm{lw}(8 \mathrm{pt}) \mathrm{cl}(0.5) ; \%$ fleche

draw $\mathrm{p}$ shifted $(20 \mathrm{u}, 0) \mathrm{lw}(8 \mathrm{pt}) \mathrm{cl}(0.75) ; \quad \%$ trait

linecap:=rounded; linejoin:=rounded; draw $(15 \mathrm{u},-2 \mathrm{u})--(15 \mathrm{u}, 14 \mathrm{u})$

endfig;

$1 w(0.2 p t) ; \operatorname{draw}(35 \mathrm{u},-2 \mathrm{u})--(35 \mathrm{u}, 14 \mathrm{u}) \mathrm{lw}(0.2 \mathrm{pt})$;

Les flèches sont caractérisées par deux variables internes prédéfinies [69] : ahlength et ahangle. La première a dû être fortement diminuée. Sur l'exemple précédent, on trouve un test des trois commandes de tracé 
de flèches avec linejoin:=mitered; pour avoir des flèches bien pointues. Par construction même [69], la flèche dépasse l'extrémité du chemin; cette propriété est bien visible pour les chemins de forte épaisseur et est clairement montrée par les deux dernières flèches de l'exemple cidessus où la flèche entière est tracée en gris foncé puis surchargée par le chemin (un segment dans ce cas) en gris clair. Cette construction a aussi pour conséquence que la valeur butt de la variable linecap entraîne le même effet que la valeur beveled de la variable linejoin comme cela se découvre sur la flèche en bas à droite.

\subsection{Couleurs}

La couleur est gérée dans METAPOST suivant le codage RGB. Une couleur est représentée par un triplet de trois nombres inférieurs à 1 . Les couleurs prédéfinies sont red, green, blue, white et black (défaut) [34]; on peut définir toutes les couleurs que l'on veut, par exemple yelow $=(1,1,0)$ définit le jaune. Les tracés et les remplissages sont colorés en utilisant l'option [51] :

withcolor couleur

où couleur est le nom d'une couleur ou tout simplement un triplet

Figure 7

figb.mp définissant une couleur.

beginfig( 7 );

axespapiermilli $(-2.5,-2.5,37.5,14.5)$; path $\mathrm{p} ; \mathrm{p}=(0,0)--(15 \mathrm{u}, 0)$;

color yellow, magenta, cyan; yellow $(1,1,0)$; magenta $=(1,0,1)$;

cyan $=(0,1,1)$; drawoptions (withpen pencircle scaled $2 \mathrm{~mm}$ );

draw $\mathrm{p}$ shifted $(\mathrm{Ou}, 12 \mathrm{u})$ withcolor white;

draw $p$ shifted $(20 u, 12 u)$ withcolor red;

draw $p$ shifted $(0,8 u)$ withcolor green;

draw $\mathrm{p}$ shifted $(20 \mathrm{u}, 8 \mathrm{u})$ withcolor blue;

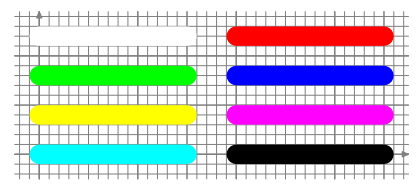

draw $\mathrm{p}$ shifted $(0,4 \mathrm{u})$ withcolor $(1,1,0)$;

draw $\mathrm{p}$ shifted(20u,4u) withcolor magenta;

draw $\mathrm{p}$ withcolor cyan; draw $\mathrm{p}$ shifted(20u,0) withcolor black; endfig;

L'exemple ci-dessus montre l'utilisation de cette option de couleur dans les tracés mais elle s'utilise de la même manière pour les remplissages.

On peut additionner, soustraire et multiplier par un nombre ces triplets : le résultat est automatiquement traité par METAPOST pour que les trois nombres soient inférieurs ou égaux à 1 .

Cahiers GUTenberg no 52-53-Octobre 2009 


\section{Compléments}

On trouvera dans certains chapitres une section constituée par des aspects de METAPOST en relation plus ou moins directe avec l'objet du chapitre; on y trouvera parfois quelques précisions concernant la syntaxe.

\subsection{RÉSOLUTION D'ÉQUATIONS LINÉAIRES}

Puisque ce chapitre est consacré aux lignes brisées constituées par des segments de droite, il est naturel de présenter la résolution des systèmes d'équations linéaires par METAPOST, résolution qui permet en particulier de déterminer, dans la sous-section suivante, le point d'intersection de deux droites (quand il existe, bien entendu!).

On a vu que l'on représente un point du plan par une variable du type paire; si l'on a une paire $z 1$, alors on a les relations [40] :

$\mathrm{x} 1=x$ part $\mathrm{z} 1$; et $\mathrm{y} 1=y$ part $\mathrm{z} 1$;

qui donnent $\mathrm{x} 1$ et $\mathrm{y} 1(\mathrm{z} 1=(\mathrm{x} 1, \mathrm{y} 1))$. Cela est particulièrement utile lorsque $\mathrm{z} 1$ est le résultat d'un calcul, ces deux opérateurs xpart et ypart permettent alors de déterminer l'un ou l'autre des éléments de la paire $z 1$. Voici un exemple où l'on veut utiliser uniquement l'abscisse $\mathrm{x} 1 \mathrm{de}$ $z 1$.

beginfig(8);

axespapiermilli $(-1.5,-1.5,36.5,16.5)$

$z 1+z 2=(5 u, 2 u) ; z 1-z 2=(2 u, 5 u) ; a=x p a r t z 1$;

draw $(35 \mathrm{u}, 0)--(\mathrm{a}, 15 \mathrm{u})$; drawpt (z1,3pt);

label.urt (" $(\mathrm{a}, 15 \mathrm{u})$ ", $(\mathrm{a}, 15 \mathrm{u}))$;

endfig;

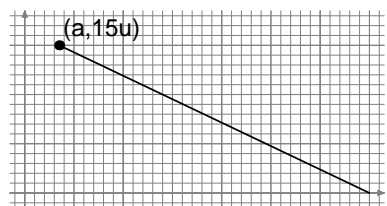

Figure 8

figb.mp

\subsection{INTERSECTION DE DROITES}

Une droite est définie par deux points, $z 1$ et z2 par exemple. Alors tout point de cette droite peut s'écrire sous la forme $z_{5}=z_{1}+a \cdot\left(z_{2}-z_{1}\right)$, ce que l'on note [30], [M 9] :

$\mathrm{z} 5=\mathrm{a}[\mathrm{z} 1, \mathrm{z} 2]$;

( $a=0.5$ correspond au milieu du segment d'origine $z 1$ et d'extrémité $z 2$ ). Si l'on a une autre droite définie par z3 et $z 4$, tout point de cette droite est encore de la forme :

z5 $=\mathrm{b}[\mathrm{z} 3, \mathrm{z} 4]$;

Ces deux équations sont en fait quatre équations à quatre inconnues x5, 
$\mathrm{y} 5$, a et b que METAPOST résout; en particulier z5 est disponible pour

Figure 9

figb.mp un tracé, comme le montre l'exemple suivant :

beginfig (9);

axespapiermilli $(-1.5,-1.5,31.5,21.5)$;

$\mathrm{z} 1=(0,0) ; \mathrm{z} 2=(10 \mathrm{u}, 10 \mathrm{u}) ; \mathrm{z} 3=(30 \mathrm{u}, 0) ; \mathrm{z} 4=(25 \mathrm{u}, 10 \mathrm{u})$

numeric $a ; b ; z 5=a[z 1, z 2]=b[z 3, z 4]$;

draw $z 1--z 5$; draw $z 3--z 5$; drawpt (z1,2pt);

drawpt (z2,2pt); drawpt (z3,2pt); drawpt (z4,2pt);

drawpt (z5,3pt); label.bot ("z1", z1);

label.ulft ("z2" ,z2); label.bot ("z3", z3);

label.urt ("z4" ,z4); label.top("z5" ,z5);

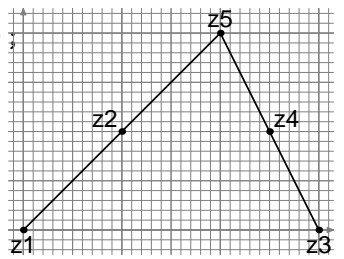

endfig;

Ici on est dans un cas où il faut déclarer les variables du type nombre (elles ne sont pas définies en les égalant à un nombre : ce sont des inconnues!). Pour ces variables annexes dont la valeur est sans intérêt, on dispose d'un unique mot whatever qui, de façon transparente, définit une nouvelle variable du type nombre chaque fois qu'il est utilisé : les équations ci-dessus s'écrivent ainsi :

$z 5=$ whatever $[z 1, z 2]=$ whatever $[z 3, z 4]$;

\subsection{PRÉCisions De CERTAins POINTS DE SyNTAXe}

On a utilisé cinq types de variables qui doivent être (presque toujours) déclarées :

$a, b, x[], y[]$ dans $b=1 ; b=3 ; x 1=5 ; y 2=7$; etc., type nombre sans déclaration;

numeric $c, s[]$ dans $z 3=c[z 2, z 1] ; s 1=9 ; s 2=8$; etc., type nombre; $z$ [] dans : $z 1=(7,9)$; etc., type paire sans déclaration;

pair qa, $\mathrm{q}[]$; dans qa $=(7,8) ; \mathrm{q} 1=(3,4) ; \mathrm{q} 2(5,6)$; etc., type paire;

color yellow; dans yellow $=(1,1,0)$; type couleur;

path $\mathrm{pa}, \mathrm{p}[] ;$ dans $\mathrm{pa}=\mathrm{z} 1--\mathrm{z} 4 ; \mathrm{p} 1=\mathrm{z} 1--\mathrm{z} 2 ; \mathrm{p} 2=\mathrm{z} 3--\mathrm{z} 4$; etc., type chemin;

picture dessin; dans dessin=currentpisture; type dessin.

Toutes les variables tableaux de tous les types doivent être déclarées sauf: $x[], y[]$ et $z$ [] qui sont prédéclarées (nombres et paire); en outre xi et yi sont, par le biais d'une macro, respectivement les éléments de la paire $z i$ (par exemple, $x 1=1$; et $z 1=(2,3)$; sont incompatibles); les valeurs de ces variables $(x[], y[]$ et $z[])$ sont effacées par les macros beginfig.

Cahiers GUTenberg no 52-53-Octobre 2009 
Quand une nouvelle variable est introduite, elle est considérée du type numérique; si on écrit a $=1$; sans déclaration, il n'y a pas de message d'erreur car 1 est bien un nombre. Par contre, si l'on écrit $q=(3,4)$; sans déclarer q comme variable du type paire, alors on a un message du type : «inconsistent equation (numeric=pair)» pour rappeler qu'il manque la déclaration. En fait, il n'y a que les variables nombre qui ne nécessitent pas de déclaration si elles sont introduites par une équation non ambiguë du point de vue syntaxique, ce qui n'est pas le cas de l'équation $z 3=c[z 2, z 1]$ qui exige une déclaration préalable de c (voir la deuxième ligne de la liste ci-dessus). On a rencontré aussi les types de variables transformation (exemple: shifted) et plume (exemple : pencircle); on y reviendra plus loin car il en manque encore deux et il y a aussi les variables internes.

On a aussi défini des macros; la syntaxe des ces définitions est [76] : def nom-macro = texte de remplacement enddef; pour une macro sans paramètre :

def nom-macro(expr $s, t, e t c.)=$ texte de remplacement incluant les paramètres s,t,etc. enddef; pour une macro avec paramètres; expr signifie que les paramètres qui suivent sont des variables des neuf types qui peuvent être définis par METAPOST (dont trois n'ont pas encore été rencontrés). Une définition plus générale est [83] :

def nom-macro(expr s,t,etc.) (text $t \mathrm{t})=$

texte de rempl. incl. les param. $s, t, e t c$. et tt enddf; où text signifie que le paramètre qui suit est du texte, par exemple une suite d'instructions (cas de la macro axespapiermilli dans macutil.mp); ce type de paramètre apparaîtra aussi dans d'autres cas (cas de la macro arcd à la section 3.5.1). Il faut introduire autant de groupes (text ...) qu'il y a de paramètres du type text car la virgule ne joue plus le rôle de séparateur (elle peut faire partie des paramètres).

\subsection{Préambule du fichier des Figures}

On écrit les affectations et les définitions utilisées par les figures dans les préambules des fichiers des figures.

Si une affectation est répétée; elle ne donne pas lieu à une erreur mais simplement à un avertissement de redondance. On pourra vérifier en enlevant les caractères $\%<$ devant la ligne $u=1 \mathrm{~mm}$; de la figure 1 (chap. 1) 
et devant la ligne $p=z 1--z 2--z 3--z 4--c y c l e$; de la figure 2 (chap. 2).

Les déclarations de variables et les définitions de macros peuvent ne pas être répétées, elles restent valables pour les figures suivantes. $\mathrm{Si}$ elles sont répétées, elles ne donnent pas lieu à message. Il vaut mieux répéter systématiquement les déclarations pour éviter des erreurs : si une figure contient $\mathrm{a}=1$; et la suivante $\mathrm{a}=2$; alors un message signale l'incompatibilité : il faut rajouter numeric a; dans la deuxième figure, la déclaration effaçant la précédente égalité.

Voici le préambule des fichiers figa.mp, ..., figd.mp des figures des chapitres 1 à 4 . Les définitions souvent utilisées y sont rassemblées.

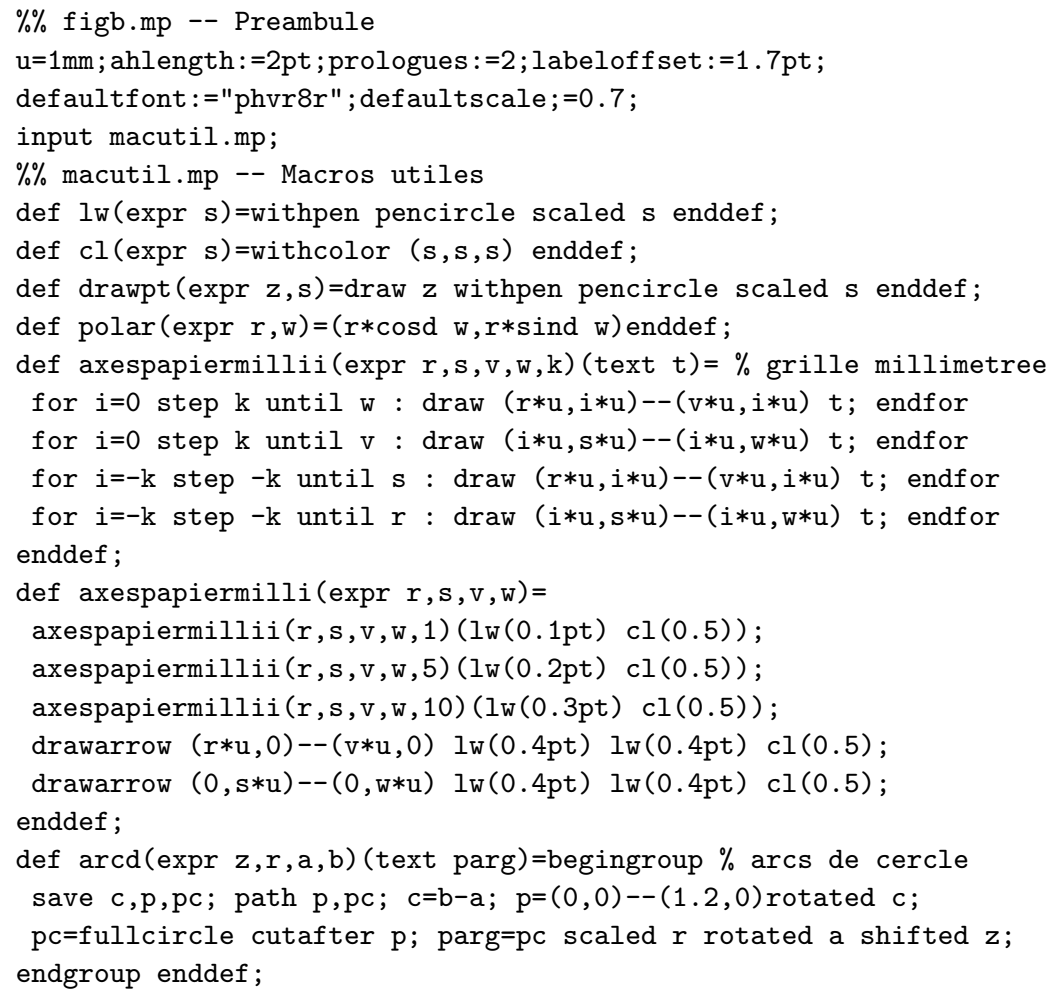

Cahiers GUTenberg no 52-53-Octobre 2009 


\section{CHAPITRE 3 \\ Tracé de lignes courbes}

Dans ce chapitre, on va découvrir la puissance de METAPOST (héritée bien entendu de la puissance de METAFONT comme cela est expliqué dans l'introduction). On va pouvoir tracer des courbes passant par des points donnés, appelés points guides, avec continuité de la tangente en ces points. Ensuite, on va pouvoir intervenir sur la forme de ces courbes par le biais d'options faciles à utiliser (par exemple, on va pouvoir imposer la direction de la tangente en chaque point guide, modifier la «rondeur» des éléments des courbes situés entre deux points guides, etc.). On donne d'abord un aperçu très partiel de ce que l'on peut faire en traçant des variantes d'une courbe passant par cinq points guides. Ensuite, on expose la méthode de tracé utilisée par METAPOST (courbes de Bézier cubiques). Enfin, on fait le tour des possibilités offertes pour modifier la forme de ces courbes afin de leur donner les propriétés voulues. On termine le chapitre en exposant les puissantes commandes disponibles (par exemple, pour découper des morceaux de courbe, pour déterminer les points d'intersection des courbes, pour tracer des tangentes aux courbes, etc.). Dans ce chapitre, le code des lettrages n'est pas reporté dans le listing des exercices.

\section{UN TRACÉ ÉLÉMENTAIRE POUR DÉBUTER}

On considère cinq points représentés par $z 0, z 1, z 2, z 3$ et $z 4$ (fichier de figures figc.mp). On trace plusieurs courbes passant par ces cinq points appelés points guides. La syntaxe ne diffère de la syntaxe de tracé des lignes brisées que par le remplacement de -- par . . ; sur l'exemple, page suivante, il y a d'abord la courbe ouverte allant de z0 à z4, ensuite on ferme cette courbe en revenant au point $z 0$ et enfin on ferme la courbe avec . cycle au lieu de revenir en $\mathrm{z} 0$ : cette option ferme la courbe et assure la continuité de la tangente [22]. 
Figure 1

figc.mp
Figure 2 figc.mp beginfig(1);

path $\mathrm{p}[] ; \mathrm{z} 0=(2 \mathrm{u}, 0) ; \mathrm{z} 1=(14 \mathrm{u}, 13 \mathrm{u})$;

$z 2=(9 u, 30 u) ; z 3=(2 u, 23 u) ; z 4=(5 u, 16 u)$;

draw $z 0 \ldots z 1 \ldots z 2 \ldots z 3 . . z 4$;

for $i=0$ upto $4: \operatorname{drawpt}(z[i], 2 p t)$; endfor $\mathrm{p} 1=\mathrm{z} 0 \ldots \mathrm{z} 1 \ldots \mathrm{z} 2 \ldots \mathrm{z} 3 \ldots \mathrm{z} 4 \ldots \mathrm{z} 0$;

draw p1 shifted(15u,0);

for $i=0$ upto $4: \operatorname{drawpt}(z[i], 2 p t)$

shifted $(15 \mathrm{u}, 0)$; endfor

p2=z0 . z1 . z2 . .z3 . z4 . cycle;

draw p2 shifted(30u,0);

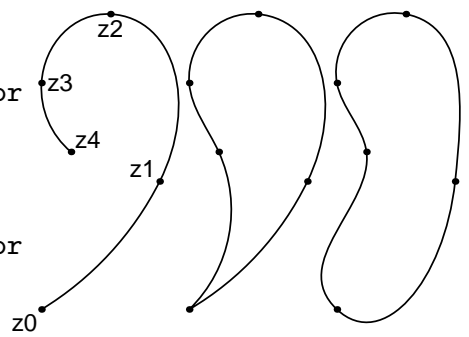

for $i=0$ upto $4: \operatorname{drawpt}(z[i], 2 p t)$ shifted $(30 u, 0)$; endfor endfig;

L'exemple ci-dessus montre bien l'importance de l'utilisation de cycle pour fermer les lignes courbes (on reviendra sur ce point par la suite). Pour l'exemple suivant, on reprend la courbe fermée correctement puis on remplace les deux derniers éléments par deux segments ( . . remplacé deux fois par --) : on constate qu'il n'y a pas continuité de la tangente en $z 0$ et z3, ainsi qu'en z4 où se joignent les deux segments; on montre ensuite que l'on peut imposer la continuité de la tangente au point z3 (tangente dans le prolongement du premier segment) et que l'on peut imposer une direction à la tangente au point z2 (tangente orientée faisant un angle de $190^{\circ}$ avec l'axe des $x$ ) [24], [M 16].

beginfig (2);

$\%<$ def $l_{\mathrm{wcl}}=l_{\mathrm{w}}(0.4 \mathrm{pt}) \mathrm{cl}(0.4)$ enddef;

path $\mathrm{p}[] ; \mathrm{z} 0=(2 \mathrm{u}, 0) ; \mathrm{z} 1=(14 \mathrm{u}, 13 \mathrm{u}) ; \mathrm{z} 2=(9 \mathrm{u}, 30 \mathrm{u}) ; \mathrm{z} 3=(2 \mathrm{u}, 23 \mathrm{u})$;

$z 4=(5 u, 16 u) ; p 1=z 0 \ldots z 1 \ldots z 2 \ldots z 3 \ldots z 4 \ldots$ cycle; draw $p 1 ;$ for $i=0$ upto 4 :

drawpt (z[i],2pt); endfor

p2=z0 . z1 . z 2 . .z3--z4--cycle;

draw p2 shifted(15u,0);

for $i=0$ upto $4: \operatorname{drawpt}(z[i], 2 p t)$ shifted $(15 u, 0)$; endfor

$\mathrm{p}[4]=\mathrm{z} 3--1.8[\mathrm{z} 4, \mathrm{z} 3]$;

draw p4 shifted(30u,0) 1wcl;

$\mathrm{p} 5=\mathrm{z} 2+\mathrm{pol} \operatorname{ar}(7 \mathrm{u}, 190)--$

z2--polar $(7 \mathrm{u}, 190)$;

draw p5 shifted $(30 \mathrm{u}, 0) 1 \mathrm{wcl}$;

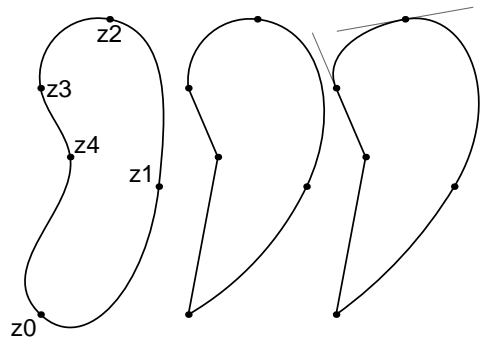

p3=z0 . z1 . . dir190\}z2 . . \{z4-z3\}z3--z4--cycle;

draw p3 shifted $(30 \mathrm{u}, 0)$;

for $i=0$ upto $4: \operatorname{drawpt}(z[i], 2 p t) \operatorname{shifted}(30 u, 0)$; endfor endfig;

Cahiers GUTenberg no 52-53 - Octobre 2009 
Tout cela paraît un peu mystérieux; pour comprendre, il faut examiner la méthode de tracé : il faut se familiariser avec les courbes de Bézier cubiques.

\section{Courbes de BÉZIER CUBIQUeS}

Considérons quatre points $z 1, z c 1, z c 2$ et $z 2$; ces quatre points définissent de façon unique une courbe dite de Bézier dont l'origine est $z 1$, l'extrémité est $z 2$ (elle est donc orientée de z1 vers z2, cela est fondamental pour la suite) et les points de contrôle sont $\mathrm{zc} 1$ et $\mathrm{zc} 2$ : ce sont seulement ces deux points qui caractérisent la forme de la courbe entre son origine et son extrémité. Du point de vue mathématique, cette courbe est définie paramétriquement par un polynôme du troisième degré d'un type particulier : $Z(t)$ pour $t \in[0,1]$ [23], [M 14]. La syntaxe pour tracer la courbe est [24] :

draw z1..controls zc1 and zc2..z2;

Ce tracé fait l'objet de l'exemple suivant. La caractéristique première de cette courbe est que la tangente orientée au point $z 1$ est dans la direction de zc1 et que la tangente orientée en $z 2$ est dans la direction opposée à la direction de zc2. La deuxième caractéristique est que les points de contrôle donnent l'impression de «tirer " la courbe vers eux comme le montre l'exemple où les point de contrôle sont éloignés (suivant les flèches) de manière à conserver les directions des tangentes aux deux extrémités. Ces courbes de Bézier peuvent être construites point par point par un méthode géométrique [M 13].

beginfig (3);

$z 1=(0,0) ; z 2=(40 u, 0) ; \operatorname{drawpt}(z 1,3 p t) ; \operatorname{drawpt}(z 2,3 p t) ;$

Figure 3

drawarrow z1--polar (42u, 45) Iwcl; drawarrow z2--(25u, 30u) lwcl;

pair $\mathrm{zc}[] ; \mathrm{zc} 1=(10 \mathrm{u}, 10 \mathrm{u}) ; \mathrm{zc} 2=(36 \mathrm{u}, 8 \mathrm{u})$;

drawpt (zc1,2pt); drawpt (zc2, 2pt);

draw $z 1 \ldots$ controls $\mathrm{zc} 1$ and $\mathrm{zc} 2 . . \mathrm{z} 2$;

$\mathrm{zc} 3=(16 \mathrm{u}, 16 \mathrm{u}) ; \mathrm{zc} 4=(32 \mathrm{u}, 16 \mathrm{u})$;

drawpt (zc3,1.2pt); drawpt (zc4,1.2pt);

draw z1..controls $\mathrm{zc} 3$ and $\mathrm{zc} 4 . . \mathrm{z} 2$ $1 \mathrm{w}(0.35 \mathrm{pt})$

$\mathrm{zc} 5=(22 \mathrm{u}, 22 \mathrm{u}) ; \mathrm{zc} 6=(30 \mathrm{u}, 20 \mathrm{u})$;

drawpt (zc5,1.2pt); drawpt (zc6,1.2pt);

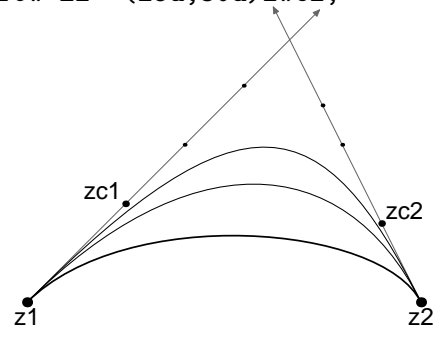

draw z1..controls zc5 and zc6..z2 lw(0.35pt);

endfig;

METAPOST raconté aux piétons, chap. 3 
Figure 4

figc.mp

On peut aussi obtenir un quart de cercle avec une très grande précision par la courbe de Bézier de l'exemple suivant :
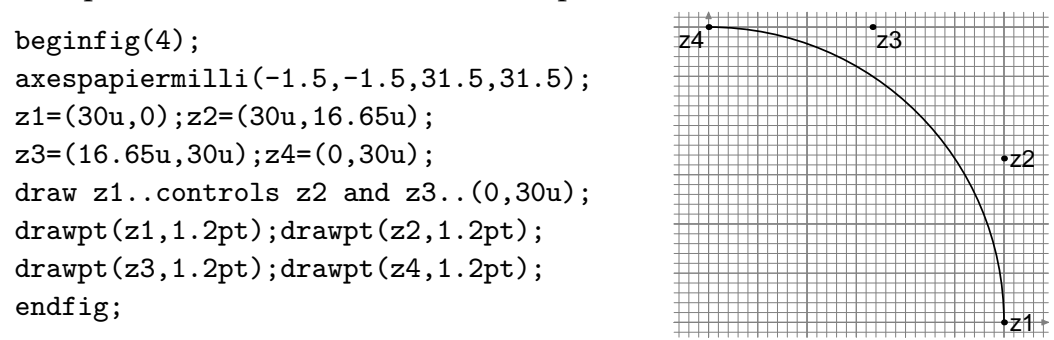

Pour les courbes de la section précédente, on n’a pas disposé de points de contrôle pour les différents éléments constitutifs et pourtant, ces courbes sont bien "sympathiques». On va maintenant voir les différentes possibilités de METAPOST pour construire des courbes, possibilités basées sur les courbes de Bézier cubiques.

\section{DifFÉRENTES POSSIBILITÉS DE TRACÉS DE COURBES}

\subsection{TRACÉ AVEC LeS SEULS POINTS GUIDES}

A la section 3.1, on a construit des courbes en ne donnant que les points par lesquels elles doivent passer. METAPOST trace ces courbes en construisant $n-1$ (resp. $n$ ) courbes de Bézier pour les courbes ouvertes (resp. fermées) définies par $n$ points guides sans la donnée de points de contrôle. Le programme détermine, par un algorithme complexe qui sort du cadre de ce manuel et des références citées, les points de contrôle nécessaires pour obtenir la courbe la «meilleure " possible. Evidemment ce qualificatif est subjectif!

\subsection{TRACÉ EN FiXANT LeS TANGENTES AUX POINTS GUIDES}

On peut tout de même donner un certain sens à ce qualificatif: on peut imaginer que l'application pour laquelle on dessine la courbe exige que les tangentes orientées à la courbe aux points guides aient des directions données. On a déjà vu que cela est possible à la fin de la section 7 . On précise maintenant les deux formes de syntaxe de la commande qui permet de satisfaire à cette exigence [24], [M 16] :

draw $z 1\{$ dir nombre\}..z2\{zi-zj\}..z3\{...\}.. ..\{...\}z4; où nombre est la valeur souhaitée de l'angle en degrés que la tangente orientée doit faire avec l'axe des $x$ croissants, et où zi-zj désigne un 
vecteur d'origine $z j$ et d'extrémité $z i$ dont on veut que la tangente orientée ait la direction.

On montre sur l'exemple suivant des tracés qui appellent quelques commentaires. On donne les trois points $z 1=(0,0), z 2=(20 u, 20 u)$ et $z 3=(40 u, 0)$; en donnant les bonnes directions aux tangentes en ces points, on obtient, ligne A, presque le demi-cercle obtenu avec la courbe de Bézier et les points de contrôle particuliers déjà utilisé à la section précédente, ligne $\mathrm{B}$, pour tracer le quart de cercle; plus suprenant, la considération des points extrêmes seulement et des directions des tangentes en ces points donne encore, ligne $\mathrm{C}$, presque le demi-cercle; plus surprenant encore, la seule donnée de deux points points et de la commande de fermeture du chemin (. . cycle) produit pratiquement un cercle, ligne E. On peut dire que l'algorithme de détermination des points de contrôle tend à faire un chemin le plus « arrondi " possible.

beginfig (5);

$\mathrm{u}:=0.97 \mathrm{~mm} ; \mathrm{z} 1=(0,0) ; \mathrm{z} 2=(20 \mathrm{u}, 20 \mathrm{u}) ; \mathrm{z} 3=(40 \mathrm{u}, 0)$;

Figure 5

drawpt (z1,1.8pt); drawpt (z2,1.8pt); drawpt (z3,1.8pt);

$z 5=(0,11.1 u) ; z 6=(8.9 u, 20 u) ; z 7=(31.1 u, 20 u) ;$

$z 8=(40 \mathrm{u}, 11.1 \mathrm{u}) ; \operatorname{drawpt}(\mathrm{z} 5,1.5 \mathrm{pt}) ;$

drawpt (z6,1.5pt); drawpt (z7,1.5pt);

drawpt (z8,1.5pt);

draw $z 1\{\operatorname{dir} 90\} \ldots z 2\{\operatorname{dir} 0\}$

..z3\{dir-90\}; \% ligne A

draw $z 1$.controls $z 5$ and $z 6 \ldots z 2$.

controls $z 7$ and $z 8 . . z 3 ; \%$ ligne $B$

draw $z 1\{$ dir90\}. . $\{$ dir-90\}z3;\% ligne $C$

draw z1\{dir20\}..z2\{dir45\}..\{dir-135\}z3;

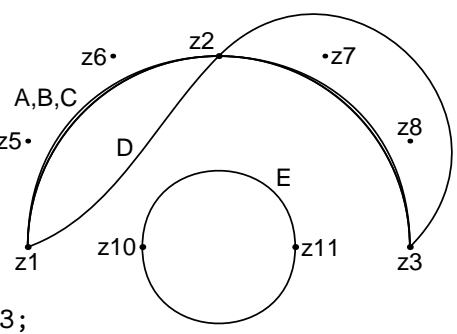

$\%$ ligne $\mathrm{D}$

$z 10=(12 u, 0) ; z 11=(28 u, 0) ; \operatorname{drawpt}(z 10,1.8 p t) ;$

drawpt (z11,1.8pt); draw z10..z11..cycle;\% ligne E

endfig;

\subsection{Modification du tRaCÉ : TENSION ET COURBURE}

On expose maintenant les options disponibles pour imposer des contraintes aux courbes telles qu'elles sont tracées ci-dessus, soit à partir des points guides seulement, soit à partir des points guides et des directions des tangentes en ces points. Ces possibilités sont au nombre de trois. Sur l'exemple de la page suivante où l'on trouvera le tracé normal et les tracés modifiés en trait plus fin (pour les deux premières possibilités : 
un tracé pour une valeur du paramètre plus faible que la valeur par défaut et un tracé pour la situation contraire; pour la troisième possibilité : un seul tracé avec modification).

- Dans le cas où on impose les directions des tangentes aux extrémités d'un segment, on peut, en modifiant le paramètre tension (défaut : 1 , valeur dans $[0.75, \infty]$ ), modifier le «gonflement» de la courbe (partie A). La syntaxe à utiliser [26], [M 15] : draw z0\{dir35\}.. tension $1.5 \ldots z 1\{$ dir -45$\}$;

- Dans le cas d'une extrémité d'une courbe ouverte où la direction de la tangente orientée n'est pas imposée, on peut modifier le paramètre curl (défaut : 1 , valeur dans $[0, \infty]$ ) pour modifier la courbe (partie B). Dans ce cas-ci, la dénomination du paramètre n'est pas très explicite; il caractérise la courbure au voisinage de l'extrémité concernée. Pour la valeur 1 , le tronçon de courbe au voisinage de $z 1$ est presque un arc de cercle d'un certain rayon, pour les valeurs plus grandes que 1, ce tronçon est voisin d'un arc de cercle d'un rayon plus petit et c'est le contraire pour les valeurs plus petites que 1 . La syntaxe correspondante est alors [27], [M 17] :

Figure 6 figc.mp

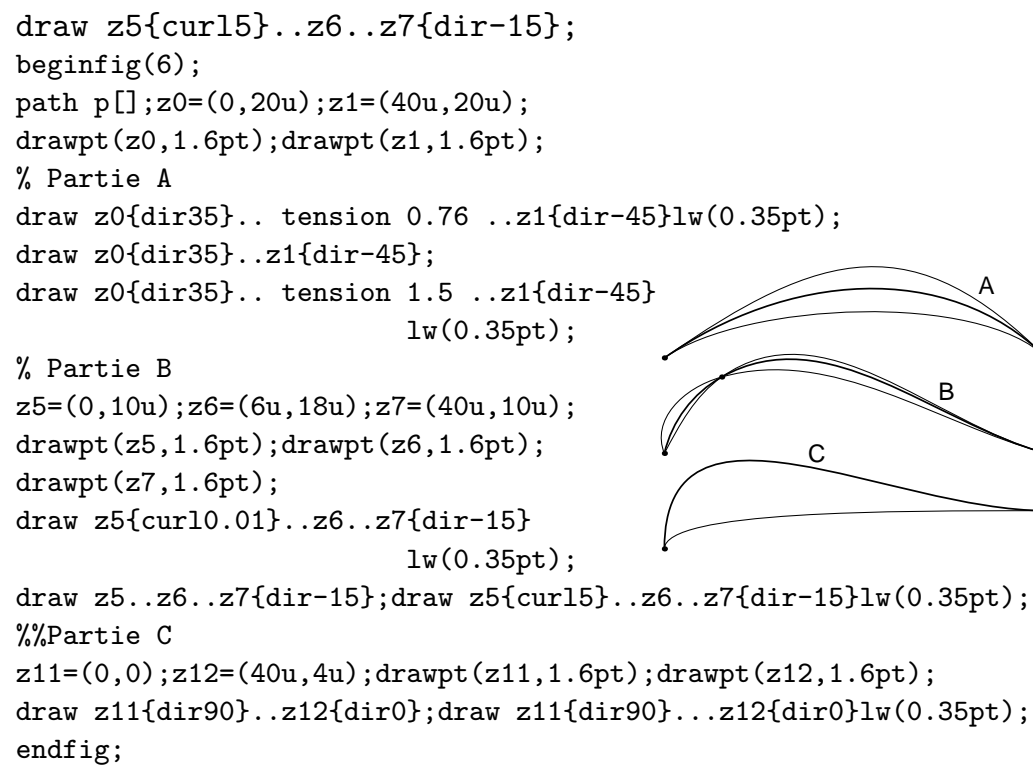

Cahiers GUTenberg no 52-53-Octobre 2009 
- De nouveau pour un tronçon de courbe avec les directions des tangentes aux extrémités imposées, on peut vouloir éviter un éventuel point d'inflexion; cela se fait en remplaçant les .. par ... dans la commande de tracé (partie $\mathrm{C}$ ) ; la syntaxe à utiliser est [26] :

draw z11\{dir90\}...z12\{dir0\};

\section{GESTION DES COURBES}

Cette section traite du découpage des courbes en élements, des intersections de courbes, de la construction de chemins fermés en vue d'un remplissage et des tangentes et des normales aux courbes. Avant d'entrer dans le vif du sujet il faut préciser la représentation paramétrique des courbes telle que l'utilise METAPOST. Une courbe de Bézier est représentée par un polynôme de Bernstein $\left(0 \leqslant t \leqslant 1, z_{1}, z_{2}, z_{3}, z_{4} \in \mathbb{C}\right)$ : $\mathrm{Z}(t)=(1-t)^{3} z_{1}+3(1-t)^{2} t z_{2}+3(1-t) t^{2} z_{3}+t^{3} z_{4}$

Une courbe ouverte ou fermée formée de $n$ éléments a encore une représentation paramétrique semblable avec $t \in[0, n+1]$. Le paramètre $t$ est parfois appelé temps car il est identifié au temps auquel un voyageur imaginaire passerait au point défini par $\mathrm{X}(t)$ et $\mathrm{Y}(t)$; cette identification explique les dénominations utilisées pour les commandes relatives à la gestion des courbes.

\subsection{Découpage des Courbes}

A partir d'une valeur de $t$, on peut obtenir les coordonnées $\mathrm{X}(t)$ et $\mathrm{Y}(t)$ du point correspondant de la courbe par la commande [57];

$z=$ point $t$ of $p$;

où $\mathrm{p}$ est le nom de la courbe. L'écriture $(\mathrm{a}, \mathrm{b})=$ point $\mathrm{t}$ of $\mathrm{p}$; donne directement les éléments de la paire représentant ce point. La partie de la courbe nommée pa et décrite par les valeurs de $t \in\left[t_{1}, t_{2}\right]$ est donnée par la commande [57] :

$\mathrm{pa}=$ subpath $(\mathrm{t} 1, \mathrm{t} 2)$ of $\mathrm{p}$;

Ces propriétes sont montrées sur l'exemple suivant. beginfig (7);

numeric $t[] ;$ path $p, p a, p a a ; z 1=(0,0) ; z 2=(15 u, 10 u) ; z 3=(30 u, 14 u)$;

drawpt (z1,1.8pt); drawpt (z2,1.8pt); drawpt (z3,1.8pt);

$\mathrm{p}=\mathrm{z} 1 . \mathrm{z} 2 \ldots \mathrm{z} 3$; draw $\mathrm{p} ; \mathrm{z} 4=$ point 1.3 of $\mathrm{p}$;

drawpt (z4,1.3pt);pa=subpath $(0.2,0.9)$ of $p$;

paa=pa shifted $(20 u, 0)$; draw paa $1 w(1.5 p t)$;

drawarrow $(10 u, 6 u)--(25.8 u, 6 u)$;

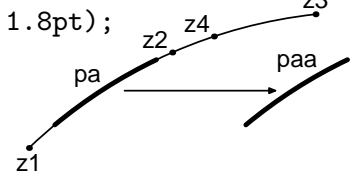

Figure 7

figc.mp

endfig; 


\subsection{INTERSECTIONS DE COURBES}

Le point d'intersection $\mathrm{z}$ des courbes pa et pb est donné par [55], [M 137] :

$\mathrm{z}=\mathrm{pa}$ intersectionpoint $\mathrm{pb}$;

s'il n'y a qu'un seul point d'intersection. On peut aussi utiliser [56] :

$(\mathrm{ta}, \mathrm{tb})=\mathrm{pa}$ intersectiontimes $\mathrm{pb}$;

qui donne les temps ta et tb correspondants au point d'intersection sur chacun des deux chemins. Ensuite, le point d'intersection z est obtenu avec:

$\mathrm{z}=$ point ta of pa; outb of $\mathrm{pb}$;

C'est cette deuxième méthode qui va être utilisée lorsqu'il y a plusieurs points d'intersection; l'exemple suivant montre les deux possibilités.

S'il n'y a aucun point d'intersection, la paire $(\mathrm{ta}, \mathrm{tb})$ vaut $(-1,-1)$; on peut voir que c'est la cas des chemins paa $=\operatorname{subpath}(0,1)$ of pa et $\mathrm{pb}$ en affichant à la console ces valeurs avec la commande :

Figure 8 figc.mp show ta, tb;

beginfig ( 8 );

$z 0=(0,0) ; z 1=(8 u, 10 u) ; z 2=(35 u, 19 u) ; z 3=(25 u, 0)$;

$z 4=(7 u, 20 u) ; z 5=(13 u, 0) ; z 6=(33 u, 24 u) ;$

drawpt (z0,1.8pt); drawpt (z1,1.8pt); drawpt (z2,1.8pt);

drawpt (z3,1.8pt); drawpt (z4,1.8pt);

drawpt (z5,1.8pt); drawpt (z6,1.8pt);

path pa,paa,pb,pc;pa=z0..z1..z2; draw pa;

$\mathrm{pb}=\mathrm{z} 3\{\operatorname{dir} 90\} \ldots \mathrm{z} 4\{\operatorname{dir} 155\}$;

draw pb dashed evenly;

z7=pa intersectionpoint pb; drawpt $(z 7,3 p t)$;

$\mathrm{pc}=\mathrm{z} 5\{$ dir90\} . z $6\{$ dir60\}; draw pc

dashed evenly scaled 2;

$(\mathrm{ta}, \mathrm{tb})=\mathrm{pa}$ intersectiontimes $\mathrm{pc}$;

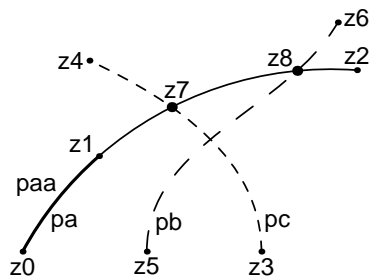

$z 8=$ point ta of pa;drawpt $(z 8,3 p t)$;

paa $=\operatorname{subpath}(0,1)$ of $\mathrm{pa} ;(\mathrm{tc}, \mathrm{td})=$ paa intersectiontimes $\mathrm{pb}$;

show tc,td;\% test de non intersection

endfig;

Il y a enfin le cas où l'on ne sait rien sur les positions des points d'intersection. Le programme METAPOST est conçu pour donner, de préférence, la paire $(\mathrm{ta}, \mathrm{tb})$ qui correspond au point d'intersection de $\mathrm{pa}$ et $\mathrm{pb}$ ayant la plus petite valeur de ta [56]. Cela permet de procéder comme suit :

— on commence par écrire l'équation :

Cahiers GUTenberg $n^{\circ}$ 52-53-Octobre 2009 
(ta1, tb1) $=$ pa intersectiontimes pb;

ce qui détermine le premier point d'intersection avec :

zint1=point ta1 of pa; ouzint1=point tb1 of pb;

- on recommence l'opération avec :

pa1=subpath (ta1+0.01, length of pa);

(ua2, tb2)=pa1 intersectiontimes $\mathrm{pb}$;

(sur la figure, on a pris 0.05 au lieu de 0.01 pour que l'effet soit visible : pa1 est plus épais que pa). Mais cette fois-ci, ua2 est une valeur du paramètre du sous-chemin pa1 et non une valeur du paramètre du chemin pa, donc le deuxième point d'intersection sera donné par :

zint2=point ua2 of pa1; (et non zint2=point ta2 of pa; que l'on aurait souhaité) ou zint2=point tb2 of $\mathrm{pb}$;

beginfig( 9$) ; \%$ !! IST = intersectiontimes

$z 0=(0,0) ; z 1=(40 u, 30 u) ; z 2=(12 u, 0) ; z 3=(6 u, 16 u) ; z 4=(28 u, 14 u)$;

Figure 9

$\mathrm{z} 5=(28 \mathrm{u}, 32 \mathrm{u}) ; \mathrm{path} \mathrm{pa}, \mathrm{pa}[], \mathrm{pb}, \mathrm{pb}[] ; \mathrm{pa}=\mathrm{z} 0\{\operatorname{dir} 50\} . \mathrm{z} 1\{\operatorname{dir} 25\}$;

figc.mp

$\mathrm{pb}=\mathrm{z2}$.z3..z4.z5; draw pa;draw pb;numeric ta[], ua [], tb [], ub [];

$\%$ Partie 1 : calcul de ta1, tb1, tb2 et tb3

$(\mathrm{ta} 1, \mathrm{tb} 1)=\mathrm{pa}$ IST $\mathrm{pb}$; show ta1,tb1;pa1=subpath $(\mathrm{ta1}+0.01,1)$ of pa;

(ua2,tb2)=pa1 IST pb; show ua2,tb2;pa2=subpath (ua2+0.01,1) of pa1;

(ua3,tb3)=pa2 IST pb; show ua3,tb3;pa3=subpath $(u a 3+0.01,1)$ of pa2;

(ua4,tb4)=pa3 intersectiontimes pb; show ua4,tb4;

drawpt (point tb1 of pb,2.5pt);

drawpt (point tb2 of pb,2.5pt);

drawpt (point tb3 of pb,2.5pt);

$\%$ Partie 2 : calcul de ta2 et ta3

pb1=subpath $(t b 1+0.01,3)$ of $\mathrm{pb}$;

draw pb1 lwcl;

(ub2, ta2) $=$ pb1 IST pa; show ta2, ub2;

pb2=subpath $(u b 2+0.01,3)$ of $\mathrm{pb} 1$;

(ub3,ta3) $=$ pb2 ITC pa; show ta3, ub3;

$\%$ Partie 3 : creation zones grisees

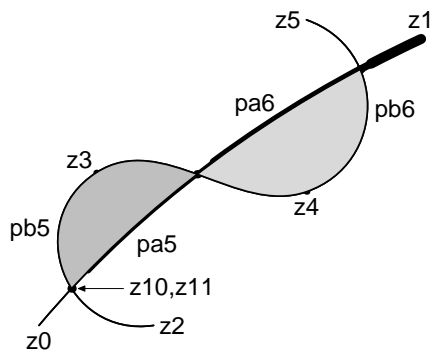

pa5=subpath (ta1, ta2) of pa;

$\mathrm{pb} 5=\operatorname{subpath}(\mathrm{tb} 1, \mathrm{tb} 2)$ of $\mathrm{pb}$;

pa6=subpath $(\mathrm{ta} 2, \mathrm{ta} 3)$ of $\mathrm{pa} ; \mathrm{pb} 6=\operatorname{subpath}(\mathrm{tb} 2, \mathrm{tb} 3)$ of $\mathrm{pb}$;

$\%<\operatorname{def} c l(\operatorname{expr} \mathrm{s})=$ withcolor $(\mathrm{s}, \mathrm{s}, \mathrm{s})$ enddef;

pb7=pa5. .reverse pb5. . cycle;fill pb7 cl(0.75);

pb8=pa6..reverse pb6. . cycle;fill pb8 cl(0.85); draw pa; draw pb;

$\%$ Partie 4 : verification de z10 different de z11

$z 10=$ point ta1 of pa; show $z 10 ; z 11=$ point tb1 of pb; show $z 11$;

endfig; 
- on arrête lorsque (uaj, tbj) $=(-1,-1$ ) (d'où la nécessité des commandes $\operatorname{show}(t a, t b)$ à chaque pas); on a alors déterminé les $j-1$ points d'intersection des chemins pa et pb (partie 1); on peut suivre sur la figure car les sous-chemins pa1, pa2 et pa3 sont de plus en plus épais.

- ainsi, on a bien les $j-1$ valeurs de tb avec lesquelles on peut découper des morceaux du chemin pb limités par des points d'intersection avec le chemin pa, mais on ne peut pas découper le chemin pa car on n'a que la première valeur ta1 de ta; les autres valeurs ta2 et ta3 sont nécessaires si l'on veut découper des morceaux de pa limités par des points d'intersection avec $\mathrm{pb}$; il suffit pour cela de recommencer le processus (partie 2) en interchangeant le rôle des chemins pa et pb.

On peut enfin nommer les morceaux des chemins pa et pb (pa5, pb5, etc.), puis former avec ces morceaux des chemins fermés (partie 3 ) avec, par exemple :

pb7=pa5 . reverse pb5 . cycle; fill pb7;

La nécessité de . . cycle (au lieu de l'opérateur de concaténation \&, voir sect. 3.5.3 et 4.2.6) est la conséquence des calculs approchés : le début z10 du chemin pa5 et la fin z11 du chemin reverse pb5 ne coïncident pas exactement.

On vérifie cette non coïncidence (partie 4) en calculant le même point d'intersection par $z 10$ point ta1 of pa; et $z 11$ point tb1 of $\mathrm{pb}$; qui donneraient le même résultat si les calculs étaient infiniment précis (la différence est de l'ordre de un millième de pt).

Pour s'éloigner au-delà du point d'intersection, on a arbitrairement choisi le temps ta1+0.01; METAPOST dispose pour cela d'un nombre très petit nommé epsilon [110], [M 62] valant 1/65536.

\subsection{CONSTRUCTION DIRECTE DES ZONES D'INTERSECTION}

Dans la sous-section précédente, on a construit puis coloré des zones que l'on peut appeller zones d'intersection; on a calculé les points d'intersection, puis formé des chemins partiels avec ces points et enfin construit les chemins fermés limitant ces zones. METAPOST dispose d'une puissante macro (qui n'existe pas dans METAFONT) permettant de définir ces zones d'intersection directement dans des cas particuliers auxquels on peut très souvent se ramener.

Le premier exemple de la page suivante correspond à la situation où deux chemins se coupent en deux points seulement. On va reprendre

Cahiers GUTenberg no 52-53-Octobre 2009 
l'exemple précédent où il y a trois points d'intersection mais où on peut limiter le chemin pb pour retomber dans la situation de validité de la macro; on écrit simplement [54] :

zoneint=buildcycle (chemin1, chemin2);

Il faut s'assurer de l'orientation compatible des chemins concernés. On rappelle qu'il faut remplir puis tracer les zones concernées (cf. section 2.1) ; si l'on est amené, lors de la construction d'une figure, à tracer des chemins pour suivre les étapes de la construction, il faudra peut-être en effacer une partie à la fin.

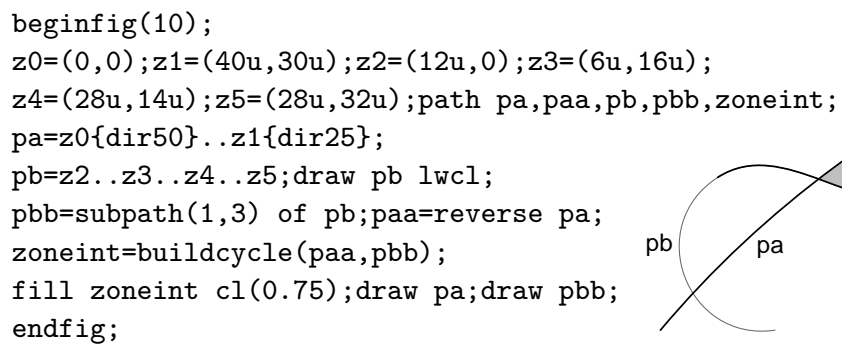

Le deuxième cas correspond à plus de deux chemins. Si l'on écrit par exemple la commande [54] : zoneint=buildcycle (p1,p2,p3);

METAPOST choisit le point d'intersection du chemin $p_{i}$ avec le chemin $p_{i+1}$ qui a la plus petite valeur du paramètre $t$ sur le chemin $p_{i}$ et la plus grande valeur sur le chemin $p_{i+1}$. On peut bien entendu obtenir des résultats surprenants sauf si les chemins $p_{i}$ et $p_{i+1}(\forall i, i=1, \ldots, n$, avec $p_{n+1}=p_{1}$ ) n'ont qu'un seul point d'intersection ou si l'on peut définir des sous-chemins ayant cette propriété. On reprend l'exemple de la figure 8 qui satisfait à cette condition.

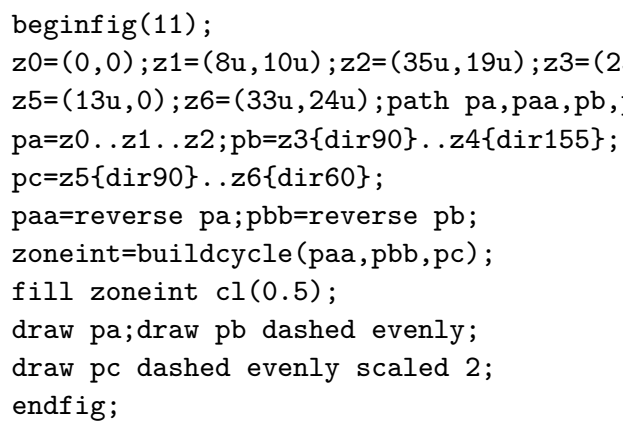

Figure 10

figc.mp

Figure 11

figc.mp 


\subsection{TANGENTES ET NORMALES AUX COURBES}

On peut tracer la tangente en un point de paramètre $t$ d'une courbe $p$ de la manière suivante, partie A de l'exemple [58] :

$(a, b)=$ direction $t$ of $p$;

donne les composantes a et $\mathrm{b}$ du vecteur direction de la tangente orientée à la courbe en ce point. Le point de contact est alors donné par :

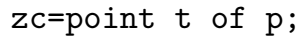

la tangente est alors définie avec $z c$ et le point $z c t=z c+(a, b)$ et la normale avec les points $z c$ et $z c n=z c+(-b, a)$.

On peut aussi tracer la tangeante à la courbe $\mathrm{p}$ au point d'abscisse $\mathrm{xc}$, partie B ; on définit alors le chemin rectiligne $\mathrm{pc}=(\mathrm{xc}, 0)--(\mathrm{xc}, 30 \mathrm{u})$ et alors la valeur du paramètre tc correspondant au point de contact $z c$ est donnée par [56] :

$(t c$, whatever $)=p$ intersectiontimes $q c$;

Figure 12 ce qui permet de tracer la tangente comme on l'a fait plus haut.

figc.mp beginfig(12); $z 0=(0,2 u) ; z 1=(20 u, 19 u) ; z 2=(32 u, 6 u) ; z 3=(40 u, 28 u) ;$ numeric tc;

path p,pp,droite, tanga, tangb, tangc;

$\mathrm{p}=\mathrm{z0}\{\operatorname{dir} 90\} \ldots z 1 \ldots z 2 \ldots z 3\{\operatorname{dir} 90\}$; draw $\mathrm{p}$;

$\%$ Donnée $t=0.8$; partie $A$ à gauche $(a, b)=$ direction 0.7 of $p ; z 4=$ point 0.7 of $p$; tanga $=1.2[z 4, z 4-(a, b)]--1.5[z 4, z 4+(a, b)]$; drawpt (z4,1.8pt); draw tanga;

$\%$ Donnée $\mathrm{xc}=27 \mathrm{u}$; partie $\mathrm{B}$ au milieu droite $=(27 u, 0)--(27 u, 30 u)$; draw droite $1 w(0.3 p t)$;

( $t c$, whatever $)=p$ intersectiontimes droite; $(c, d)=$ direction $t c$ of $p$;

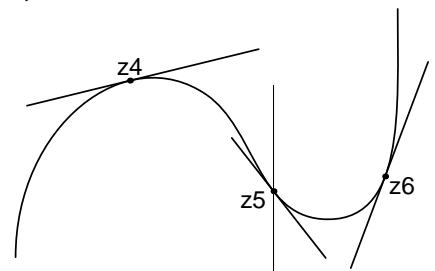
$z 5=$ point tc of p; tangb=1.2[z5, z5-(c,d)]--1.5[z5, z5+(c,d)]; drawpt (z5,1.8pt); draw tangb; $\%$ Donnée $(3 \mathrm{u}, 8 \mathrm{u})$; partie $\mathrm{C}$ à droite $\mathrm{pp}=\operatorname{subpath}(1,4)$ of $\mathrm{p}$; tcc $=$ directiontime $(3 \mathrm{u}, 8 \mathrm{u})$ of $\mathrm{pp}$; $\mathrm{z} 6=$ point tcc of pp; tangc=1.2[z6,z6-(3u,8u)]--1.5[z6,z6+(3u,8u)]; drawpt (z6,1.8pt); draw tangc; endfig;

La dernière possibilité consiste à tracer la tangente de direction donnée par un vecteur $(\mathrm{a}, \mathrm{b})$ à un chemin $\mathrm{p}$, partie $\mathrm{C}$ de l'exemple. La commande [58] :

Cahiers GUTenberg no 52-53 - Octobre 2009 
$\mathrm{tc}=\operatorname{directiontime}(\mathrm{a}, \mathrm{b})$ of $\mathrm{p}$;

donne la valeur tc (la plus petite valeur s'il y en a plusieurs possibles) du paramètre déterminant le point de tangence; le tracé de la tangente se fait alors comme ci-dessus. Il faut noter qu'il y a dans l'exemple cidessus deux possibilités pour tracer cette tangente de direction donnée : c'est pour cela que l'on a considéré le sous-chemin pp de p; ce type de méthode est conseillé car, très souvent, un simple examen visuel des chemins permet de choisir des sous chemins conduisant à une unique solution pour la recherche des points d'intersection ou, comme ici, pour un tracé de tangente.

Il reste un tracé non prévu par METAPOST : mener, à partir d'un point $z 3$, une tangente à un chemin p. On propose dans l'exemple suivant une méthode par approximations successives qui nous donne l'occasion de faire une boucle avec sortie conditionnelle. Pour ce petit exercice, on choisi d'abord un intervalle de variation de la valeur du paramètre $\mathrm{w}$ correspondant au point de contact; avec la valeur de $\mathrm{w}$, on construit la tangente dont on calcule le point d'intersection zinter avec l'axe des $x$. On balaie l'intervalle de variation dans le sens croissant : la valeur de w pour laquelle $\mathrm{xint}-\mathrm{x} 3$ change de signe donne le point de contact $\mathrm{zc}$ avec un précision que l'on peut apprécier en affichant, à chaque pas, ses coordonnées (de l'ordre de deux millièmes de pt).

beginfig(13);

$z 0=(0,0) ; z 1=(15 u, 0) ; z 3=(40 u, 0) ; p a t h p ; p=z 0\{\operatorname{dir} 90\} \ldots z 1\{\operatorname{dir}-90\}$;

Figure 13

figc.mp

draw p; draw $(-1 \mathrm{u}, 0)--(43 \mathrm{u}, 0) 1 \mathrm{w}(0.3 \mathrm{pt})$; drawpt $(z 3,2 \mathrm{pt})$;

for $w=0.53$ step 0.0001 until 0.59 :

numeric a,b,xinter;pair zc, zcc, zinter;

$(\mathrm{a}, \mathrm{b})=$ direction $\mathrm{w}$ of $\mathrm{p}$;

$z c=p o i n t w$ of $p ; z c c=z c+(a, b)$;

$\%$ show zc $\% \%$ Pour tester la precision

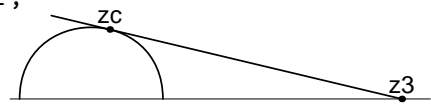

zinter $=$ whatever $[z c c, z c]=$ whatever $[z 0, z 1]$;

xinter=xpart zinter; exitif xinter<x3; endfor

draw 1.3[z3, zc]--z3; drawpt (zc,2pt);

endfig;

Sur cet exemple, il faut remarquer la présence à l'intérieur de la boucle des déclarations des variables $a, b$, xinter, zinter etc.; cette présence est nécessaire pour annuller les affectations faites au pas précédent de la boucle, sans quoi on aurait des messages d'erreur du type « inconsistent equations». Une autre solution serait de déclarer au début a [], b [], etc. 
et de travailler avec ces variables tableaux dans la boucle; mais toutes ces valeurs (des milliers si le pas est petit) sont inutiles, seule la dernière sert à construire la tangente. C'est une méthode à ne pas oublier.

\section{Compléments}

\subsection{Cercles et arcs de cercles}

On dispose des commandes [110-111] :

fullcircle, halfcircle et quatercircle

qui définissent respectivement (de diamètre $1 \mathrm{bp}$ et de centre $(0,0)$ ) un cercle complet, un demi-cercle et un quart de cercle. On écrit par exemple :

$p=$ fullcircle scaled $r$ shifted $z$;

pour avoir un cercle centré en $z$ et de rayon $r$. On construit une macro : $\operatorname{arcd}(z, r, a, b)(q)$

qui définit (mais ne trace pas) un arc de cercle nommé q centré en $z$, de

Figure 14

figc.mp rayon $r$, d'angle origine a et d'angle extrémité $b$.

beginfig (14);\%\% ROTAR=rotatedaroud

axespapiermilli $(-2.5,-2.5,40.5,21.5)$;

pair za, zb; path p,pcc, canne, tournant, triangle;

$\%<$ def $\operatorname{arcd}(\operatorname{expr} z, r, a, b)$ (text parg)=begingroup

$\%<$ save $c, p, p c ;$ path $p, p c ; c=b-a ; p=(0,0)--(1.2,0)$ rotated $c$;

$\%<\mathrm{pc}=$ fullcircle cutafter $\mathrm{p}$;

$\%<$ parg=pc scaled $\mathrm{r}$ rotated a shifted $\mathrm{z}$; endgroup enddef;

$\operatorname{arcd}((4 \mathrm{u}, 3 \mathrm{u}), 30 \mathrm{u}, 45,105)(\mathrm{pcc})$;

draw $\mathrm{pcc} ; \mathrm{p}=(0,0)--(17 \mathrm{u}, 0)$;

draw $p$ rotated 40 shifted $(4 u, 3 u) 1 w c l$;

draw $p$ rotated 100 shifted $(4 u, 3 u) 1 w c l$;

$\operatorname{drawpt}((4 \mathrm{u}, 3 \mathrm{u}), 2 \mathrm{pt})$;

$\operatorname{arcd}((15 u, 3 u), 6 u,-90,30)$ (tournant);

canne $=(0,0)--(15 u, 0)$ \&tournant;

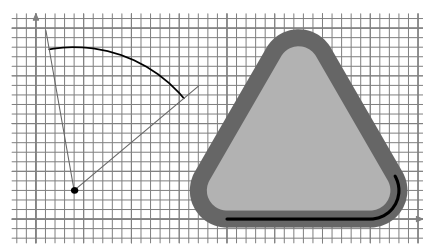

$\%$ draw canne; $\%$ test

$\mathrm{za}=(35 \mathrm{u}, 0)+(0,3 \mathrm{u})+\mathrm{polar}(3 \mathrm{u}, 30) ; \mathrm{zb}=(5 \mathrm{u}, 0)-(0,3 \mathrm{u})-\operatorname{polar}(3 \mathrm{u}, 30) ;$

triangle=canne shifted $(20 \mathrm{u}, 0)$. . canne shifted za ROTAR $(z a, 120)$. canne shifted zb $\operatorname{ROTAR}((20 u, 0),-120)$. .cycle;

fill triangle $\mathrm{cl}(0.7)$; draw triangle $l_{\mathrm{w}}(5 \mathrm{pt}) \mathrm{cl}(0.4)$;

draw canne shifted $(20 \mathrm{u}, 0) I_{\mathrm{w}}(1 \mathrm{pt})$;

endfig;

L'exemple ci-dessus utilise cette macro pour tracer un arc de cercle centré en $(4 u, 3 u)$, de rayon $6 u$, d'angle origine 40 degrés et d'angle

Cahiers GUTenberg no 52-53-Octobre 2009 
extrémité 100 degrés. Ensuite on dessine le «tableau » d'un panneau de signalisation routière (l'élément de base nommé canne apparaît en trait noir en bas du panneau). Ce code sera expliqué à la section 3.5.3.

\subsection{Autres COMmandes CONCERNANT LeS COURBES}

On signale d'abord que l'on a utilisé sans le dire le fait que tout ce qui concerne les intersections de chemins est encore valable si les chemins sont des droites (le polynôme $\mathrm{Z}(t)$ est alors du premier degré).

On a vu que l'on peut imposer la direction de la tangente orientée en chaque point guide des courbes mais on peut aussi imposer les directions des deux tangentes orientées de part et d'autre des points guides; pour cela on donne les valeurs des angles de part et d'autre du point-guide [25] :

draw etc..\{dir170\}z4\{dir-140\}..etc;

ce qui produit un point anguleux en $z 4$ (angle de 50 degrés).

La longueur paramétrique $l p$ et la longueur d'arc $l$ d'un chemin $p$ sont respectivement données par [57], [60] :

$l p=$ length $p$; et $l=a r c l e n g t h ~ p ;$

Par exemple, la longueur paramétrique de $\operatorname{subpath}(t 1, t 2)$ est $t 2-t 1$, celle d'une courbe ouverte passant par $n$ points guides est $n-1$.

La valeur $t z$ du paramètre qui définit le point $z$ du chemin $p$ est donnée par l'opérateur [60] :

t $z=\operatorname{arctime} z$ of $p$;

On dispose également de deux commandes qui permettent d'effacer la partie du chemin $\mathrm{p}$ se trouvant après (resp. avant) le point d'intersection avec le chemin q; la partie restante ppart est [58] :

ppart $=$ p cutafter $q$; (resp. cutbefore q;

$S$ 'il y a plusieurs points d'intersection entre les chemins, la partie supprimée est celle située après le point d'intersection ayant la plus grande valeur du paramètre dans le cas de cutaf ter et inversement dans la cas de cutbefore. On utilisera cette possibilité dans l'exemple suivant.

\subsection{EXPLiCATIONS CONCERNANT DES POINTS DE SYNTAXE}

Le dernier exemple, figure 14, ainsi que la macro arcd qui y est introduite, appellent les remarques importantes qui suivent.

On a déjà vu dans ces deux premiers chapitres des macros qui, en plus des paramètres dits « expressions» représentant des variables des 9 types 
possibles (dont seulement 6 ont été rencontrés à ce stade), contiennent des paramètres texte; dans le cas de la macro axespapiermillii, ce paramètre texte consiste en deux macros avec paramètres fixés. Dans le dernier exemple, la macro arcd a un paramètre texte qui est une liste de caractères : en effet, au moment où l'on définit cette macro arcd, parg n'est pas encore le nom d'un chemin mais une simple liste de caractères : cette liste ne peut donc pas faire partie du groupe de paramètres expressions.

On a défini plusieurs macros qui tracent des chemins ou des points (drawpt, drawrule, axespapiermillii, etc.); la macro arcd définit, sans le tracer, un chemin (un arc de cercle très précisemment) dont le nom est la liste de caractères donnée pour l'argument du type texte : dès que l'on a écrit la macro avec tous les arguments nécessaires, l'argument du type texte donné est devenu le nom de l'arc de cercle construit (la simple liste est devenue une variable chemin). Bien entendu on peut tracer ce chemin mais on peut aussi l'utiliser pour construire d'autres chemins comme cela est le cas dans l'exemple.

Dans cette macro arcd, on remarque les nouvelles commandes begingroup et endgroup qui limitent un groupement et la commande save dont le rôle va être expliqué sur un exemple. Dans le texte dit de remplacement de la macro arcd, on a besoin de variables intermédiaires; on prend pour exemple la variable $c=a-b$; : elle ne doit jouer aucun rôle en dehors de l'intérieur de la macro ; pour qu'elle ne perturbent pas le code environnant de la macro, la commande save c; sauvegarde la valeur de la variable c à l'entrée du groupement et la restitue à la sortie du groupement; il est évident que cela n'a d'utilité que si on utilise une variable nommée $c$ dans le code environnant, par contre, cela permet d'utiliser la macro sans avoir à connaître les noms des variables utilisées en interne. Après la commande save on trouve les déclarations faites suivant les règles habituelles.

La dernière remarque porte sur la construction du chemin fermé avec trois exemplaires du chemin nommé canne adhéquatement déplacés et tournés avec la translation shifted et la rotation rotatedaround. Les éléments du chemin composé sont concaténés avec . . au lieu de \& et la fermeture du chemin est assurée par l'option . .cycle; en effet, lors de la construction d'un chemin composé avec d'autres chemins, il se peut que l'extrémité d'un élément ne coïncide pas exactement avec l'origine

Cahiers GUTenberg no 52-53-Octobre 2009 
de l'élément suivant et il faut alors remplacer \& par . . sinon on a le message « \& will be changed to .. » qui précise que METAFONT a fait luimême le remplacement. On a aussi un tel exemple sur la figure 9 où les deux dernières commandes, partie 4 , permettent de voir que les points z10 et z11 ne coïncident pas rigoureusement. Pour voir le message, il suffit de remplacer .. par \& dans la ligne pa7=pa5 . reverse etc; et de regarder le message à la console. 



\section{CHAPITRE 4}

\section{La machinerie METAPOST}

Dans les deux premiers chapitres, on a appris à faire des figures simples assez facilement; cependant, au chapitre 3, l'écriture des commandes est devenue de plus en plus complexe et même un peu mystérieuse par certains aspects. METAPOST est un vrai langage informatique et, après les débuts destinés à motiver le lecteur, on doit aborder de façon logique et rigoureuse les éléments et la structure de ce langage. Nous allons examiner successivement les différents éléments en proposant toujours des exercices :

- les opérateurs,

- les variables,

— les variables internes,

- les équations et les affectations,

- les commandes,

- les comparaisons et les boucles,

- les macros.

\section{Opérateurs}

Les opérateurs produisent, à partir d'un ou deux éléments, un troisième élément; ces éléments sont les variables qui sont l'objet de la section suivante. Voilà des exemples d'opérateurs rencontrés dans les deux premiers chapitres :

1) nombre + nombre $=$ nombre

2) nombre $*$ nombre $=$ nombre

3) nombre $*$ paire $=$ paire

4) round nombre $=$ nombre

5) xpart paire $=$ nombre

6) $\operatorname{cosd}$ nombre $=$ nombre

7) nombre $<$ nombre = booléenne 
8) chemin intersectionpoint chemin = paire

9) point nombre of chemin = paire

10) subpath paire of chemin = chemin

11) chemin shifted paire $=$ chemin

12) paire shifted paire $=$ paire

On remarque d'abord qu'il y a des opérateurs qui prennent une variable et d'autres qui en prennent deux; tous donnent une variable en résultat. On reconnaît l'addition et la multiplication habituelle des nombres réels aux lignes 1) et 2). Dans ces deux lignes et dans la dernière, les trois variables sont du même type; à l'opposé il y a des cas où les variables concernées (deux ou trois suivant les opérateurs) sont de type différents; c'est le cas des lignes 5) et 9); il y a enfin les cas intermédiaires : lignes 7), $8), 10$ ) et 11) où deux des trois variables sont d'un même type.

La liste des opérateurs avec les arguments qu'ils peuvent prendre, les résultats qu'ils peuvent donner et l'explication de leur action se trouve dans un long tableau du manuel cité [112-119]; le lecteur s'y reportera chaque fois qu'il voudra une précision concernant un opérateur.

\section{VARIABLES}

Il faut noter que, dans les exercices suivants (fichier de figures figd.mp), on doit parfois déclarer les variables nombre dont la déclaration est facultative (cas où ces variables sont utilisées plusieurs fois). Cette déclaration, à faire dès la deuxième utilisation, efface la valeur de la variable correspondante si elle a déjà été utilisée auparavant, en particulier dans une figure précédente. Cette remarque est sans objet pour les variables $\mathrm{z}[], \mathrm{x}[]$ et y [] dont les valeurs sont effacées par la commande beginfig. Pour les autres variables, leur redéclaration assure de la même manière l'effacement des valeurs prises antérieurement.

On va examiner les neuf types de variables en donnant leurs propriétés (si ce n'est déjà fait) et les principaux opérateurs qui peuvent leur être associés.

On commence par une commande et deux opérateurs qui concernent toutes les variables et qui sont utiles dans le débogage :

show a;

montre à la console la valeur de la variable a quel que soit son type [28] ; en particulier, si $a=" A B "$;, alors show $a$; et show "AB"; affichent $A B$ (début de l'exercice 1, page suivante);

Cahiers GUTenberg no 52-53-Octobre 2009 
known a;

vaut true si la variable a a une valeur particulière et false dans le cas contraire [39] : autrement dit show known a affiche respectivement true ou false (milieu de l'exercice);

numeric a;

vaut true si la variable a est du type nombre et false dans le cas contraire [39] ; on dispose des huit opérateurs similaires pour les huit autres types de variables : pair, color, etc. (fin de l'exercice).

Dans ce chapitre, on va trouver des exercices « à la console » dont le résultat va s'afficher à l'écran; s'il y a trop de lignes, il faut éditer le fichier figd. log pour voir l'ensemble des résultats. Voilà le premier.

Exercice 1

figd.mp

beginfig(1); show "Exercice 1";

$a=1.25$; show a; pair $z z ; z z=(5,2.5)$; show $z z$; show red;

path $\mathrm{p} ; \mathrm{p}=(0,0)--(1,0)$; show $\mathrm{p}$;

transform $\mathrm{T}$; $\mathrm{T}=$ identity $\operatorname{shifted}(10,0)$; show $\mathrm{T}$;

show known $b ; b=5$; show known $b$;

show known q; path q; show known q; $q=(0,0)--(0,1)$; show known $q$;

show pair a;show numeric a;show numeric zz;show pair zz;show zz;

endfig;

\subsection{TYPE NOMBRE}

Principaux opérateurs du type $a \mathrm{o}_{\mathrm{p}} b \rightarrow c$ et $\mathrm{o}_{\mathrm{p}} a \rightarrow c$ où $a, b$ et $c$ sont des variables du type nombre [33] (début de l'exercice 2) :

- addition, soustraction, mutiplication, division (sauf par 0), division entière, addition pythagoricienne, exponentiation, $(+,-, *, /, \operatorname{div},++$, $* *)$,

- racine carrée, valeur absolue, plus petit entier supérieur ou égal, plus grand entier inférieur ou égal, cosinus et sinus (angle en degrés), plus proche entier (sqrt, abs, ceiling, floor, cosd, sind, round).

Principaux opérateurs de comparaison du type $a \mathrm{o}_{\mathrm{p}} b \rightarrow \mathfrak{b}$ ou $\mathrm{o}_{\mathrm{p}} b \rightarrow \mathfrak{b}$ où $a$ et $b$ sont des variables nombre et $\mathfrak{b}$ est une variable du type booléen (fin de l'exercice) :

- égalité, plus petit, plus grand, différent, plus petit ou égal, plus grand ou égal, imparité $(=,\langle\rangle,,\langle>,\langle=\rangle=$,, odd).

D'autres opérateurs prenant ou donnant des variables du type nombre et aussi des variables d'autres types seront présentés avec ces autres 


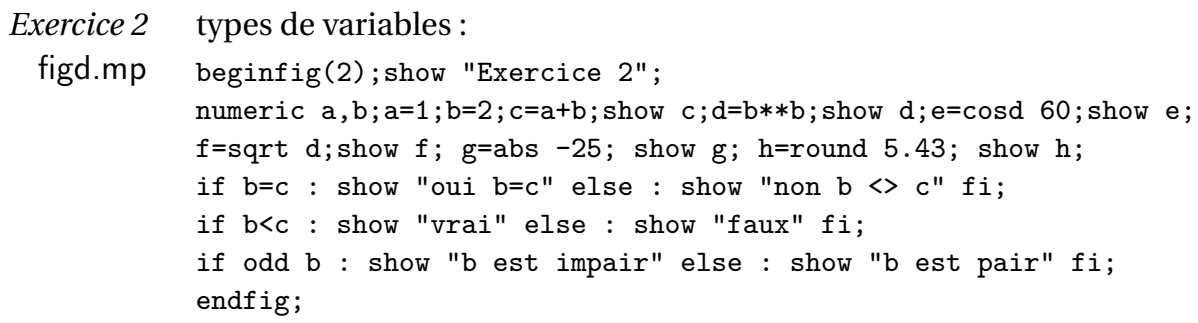

\subsection{TYPE PAIRE}

Opérateurs du type $z_{a} \mathrm{o}_{\mathrm{p}} z_{b} \rightarrow z_{c}$ et $a \mathrm{o}_{\mathrm{p}} z_{a} \rightarrow z_{c}$ où $z_{a}, z_{b}$ et $z_{c}$ sont des variables du type paire [34] et $a$ est une variable nombre (début de l'exercice 3) :

- addition, soustraction, multiplication par variable nombre et division par une variable nombre différente de $0(+,-, *, /)$.

Principaux opérateurs de comparaison du type $z_{a} \mathrm{o}_{\mathrm{p}} z_{b} \rightarrow \mathfrak{b}$ où $\mathfrak{b}$ est une variable booléenne (milieu de l'exercice) :

- égalité, différent $(=,<>)$.

Principaux autres opérateurs (fin de l'exercice) :

- xpart $z$, ypart $z$, abs $z$, round $z$ donnent respectivement le premier nombre de la paire $z$, le deuxième nombre de $z$, le module de $z$, la paire des deux plus proches entiers des deux nombres de $z$.

- angle $z$, unit vector $z$, dir u donnent respectivement l'angle que le vecteur de composantes xpart $\mathrm{z}$ et ypart $\mathrm{z}$ fait avec l'axe des $x$, la paire des composantes du vecteur unitaire suivant le vecteur de composantes xpart $z$ et ypart $z$, la paire des composantes du vecteur uni-

Exercice 3 taire faisant l'angle u avec l'axe des $x$.

figd.mp

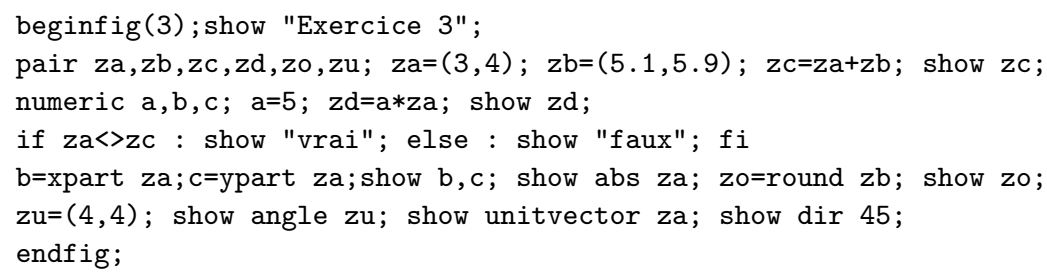

\subsection{TYPE COULEUR}

Les couleurs sont codées à l'aide du système RVB : une variable du type couleur [34] est un triplet ( $a, b, c)$ de trois nombres inférieurs ou égaux à 1 .

Cahiers GUTenberg no 52-53 - Octobre 2009 
Opérateurs du type du type $c_{a} \mathrm{o}_{\mathrm{p}} c_{b} \rightarrow c_{c}$ et $a \mathrm{o}_{\mathrm{p}} c_{a} \rightarrow c_{c}$ où $c_{a}, c_{b}$ et $c_{c}$ sont des variables du type couleur et $a$ est une variable nombre :

- addition, soustraction, multiplication par une variable nombre et division par une variable nombre différente de $0(+,-, *$ et /).

Principaux opérateurs de comparaison du type $c_{a} \mathrm{o}_{\mathrm{p}} c_{b} \rightarrow \mathfrak{b}$ où $\mathfrak{b}$ est une variable booléenne :

- égalité, différent $(=,<>)$.

Autres opérateurs éventuellement utiles :

- redpart c, greenpart c et bluepart c donnent respectivement les composantes rouge, verte et bleue de la couleur c.

Il est possible que les différentes opérations faites sur les couleurs conduisent à des triplets dont un ou plusieurs éléments dépassent 1; dans ce cas, METAPOST multiplie le triplet par le nombre qui ramène à 1 le plus grand élément du triplet; par exemple ${ }^{3}$ : $(0.8,0.6,0.2)+(0.7,0.6,0.4)=(1.5,1.2,0.6)$ sera multiplié par $1 / 1.5$ ce qui donne $(1,0.8,0.4)$.

\subsection{TYPE TRANSFORMATION}

D'une façon complètement générale, on peut représenter une transformation affine du plan par un sextuplet $\left(t_{x}, t_{y}, t_{x x}, t_{x y}, t_{y x}, t_{y y}\right)$ tel que :

$$
(x, y) \rightarrow\left(t_{x}+t_{x x} x+t_{x y} y, t_{y}+t_{y x} x+t_{y y} y\right)
$$

Heureusement, les transformations utiles peuvent s'obtenir très simplement à partir des transformations élémentaires suivantes [60-63], [M 141-145] (début de l'exercice 4, deuxième page suivante) :

- $\operatorname{shifted}(\mathrm{a}, \mathrm{b})$ : translation de $a$ suivant les $x$ et de $b$ suivant les $y$ (translation de vecteur $(a, b)$ souvent utilisée, exemple : fig. 1.4, sect. 1.3),

- rotated $\mathrm{u}$ : rotation autour de l'origine d'un angle $u$ (sens positif habituel),

- slanted a : $(x, y) \rightarrow(x+a y, y)$ (utilisée initialement pour pencher les caractères),

- scaled a : $(x, y) \rightarrow(a x, a y)$, agrandissement ou réduction suivant les valeurs du facteur d'échelle $a$,

3. Autre exemple : $(3,3,3)$ sera remplacé par $(1,1,1)$ car il ne peut y avoir de blanc plus blanc que blanc, excepté dans le monde des lessives comme l'avait, en son temps, remarqué Coluche! 
- xscaled a $:(x, y) \rightarrow(a x, y)$, changement d'échelle suivant les $x$ seulement,

- yscaled a : changement d'échelle suivant les $y$ seulement,

- zscaled (a, b) : rotation et homothétie qui transforme $(0,1)$ en $(a, b)$ (rotation d'un angle égal à l'angle que le vecteur $(a, b)$ fait avec l'axe des $x$ suivie d'une homothétie de facteur $\sqrt{a^{2}+b^{2}}$ ),

- rotatedaround $(\mathrm{z}, \mathrm{u})$ : rotation d'angle $u$ autour du point $z$,

- reflectedabout $(z 1, z 2)$ : réflection par rapport à la droite définie par les points $z_{1}$ et $z_{2}$.

On retrouve un résultat bien connu (milieu de l'exercice) : rotatedaround $(\mathrm{z}, \mathrm{u})$

donne le même résultat que shifted $-z$ suivie de rotated $u$ suivie de shifted $z$ Le chemin transformé pp s'écrit, si p est le chemin initial :

- $p$ shifted $-z$ rotated $u$ shifted $z$ L'application d'une succession de transformations prédéfinies ci-dessus, s'écrit donc sans notation spécifique.

Les transformations peuvent être appliquées à une paire représentant un point du plan, à un chemin ou à un dessin ; on obtient alors respectivement une autre paire représentant un autre point du plan, un autre chemin ou un autre dessin : on constate alors que les transformations jouent le rôle d'un opérateur du type qui intervient dans une équation telle que $p \mathrm{o}_{\mathrm{p}} \rightarrow p$ où $p$ est un point, un chemin ou un dessin.

Mais les transformations sont aussi des variables [34] que l'on déclare, définit et utilise comme dans les exemples suivants :

- transform Ta, Tb, Tc, T[];

- T=identity shifted za rotated u reflectedabout (zb,zc);

- $z b=z a$ transformed Ta; pb=pa transformed Ta; (idem avec un dessin: desb=desa transformed $\mathrm{Ta}$ ),

- Tc=Ta transformed $\mathrm{Tb}$;

- inverse Ta est la transformation inverse de Ta.

L'avant dernière ligne (fin de l'exercice) montre la loi de composition (non commutative ${ }^{4}$ ) des transformations avec l'opérateur

4. Le lecteur se souvient probablement de l'heureux temps où un professeur lui a fait découvrir qu'une loi de composition n'est pas nécessairement commutative avec l'exemple d'une rotation et d'une translation!

Cahiers GUTenberg no 52-53-Octobre 2009 
transformed. La dernière ligne définit la transformation inverse avec l'opérateur inverse (fin de l'exemple); dans l'exemple choisi, on ne retrouve pas exactement la transformation identité à cause des approximations de calcul (le lecteur doit commencer à être familier avec ces effets).

Exercice 4

beginfig(4); show "Exercice 4";

figd.mp

show "debut (exemples de transformations)";

show identity;

show identity shifted $(20 \mathrm{u}, 10 \mathrm{u})$; show identity rotated 45;

show identity rotatedaround $((10 \mathrm{u}, 30 \mathrm{u}), 60)$;

show "milieu (remarque)";

show identity rotatedaround $((20 \mathrm{u}, 10 \mathrm{u}), 45)$;

show identity shifted-(20u,10u) rotated 45 shifted(20u,10u);

show "fin (composition sur l'exemple de la remarque)";

pair zr;transform $\mathrm{Ta}, \mathrm{Tb}, \mathrm{Tc}, \mathrm{Td}, \mathrm{Te} ; \mathrm{zr}=(20 \mathrm{u}, 10 \mathrm{u})$;

$\mathrm{Ta}=$ identity shifted $-\mathrm{zr} ; \mathrm{Tb}=$ identity rotated 45 ;

Tc=identity shifted $\mathbf{z r}$;

$\mathrm{Td}=\mathrm{Ta}$ transformed $\mathrm{Tb}$ transformed $\mathrm{Tc}$; show $\mathrm{Td}$;

$\mathrm{Te}=\mathrm{Td}$ transformed inverse $\mathrm{Td}$; show $\mathrm{Te}$;

endfig;

Du point de vue mnémotechnique, il suffit de se souvenir que les transformations autres que les transformations prédéfinies (ci-dessus) sont utilisées précédées de transformed et que toute définition d'une transformation avec les transformations prédéfinies débute par la transformation identity.

\subsection{TyPe CHEMIN}

Les variables du type chemin [34] peuvent:

- être transformés par les transformations définies à la sous-section précédente, par exemple $: \mathrm{p}$ shifted $(\mathrm{a}, \mathrm{b})$ donne le chemin $\mathrm{p}$ déplacé de a suivant les $x$ et de b suivant les $y$,

- être concaténés par l'opérateur \& : si pa et pb sont des chemins, alors pa \& pb est un chemin si et seulement si l'extrémité de pa coïncide avec l'origine de $\mathrm{pb}$; il peut arriver que cette coïncidence ne soit pas exacte (arrondis de calcul); dans ce cas, si l'on veut former un chemin fermé (par exemple pour être rempli), alors METAPOST remplace \& par .., ce qui est invisible (le chemin rajouté entre les points qui ne coïncident pas n'est long que de quelques dix millièmes de pt, c'est le cas dans la figure 
3.9 du chapitre 3 , chemins pb7 et pb8),

- définir un chemin fermé grâce à la macro buildcycle (pa,pc, etc) (sect. 3.4.3) sans avoir à calculer les points d'intersection de ces chemins. On dispose aussi d'un opérateur cycle [39] pour tester si un chemin est ou n'est pas fermé; de toute manière, si l'on tente de remplir (avec $f i l l$ ) un chemin non fermé, METAPOST le signale.

Autres opérateurs déjà rencontrés :

- point $t$ of $\mathrm{p}$ (sect. 3.4.2) donne le point de $\mathrm{p}$ au temps $t$,

- $\operatorname{subpath}(t 1, t 2)$ of $p$ (sect. 3.4.1) donne la partie de $p$ comprise entre les points aux temps $t 1$ et $t 2$,

- pa intersectionpoint pbet pa intersectiontimes $\mathrm{pb}$ (sect. 3.4.2) permettent de trouver les points d'intersection des chemins pa et $\mathrm{pb}$,

- pa cutafter pb et pa cutbefore pb (sect. 3.5.2) permettent de gommer la partie de pa située après et avant le point d'intersection avec $\mathrm{pb}$,

- direction $t$ of $p$ et directiontime $t$ of $p$ (sect. 3.4.4) permettent les tracés de tangentes au chemin $p$;

- length $\mathrm{p}$, arclength $\mathrm{p}$ et arctime $\mathrm{p}$ (sect. 3.5.2) servent à gérer le chemin $\mathrm{p}$.

\subsection{TYPE CHAÎNE}

Les valeurs sont attribuées aux variables du type chaîne [35] en les limitant par des double-quote : après déclaration, on écrit par exemple : $\mathrm{ch}=$ "abcdef ";

Principaux opérateurs [112-119] :

- opérateur de concaténation \&,

- opérateurs de comparaison : égalité , différent, plus petit, plus grand, plus petit ou égal, plus grand ou égal $(=,\langle>,<,>,<=\mathrm{et}\rangle=)$; dans les quatre derniers cas, c'est le code ASCII du premier caractère qui est seul pris en compte.

Autres opérateurs utiles présentés dans l'exemple de la page suivante :

- ASCII ch; donne le code ASCII du premier caractère de ch,

- char $\mathrm{n}$; donne le caractère de code $n$,

- substring $(a, b)$ of $c h$; extrait la sous-chaîne dont le premier caractère est à la position $a$ et le dernier à la position $b-1$

Cahiers GUTenberg no 52-53-Octobre 2009 
(attention : dans les chaînes, la première position est la position 0 , substring $(2,4)$ "abcde" donne cd),

- label (ch , z) ; permet d'écrire la chaîne ch à la position z.

- str aaa; (où aaa est une simple liste de caractères) donne la chaîne "aaa".

Exercice 5

beginfig(5); show "Exercice 5"

figd.mp

string $\mathrm{s}[] ; \mathrm{s} 1=" \mathrm{ab} " ; \mathrm{s} 2=" \mathrm{cde} " ; \mathrm{s} 3=\mathrm{s} 1 \& \mathrm{~s} 2$; show $\mathrm{s} 3$;

s5="ab"; if s1=s5 : show "vrai" else : show "faux" fi;

s6="ac"; if s1=s6 : show "vrai" else : show "faux" fi;

s7="bb"; if s1<s7 : show "vrai" else : show "faux" fi;

show ASCII "ABC"; show char65;s8=substring $(2,4)$ of "abcde"; show s8;

s9=str uuu; show s9;

endfig;

\subsection{TYPE BOOLÉEN}

Les variables booléennes [35] apparaissent la plupart des fois comme le résultat d'un opérateur de comparaison (exemple : fig. 3.13) ou d'un opérateur de test (known, numeric, path, etc., sect. 4.2). On les compose avec les opérateurs [112-119] and et or ; la négation s'obtient avec l'opérateur not (début exercice suivant). On peut leur attribuer des valeurs en utilisant les parenthèses comme délimiteurs; par exemple, après déclaration, on écrit (fin exercice) :

- $b a=(a<b) ; b b=(b<c)$; puis if $b a$ and $b b$ : etc ;

beginfig(6); show "Exercice 6";

$\mathrm{k}=1 ; \mathrm{l}=3 ; \mathrm{m}=5 ; \mathrm{n}=2$;

if $(\mathrm{k}<\mathrm{l})$ and $(1<\mathrm{m})$ : show "ordre" else:show "desorde" $f i$;

if $(k<1)$ and $(1<n)$ :show "ordre" else:show "desorde" $f i$;

if $(k<1)$ and not $(l<m)$ : show "ordre" else:show "desorde" $f i$;

boolean ba, bb, bc; ba $=(\mathrm{k}<1) ; \mathrm{bb}=(1<\mathrm{m}) ; \mathrm{bc}=\mathrm{ba}$ and $\mathrm{bb}$; show $\mathrm{bc}$;

endfig;

\subsection{TyPE DESSIN}

Un ensemble de chemins tracés ou remplis constitue un dessin. Après déclaration, on peut attribuer à une variable de type dessin [35] une «valeur» constituée par un dessin donné.

On utilise la variable dessin prédéfinie nommée currentpicture pour cela [74], [M 114] ; elle contient tout ce qui a été tracé ou rempli lorsque la variable est invoquée (elle a été utilisée pour découper une

Exercice 6

figd.mp 
partie du dessin préalablement fait, figure 2.2 du chapitre 2). On dispose aussi de la variable dessin prédéfinie nullpicture qui contient toujours un dessin vide.

On donne la «valeur» constituée par les chemins qui ont été précédemment tracés ou remplis à la variable dessin préalablement déclarée en écrivant la suite de commandes suivante :

- picture $D ; D=$ currentpicture; currentpicture:=nullpicture; Après cette ligne, la variable $\mathrm{D}$ contient le dessin préalablement fait et la variable currentpicture ne contient plus de dessin : en d'autre termes si currentpicture représente le contenu du tableau, la première partie de la ligne fait une copie nommée $\mathrm{D}$ de ce qu'il y a sur le tableau; la seconde partie efface le tableau où l'on peut commencer un autre dessin!

Dans l'exemple suivant, on montre comment on peut préparer des éléments d'une figure très simplement, puis les assembler pour former la figure complète : le principe de base consiste à faire un élément, le sauvegarder dans une variable dessin, égaler currentpicture à nullpicture "pour effacer le tableau " et construire l'élément suivant, etc.; quand tous les éléments sont terminés, on les assemble par concaténation avec la syntaxe suivante (étant entendu que la variable currentpicture a été complètement «vidée») [73], [M 144] :

- addto currentpicture also $\mathrm{Pa}$; addto currentpicture also $\mathrm{Pb}$ shifted za; addto currentpicture also etc ;

Exercice 7

figd.mp et ainsi de suite jusqu'à la construction complète de la figure.

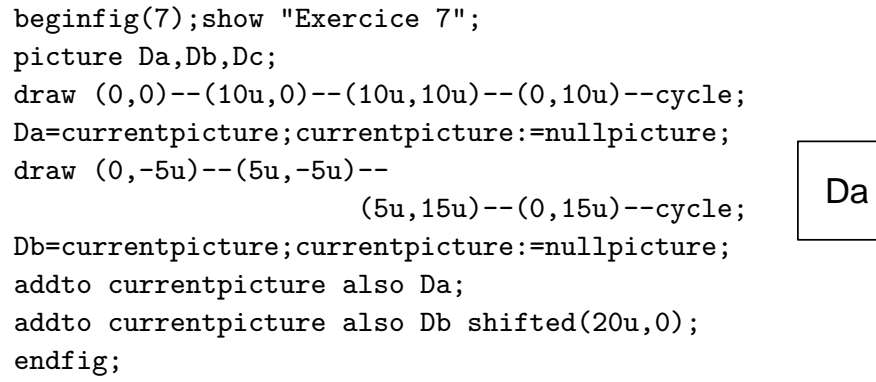




\subsection{TyPe Plume}

Ce type de variable n'est pas abordé dans ce manuel puisque l'on s'est fixé l'objectif de faire des figures avec simplement des traits d'épaisseur constante, ce qui s'obtient avec la plume par défaut (pencircle) dont on fait varier le diamètre avec une option du type (sect. 2.2.1) :

- withpen pencircle scaled 0.8pt

\section{VARIABLE ET ÉQUATIONS ; VARIABLES INTERNES ET AFFECTATIONS}

Il est grand temps de faire le point!

\subsection{VARIABLES (ORDINAIRES)}

Ce sont les variables déjà examinées dans la section 4.2 : elles sont d'abord déclarées (sauf dans quelques cas bien précisés, (sect. 2.3.3) puis déterminées par METAPOST avec les équations que l'on écrit (exercice suivant avec des paires) :

- $z 1=(3,5)$; METAPOST résout l'équation et attribue la valeur $(3,5)$ à la variable $z 1$ (semble tout à fait trivial!) ;

- $z 1+z 2=(5,9) ; z 1-z 2=(3,3) ; \operatorname{METAPOST}$ attribue la valeur $(4,6)$ à $z 1$ et la valeur $(1,3)$ à $z 2$ (c'est moins trivial!) ;

- $z 1+z 2=(5,9) ; z 1-z 2=(3,3) ; z 1=(4,3)$; va donner un message d'incompatibilité car les deux premières équations déterminent une valeur de z1 différente de celle donnée par la dernière équation; par contre, si la dernière équation est $z 1=(4,6)$, il n'y aura pas de message d'erreur ;

- pair za,zb;za+zb=(5,9);za-zb=(3,3);pair za;za= $(4,3)$ ne va pas donner de message d'erreur car la déclaration pair za; fait que METAPOST oublie la valeur qu'il a attribuée à za; cette propriété permet, dans une boucle où les valeurs intermédiaires des variables n'ont aucune utilité après la boucle, d'utiliser les mêmes variables à chaque pas de la boucle à condition de les redéclarer dans la boucle avant leur nouvelle utilisation (voir figure 3.13, sect. 3.4.4)

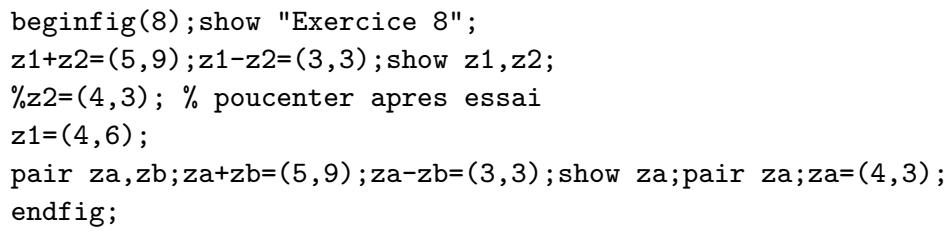

Exercice 8

figd.mp 


\subsection{VARIABLES INTERNES}

Certaines variables internes sont prédéfinies et d'autres sont introduites par l'utilisateur à l'aide de la déclaration newinternal; leur valeur, nulle à leur déclaration, est donnée, on dit affectée, par l'utilisateur ; voici quatre exemples :

- ahlength est une variable interne (valeur de la longueur de la pointe des flèches, sect. 2.2.4) dont la valeur par défaut est $4 \mathrm{bp}$; on l'a changée en écrivant ahlength $=2 p t$ dans le préambule du fichier des figures du chapitre 2 ; on peut revenir à la valeur par défaut en écrivant ahlength: $=4 \mathrm{bp}$; une autre possibilité consiste à écrire ahlength $:=$ ahlength $-2 \mathrm{bp}$ et ahlength $:=$ ahlength $+2 \mathrm{bp}$ pour retrouver la valeur initiale;

- linecap est une variable interne (forme de la terminaison des traits, sect. 2.2.2) dont la valeur par défaut est rounded mais on peut aussi choisir une autre valeur en écrivant linecap:=squared; ici butt, rounded et squared sont des constantes prédéfinies que peut prendre la variable interne linecap (l'intérêt de la définition de ces constantes, qui valent respectivement 0,1 , et 2 , est simplement d'ordre mnémotechnique) ;

- currentpicture est une variable interne prédéfinie qui contient l'ensemble des chemins tracés ou remplis précédemment (sect. 2.1 et 4.2.8);

- newinternal dist; dist:=3pt; définit une variable interne utilisateur représentant une distance pour une construction spécifique (par exemple, distance entre les deux filets d'un double cadre).

Les variables internes prédéfinies et les constantes prédéfinies sont respectivement données par les tableaux du manuel cité [107-109] et [110-111].

\subsection{REMARQUES CONCERNANT LES AFFECTATIONS}

Les variables internes, prédéfinies ou définies par l'utilisateur, sont toujours affectées au niveau METAPOST pour les premières et par l'utilisateur pour les secondes. On change à la demande leur valeur par une nouvelle affectation $(:=)$.

On peut aussi affecter une valeur à une variable (ordinaire), voici quelques cas possibles proposés surtout dans un but pédagogique (exercice page suivante) :

- si x1 est déterminée par des équations et vaut 4 et si on écrit x $1:=5$;

Cahiers GUTenberg no 52-53-Octobre 2009 
ou $\mathrm{x} 1:=\mathrm{x} 1+1$; après les équations, alors $\mathrm{x} 1$ prend la valeur 5 ;

- si on écrit $\times 11:=5$; avant les équations qui donnent pour solution $x 11=4$; , on a un message d'incompatibilité mais, si on écrit $x 21:=4$; avant les équations qui donnent pour solution $\times 21=4$; alors on a un message de redondance.

- si on écrit $x 31:=x 31+1$; avant les équations qui déterminent $x 31$ et x32, ces équations donnent encore la même solution; en effet, comme x31 n'a pas encore de valeur, l'équation $x 31:=x 31+1$; ne peut lui en donner une; d'où la réponse "false" à la commande show known x31;

beginfig(9); show "Exercice 9";

Exercice 9

$\mathrm{x} 1+\mathrm{x} 2=7 ; \mathrm{x} 1-\mathrm{x} 2=1$;

show $\mathrm{x} 1, \mathrm{x} 2 ; \mathrm{x} 1:=5$; show $\mathrm{x} 1$;

$\% \mathrm{x} 11:=5 ; \mathrm{x} 11+\mathrm{x} 12=7 ; \mathrm{x} 11-\mathrm{x} 12=1 ;$ show $\mathrm{x} 11, \mathrm{x} 12 \%$ pourcenter apres essai

$\mathrm{x} 21:=4 ; \mathrm{x} 21+\mathrm{x} 22=7 ; \mathrm{x} 21-\mathrm{x} 22=1 ;$ show $\mathrm{x} 21, \mathrm{x} 22$;

$\mathrm{x} 31$ : $=\mathrm{x} 31+1$; show known $\mathrm{x} 31 ; \mathrm{x} 31+\mathrm{x} 32=7$;

$\mathrm{x} 31-\mathrm{x} 32=1$; show $\mathrm{x} 31, \mathrm{x} 32$;

endfig;

\section{Commandes et macros}

\subsection{Commandes}

On va donner la liste des commandes déjà utilisées ou qui vont être utilisées dans ce document. Pour débuter en METAPOST, on peut se limiter a une partie de la liste complète de ces commandes [120]. En effet, cette liste contient des primitives, par exemple addto, que l'on utilise rarement dans la mesure où le fichier plain.mp (chargé par défaut au lancement de METAPOST) comprend certaines commandes, draw et fill par exemple, qui sont en fait des macros définies à partir de cette primitive.

Le tableau de la page suivante contient des primitives et des macros définies dans le fichier plain.mp, macros jouant le rôle de commandes (d'où leur présence dans cette liste).

Pour le débogage, il a beaucoup d'autres commandes et variables internes disponibles mais, au niveau débutant, on peut se limiter à la commandes show éventuellement accompagnée de l'opérateur known (sect. 4.2); voici trois exemples typiques d'utilisation :

- show a, pa; montre à la console les valeurs du nombre a et de la 
paire pa, ce qui permet de réaliser que l'on a mal expliqué à METAPOST ce que l'on voulait faire;

- show ca intersectiontimes $\mathrm{cb}$; donne $(-1,-1)$ si les chemins $\mathrm{ca}$ et $\mathrm{cb}$ ne se coupent pas;

- show "oui"; et show "non"; insérés respectivement dans chacune des suites d'instructions d'une boucle permettent de découvrir parfois que tout se déroule bien différemment de ce que l'on espérait!

\begin{tabular}{|c|c|}
\hline Commande & Action \\
\hline addto....also... & $\begin{array}{l}\text { primitive pour "assembler " les éléments d'un } \\
\text { dessin }\end{array}$ \\
\hline btex...etex & $\begin{array}{l}\text { entourent le matériel que } \mathrm{T}_{\mathrm{E}} \mathrm{X} \text { doit composer préala- } \\
\text { blement }\end{array}$ \\
\hline clip & $\begin{array}{l}\text { découpe la partie de la figure courante intérieure à } \\
\text { un contour }\end{array}$ \\
\hline draw & trace un chemin ou un dessin \\
\hline drawarrow & trace un chemin terminé par une flèche \\
\hline fill & mplit un contour fermé \\
\hline filldraw & $\begin{array}{l}\text { trace et remplit un contour fermé (usage délicat, } \\
\text { macro associée: unfilldraw) }\end{array}$ \\
\hline newi & définit une nouvelle variable interne \\
\hline save & $\begin{array}{l}\text { sauvegarde la valeur d'une variable au début de } \\
\text { l'exécution d'une macro et redonne cette valeur à la } \\
\text { fin de l'exécution si la macro est limitée par une paire } \\
\text { begingroup...endgroup }\end{array}$ \\
\hline show & $\begin{array}{l}\text { montre à la console les expressions (valeur ou code } \\
\text { correspondant, cf. section 4.2) }\end{array}$ \\
\hline str & $\begin{array}{l}\text { transforme un suffixe en une chaîne de caractères } \\
\text { contenant les mêmes caractères }\end{array}$ \\
\hline & efface un chemin ou un dessin \\
\hline & \\
\hline verbatir & nplace etex pour passer des commandes à $\mathrm{T}_{\mathrm{E}} \mathrm{X}$ \\
\hline
\end{tabular}

\subsection{MACROS DE BASE}

Le tableau du haut de la page suivante donne les macros de base [121122] les plus utilisées par le débutant et définies dans le fichier plain.mp. Il faut noter que le tableau cité contient en plus les macros de base destinées à la construction de boîtes et définies dans le fichier boxes . mp.

Cahiers GUTenberg no 52-53-Octobre 2009 


\subsection{Construction de macros}

On a déjà vu les cas simples de macros sans paramètre et de macros avec paramètres du type expr qui signifie que ces paramètres sont des variables des différents types (sect. 2.3.3).

On revient sur les macros avec paramètre du type text; chaque paramètre de ce type doit être limité par un couple de parenthèses car il peut contenir des virgules (qui ne peuvent plus alors jouer le rôle de séparateur).

\begin{tabular}{|c|c|}
\hline Commande & Action \\
\hline bbox & donne le c \\
\hline beginfig & rque le début d'une figure \\
\hline buildcycle & construit un contour fermé avec des chemins concourants \\
\hline dashpattern & crée un motif de traitillé \\
\hline dotlabel & trace un point et place le label correspondant \\
\hline en & marq \\
\hline in & incrémente une variable nombre (decr fait l'inverse) \\
\hline lak & exécute le tracé de son argument (voir pp. 12 et 76) \\
\hline $\max$ & $\begin{array}{l}\text { donne le maximum d'une suite de nombres (min donne le } \\
\text { minimum) }\end{array}$ \\
\hline thelal & crée un dessin comme label mais ne le trace pas; draw \\
\hline & peut ensuite le tracer avec des options appropriés (transfor- \\
\hline & d'en obtenir le dessin \\
\hline & $\begin{array}{l}\text { tantome (au sens de } \mathrm{I}_{\mathrm{E}} \mathrm{X} \text { ) correspondant (vorr pp. } 6 \text {, } \\
\text { 83) }\end{array}$ \\
\hline
\end{tabular}

Ces arguments peuvent être une suite d'instructions incluant des macros avec paramètres fixés (macro axespapiermilli, sect. 2.3.4) ; ils peuvent être aussi une liste de caractères qui devient un nom de variable lors de l'exécution (macro arcd, sect. 3.5.1) ; ils peuvent être une liste de suffixes séparés par des virgules utilisée pour l'incrémentation dans une boucle, macro beginbox dans le fichier boxes .mp.

Dans le cas de la macro arcd, l'argument du type text est d'un type particulier car il devient le nom d'un chemin; il ne contient ni virgule ni point-virgule : pour un tel paramètre il a été introduit le type de paramètre suffix [84] moins général que le type text mais plus facile à utiliser. La macro arcd peut fonctionner en prenant un argument du 
type suffix au lieu du type text. Ce type d'argument est utilisé par les macros vardef examinées au paragraphe suivant.

Les macros du type vardef [85] sont ainsi nommées parce que, dans leur définition, def est remplacé par vardef. On peut dire que ces macros sont des «macros-tableaux » où l'indice du tableau est un suffixe ou bien des «macros-variables" dont la variable est un suffixe; c'est le cas de boxit, circleit, diamit, etc. (sect. 5.2) et donnees, tracebf, tracebe, etc. (sect. 5.3).

Il y a enfin les macros primaires, secondaires et tertiaires [88-89] dont le but est essentiellement de permettre l'utilisation de paramètres sans délimiteurs ${ }^{5}$ : les paramètres des types expr et suffix sont placés entre parenthèses et séparés par des virgules; ceux du type text sont individuellement placés entre parenthèses; mais on peut aussi imaginer d'autres définitions ayant d'autres syntaxes d'utilisation. Ce type de macro sort largement du cadre de ce petit manuel (ainsi que certains aspects des macros vardef) : on va simplement «illustrer » la motivation avec un exemple. Imaginons que l'on définisse le produit spécial $a * b-$ $(a+b)$ où $a$ et $b$ sont des nombres et pour lequel on veut que la syntaxe d'utilisation soit du type de celle de la multiplication usuelle, c'est-à-dire a prodspe $b$; au lieu de prodspe $(a, b)$;

METAFONT prévoit pour ce type de définition

secondarydef a prodspe $\mathrm{b}=\mathrm{a} * \mathrm{~b}-(\mathrm{a}+\mathrm{b})$ enddef;

en remplacement de

def prodspe $(\operatorname{expr} a, b)=a * b-(a+b)$ enddef;

Le résultat est le même excepté que, dans l'ordre des opérations, les définitions primaires, secondaires et tertiaires sont exécutées dans le même ordre que exponentiation, multiplication et addition.

5. Le lecteur intéressé est invité à consulter l'index du METAFONTBook pour retrouver tous les passages concernant ces types de macros.

Cahiers GUTenberg no 52-53-Octobre 2009 


\section{BOUCLES ET TESTS}

Ces instructions existent dans la plupart des langages; on en donne simplement la syntaxe METAPOST.

\subsection{BOUCLES}

Les instructions de boucles sont [91-92] [M 172] :

- sil'index est un nombre :

for $i=a$ step $s$ until $b$ : (instructions) endfor ;

Dans le cas où le pas d'incrémentation est 1 ou -1 , le début de l'instruction peut être abrégée en :

for $i=a$ upto $b$ : ou for $i=a$ downto $b$ :

- si l'index est un nombre dont les valeurs ne peuvent pas être engendrées par une incrémentation d'un pas donné, on écrit le début de l'instruction :

for $i=1.05,1.58,3.07 \ldots 28.23$ :

c'est-à-dire l'on donne la liste des valeurs de l'indice séparées par des virgules ;

- si l'index est un est un suffixe, le début de l'instruction s'écrit alors :

forsuffixes $i=\operatorname{sufa}$, sufb, sufc ... sufn :

- pour une boucle qui se répète sans fin, la syntaxe est :

forever : (instructions) endfor ;

Pour les sorties conditionnelles de boucle, on a les instructions [93] : exitif (condition) ; ou exitunless (condition) ;

Dans le premier cas, la boucle s'arrête lorsque la condition devient vérifiée (autrement dit lorsque la variable booléenne exprimant la condition prend la valeur true); dans le deuxième cas, la boucle s'arrête lorsque la condition cesse d'être vérifiée (donc lorsque la booléenne prend la valeur false); il est sage de toujours s'assurer que toute boucle forever contient une sortie conditionnelle convenablement écrite; on peut tester le fonctionnement en écrivant :

$\mathrm{a}:=1$; forever: $\mathrm{a}:=\mathrm{a}+1$; endfor ; (arrêter avec Ctrl C!), puis tester la commande d'arrêt de la boucle en écrivant :

$a:=1$; forever: $a:=a+1$; exitif $a>20$; endfor; 


\subsection{Tests}

La syntaxe de l'instruction de test va rappeler quelque chose aux utilisateurs de $\mathrm{T}_{\mathrm{E}} \mathrm{X}$ [80], [M 169] :

- if (booléenne) : (instructions A) else : (instructions B) fi

où les instructions $\mathrm{A}$ (resp. B) sont exécutées si la booléenne a la valeur true (resp. false). On a donné des exemples à la section 4.2.7.

Les tests du type :

if (a) : (A) else: if (b) : (B) else: (C) fi fi

peuvent être abrégés en :

if (a) : (A) elseif (b) : (B) else: (C) fi

Cahiers GUTenberg no 52-53 - Octobre 2009 


\section{CHAPITRE 5 \\ Boîtes et liaisons : organigrammes et algorithmes}

Dans ce chapitre, on va construire des boîtes de différentes présentations (formes, contours, fonds, et positionnement). Toujours pour simplifier, on ne va considérer que des boîtes dont le contenu est un texte de quelques lettres composé avec la fonte phvr8r utilisée pour les exemples de labels (cf. sect. 1.3) ; cela permet encore de voir le résultat des exercices tout de suite à l'écran. Au lecteur d'imaginer que le contenu de la boîte pourrait être un \parbox composé à l'aide de $\mathrm{LTT}_{\mathrm{E}} \mathrm{X}$ ou une figure déjà produite d'une toute autre manière! Pour disposer des commandes nécessaires, il faut charger le fichier rboxes.mp qui appelle le fichier boxes.mp et ajoute une commande pour construire des boîtes rectangulaires avec les angles arrondis.

On reprend le préambule des quatre premiers fichiers de figures auquel on ajoute les commandes

input rboxes.mp

input boitesup.mp

linejoin:=mitered; (flèches pointues, cf. sect. 2.2.4)

ahlength: $=2 \mathrm{pt}$;

rbox_radius : $=3 \mathrm{pt}$; bboxmargin: $=0.5 \mathrm{pt}$; (pour les boîtes)

afin de constituer le préambule du nouveau fichier de figures fige.mp. Quant au fichier boitesup.mp, il permet de faire des boîtes en forme d'ellipse et de losange; il contient aussi quelques macros élémentaires permettant le tracé de boîtes «à la chaîne ", macros aisément modifiables et adaptables par le lecteur de ce document; enfin, il propose quelques autres macros destinées à faire des liaisons entre les boîtes. Bien entendu, ce petit nombre de macros élémentaires écrites par l'auteur de ce petit document ne peuvent rivaliser avec celles des nombreuses extensions spécialisées telles que metaobj par exemple.

METAPOST raconté aux piétons, chap. 5 


\section{Boîtes Rectangulaires}

On va exposer en détail la construction des boîtes de forme rectangulaire car tout ce qui va être explicité est encore valable pour les boîtes de forme différente : les principes de base restent les mêmes, certaines macros ont des noms différents pour les différentes formes mais il y en a d'autres qui gardent le même nom. La construction d'une telle boîte se fait en réalité en deux étapes qui vont être détaillées.

\subsection{Création de la bô̂te}

La création d'une boîte rectangulaire avec la commande [95] boxit.bb(mat);

où bb désigne le nom de la boîte, mat désigne la contenu de la boîte (ce qui est nommé «matériel» dans le langage du TEXBook) : un ou plusieurs mots, une boîte $\mathrm{T}_{\mathrm{E}} \mathrm{X}$ ou $\mathrm{LAT}_{\mathrm{E}} \mathrm{X}$ ou encore une figure déjà faite. Cette création comprend :

- les déclarations des variables du type paire :

bb.c : position du centre de la boîte, $\mathrm{bb} . \mathrm{n}$ : position du milieu de l'arrête supérieure, puis les positions des milieux des trois autres arrêtes avec les suffixes respectifs e, $\mathrm{s}$ et $\mathrm{w}$, bb.ne : position de l'angle supérieur droit de la boîte, puis les positions des trois autres angles avec les suffixes respectifs se, sw et nw, $\mathrm{bb}$. off : déplacement de la boîte par rapport à sa position d'origine;

- les déclarations des variables du type nombre: $\mathrm{bb} . \mathrm{dx}$ : distance entre le matériel et les arrêtes gauche et droite, $\mathrm{bb}$. dy : distance entre le matériel et les arrêtes supérieure et inférieure.

\subsection{TRACÉ DE LA BoÎTE}

Le tracé est assuré par la commande [95] drawboxed (bb) ;

D'abord elle résout les équations reliant les variables déclarées par la commande boxit.bb en prenant par défaut [95] : $b b$ off $=(0,0) ; b b \cdot d x=d e f a u l t d x ; b b \cdot d y=d e f a u l t d y$; Ensuite, cette commande trace la boîte en tenant compte des valeurs calculées des variables. Elle peut tracer plusieurs boîtes dont la liste des noms séparés par des virgules est donnée en argument.

On peut imposer des valeurs à toutes ces variables, mais pas à toutes à la fois! Il faut que le système d'équations linéaires reliant ces variables 
soit résoluble : on ne peut pas, par exemple, donner à la fois n'importe quelle valeur à bb. of $f, b b$. $n$ et bb. dy car on $a$ :

ypart bb.off + (hauteur du contenu) $+\mathrm{bb}$.dy = ypart bb.n;

comme on peut le vérifier sur la figure suivante. Si l'on fait une telle erreur, alors METAPOST affiche à la console le message d'erreur :

«inconsistent equations».

Par contre on peut faire tout ce qui est raisonnable au sens de la résolution des systèmes linéaires : on va voir quelques-unes des possibilités sur la figure 1.

- (BB) est une boîte tracée avec les valeurs par défaut données cidessus : la boîte de matériel est placée avec l'angle bas gauche du B situé à l'origine. Le montant du B ne touche pas l'axe des $y$, il en est séparé par une distance appelée approche gauche qui est une caractéristique spécifique de chaque lettre; on voit que le e de la boîte (es) a une approche gauche beaucoup plus petite (presque nulle, ce qui est logique pour une minuscule galbée à gauche) ; on voit également que le e descend légèrement en dessous de la ligne de base, ce qui constitue une autre caractéristique des lettres galbées à la partie inférieure ; ces remarques étant faites, on ne reviendra pas sur les propriétés des caractères).

\beginfig(1); axespapiermilli $(-2.5,-3.5,35.5,33.5)$;

boxit.bba ("BB"); drawboxed (bba); boxit.bbb ("Bp");

Figure 1

bbb. off $=(13 u, 0)$; drawboxed (bbb); boxit.bbc ("es");

bbc. of $f=(25 \mathrm{u}, 0)$; drawboxed (bbc); boxit.bbd ("ma");

bbd. $c=(7 \mathrm{u}, 13 \mathrm{u})$; drawboxed $(\mathrm{bbd})$;

boxit.bbe("lq"); ypart bbe. of $f=10 u$;

xpart bbe.w=xpart bbc.w; drawboxed (bbe);

boxit.bbf ("to"); bbf .dx=0.1pt;bb.dy=10pt;

bbf . off $=(0,23 \mathrm{u})$; drawboxed (bbf);

boxit.bbg ("eu");

ypart bbg.n-ypart bbg.s=15mm;

xpart bbg.e-xpart bbg. $\mathrm{w}=10 \mathrm{~mm}$;

bbg. of $f=(12 u, 23 u)$; drawboxed (bbg);

boxit.bbh("dp");

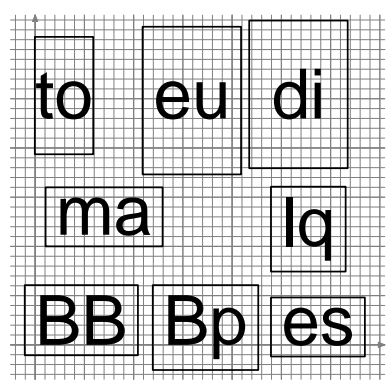

ypart bbh.n-ypart bbh.s=15mm;

xpart bbh.e-xpart bbh.w=10mm;

bbh. off $=(24 u, 23 u)$; drawboxed (bbh);

endfig;

- $(B p)$ est déplacée avec $b b b$. of $f=(13 u, 0)$; (la vérification est aisée grâce à la grille millimétrique) ; il est également important de constater 
que la boîte de matériel était initialement placée à l'origine avec la ligne de base portée par l'axe des $x$ : le déplacement suivant les $y$ étant nul, la ligne de base est toujours portée pas l'axe des $x$.

- (es) est déplacée avec $b b c$. of $f=(25 u, 0)$;

- (ma) est déplacée en écrivant bbd. $c=(7 u, 13 u)$; afin qu'elle soit centrée au point $(7 \mathrm{u}, 13 \mathrm{u})$.

- (Iq) est déplacée avec ypart bbd.off $=10 u$; qui élève de 10u la ligne de base et bbd.w=bbc.w; qui aligne verticalement par les côtés gauches cette boîte avec la boîte (es).

- (to) est une boîte où l'on a fortement réduit la distance séparant le matériel des arrêtes droites droite et gauche et fortement augmenté la distance entre le matériel et les arrêtes supérieure et inférieure; elle est ensuite déplacée vers le haut pour ne pas recouvrir les autres.

- (eu) et (di) sont des boîtes dont on a imposé la hauteur et la largeur en écrivant des égalités du type ypart bb.w-ypart bb.e=15u; Elles ont même ligne de base mais sont décalées l'une par rapport à l'autre car, au sens de $\mathrm{TE}_{\mathrm{E}}$, elles ont une même profondeur (nulle) mais une hauteur différente; ce problème est automatiquement résolu lorsque les boîtes initiales sont des boîtes $\mathrm{T}_{\mathrm{E}} \mathrm{X}-\mathrm{LAT}_{\mathrm{E}} \mathrm{X}$ où l'on rajoute une commande $\backslash$ strut au début et une autre à la fin du texte.

\subsection{AmÉLIORATIONS : FOND ET CONTOUR}

Une fois la macro boxit. bb utilisée on dispose de plusieurs macros qui permettent de diversifier la présentation de ces boîtes : bpath.bb, pic.bb et drawunboxed (bb) permettent de tracer séparément le fond,

Figure 2

fige.mp le contour et le contenu avec des options différentes.

beginfig(2);

axespapiermilli $(-2.5,-2.5,35.5,10.5)$;

defaultscale: $=3$; boxit.bbi ("BB") ;

fill bpath.bbi cl(0.8);

draw bpath.bbi $1 w(1.2 \mathrm{pt})$;

undraw bpath.bbi $I_{w}(0.6)$;

draw pic.bbi cl(0.3);

boxit.bbj ("BB"); bbj. off $=(20 u, 1 u)$;

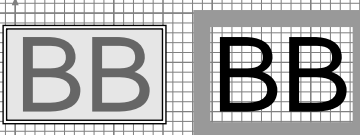

$b b j . d x=d e f a u l t d x+2.5 p t ; b b j . d y=d e f a u l t d y+2.5 p t ;$

draw bpath.bbj lw(5pt) cl(0.6);drawunboxed(bbj);

endfig;

Cahiers GUTenberg no 52-53-Octobre 2009 
Sur la figure précédente on a : un fond gris léger, un contour en trait double et le contenu en gris foncé pour la boîte de gauche et pas de fond, un contour gris très épais et le contenu en noir pour la boîte de droite.

Ces macros disponibles sont les suivantes:

bpath. bb [95] calcule les valeurs des variables comme le fait drawboxed puis devient le nom du contour entourant la boîte,

pic. bb [97] calcule les valeurs des variables comme le fait drawboxed si ce n'est déjà fait puis devient le nom du contenu de la boîte, drawunboxed (bb) [97] calcule les valeurs des variables comme le fait drawboxed si ce n'est déjà fait puis trace le contenu de la boîte en noir (couleur par défaut).

La figure appelle deux remarques importantes.

- La frontière extérieure du contour de la deuxième boîte a les angles vifs : c'est le résultat de la valeur mitered donnée à la variable interne line join dans le préambule du fichier fige .mp afin d'obtenir des flèches pointues. Avec la valeur par défaut rounded, la frontière extérieure du contour aurait des angles arrondis (sect. 2.1 et 2.2.2). Cet effet serait d'autant plus visible que l'épaisseur du trait du contour est forte; il faut bien noter que cet effet n'a rien à voir avec l'arrondissement des angles du contour des boîtes que l'on va voir à la prochaine section (macro rboxit); dans ce cas, ce sera la ligne centrale du contour qui aura les angles arrondis.

- L'utilisation de fortes épaisseurs de trait du contour exige une augmentation des variables $\mathrm{bb} . \mathrm{dx}$ et $\mathrm{bb}$.dy pour garder toujours la même distance entre le matériel contenu dans la boîte et le contour de la boîte (augmentation d'une demi-épaisseur exactement).

\section{AUTRES FORMES : ARRONDIES, CIRCULAIRES, ETC.}

Il y a quatre autres formes possibles pour les boîtes; les commandes précédemment vues, drawboxed(bb), bpath.bb, pic.bb et drawunboxed $(\mathrm{bb})$, sont utilisables et ont toujours le même rôle (bb étant toujours le nom de la boîte). Ces quatre commandes de création de boîtes sont le suivantes.

rboxit.bb crée une boîte avec les angles arrondis, boîte(so), par un quart de cercle de rayon rbox_radius (cette variable interne est réduite dans le préambule du fichier fige.mp de 8 bp à 3 pt pour des raisons d'ordre esthétique). Les huit paires bb.n, bb.s, etc. représentent 
toujours les coordonnées des mêmes points (milieu de l'arrête supérieure, milieu de l'arrête inférieure, etc.

circleit.bb, abrégée en circit.bb [99] crée un boîte de forme circulaire; bb.dx prend la valeur par défaut mais, dans ce cas, bb.dy prend une valeur définie pour que le contour soit un cercle, boîte (eu); on peut cependant fixer bb.dy comme l'on veut et l'on obtient alors une ellipse; c'est en introduisant l'équation $\mathrm{bb} . \mathrm{dx}=\mathrm{bb}$. dy; dans cette macro (et en y faisant quelques aménagements supplémentaires) qu'a été créée la macro suivante donnant un contour elliptique; pour cette forme et pour les autres deux formes possibles, d'une part, les paires correspondant aux directions intermédiaires bb.ne, bb. se, etc. ne sont plus définies et d'autre part, la paire bb.n représente les coordonnées du point le plus haut, la paire bb.s représente les coordonnées du point le plus bas, etc. ellit(bb) crée un boîte elliptique, boîte (as); il faut, pour cette forme de boîte et pour la suivante charger le fichier complémentaire boitesup.mp.

diamit (bb) crée une boîte en forme de losange, boîte (oc); cette macro

Figure 3

fige.mp
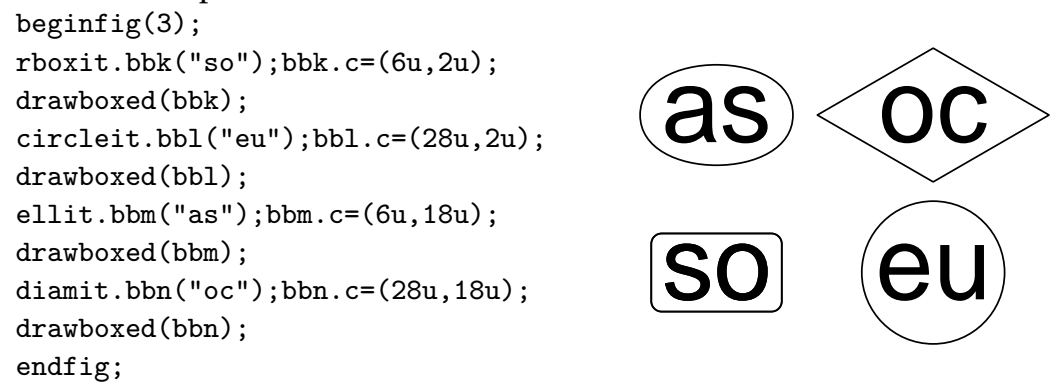

\section{Macros ÉlÉMentaires À LA DeMANde}

On commence par faire le point sur les étapes nécessaires pour la construction des boîtes.

- On dispose de cinq formes prédéfinies pour lesquelles les macros de création correspondantes ont toujours la même syntaxe :

boxit.bb, rboxit.bb, circit.bb (équivalent de circleit.bb), ellit.bb et diamit.bb qui créent les boîtes en prenant le contenu pour argument.

Cahiers GUTenberg no 52-53 - Octobre 2009 
- Après la création, on donne diverses spécifications :

- positionnement; ex: bb.c=(3u, 25u);

- dimensionnement; ex : ypart (bb.n-bb.s) $=16 \mathrm{u}$;

- marges internes; $\mathrm{ex}: \mathrm{bb} . \mathrm{dx}=6 \mathrm{pt}$;

- Ensuite, on dispose de la macro bpath.bb qui est le nom du contour de la boîte; cela permet d'obtenir :

- le tracé d'un fond coloré; ex : fill bpath.bb cl(0.95);

- le tracé du contour; ex: draw bpath.bb lw(0.4pt) cl(0.4);

- la rotation du contour autour de son centre; pour cela il faut introduire, dans les deux commandes ci-dessus et immédiatement après bpath, l'instruction rotatedaround (bb.c,90); pour obtenir par exemple la position «lecture de bas en haut».

— Enfin, avec la macro pic. bb qui désigne le contenu de la boîte, on peut obtenir :

- le tracé du contenu en couleur; ex: draw pic.bb cl(0.4);

- la rotation du contenu autour de son centre ; après avoir tourné le contour et le fond, on place encore la même instruction après pic. bb. Cela paraît un peu fastidieux à utiliser : il n'en est rien car, dans un document, on ne va pas faire des boîtes de toutes les manières possibles pour montrer son savoir, on va choisir un petit nombre de types de boîte bien adaptés aux types de contenu pour faciliter le travail d'assimilation du lecteur.

Pour cela, le fichier boitesup .mp contient une macro de base qui permet à l'utilisateur de construire très facilement des macros donnant des boîtes de différents styles; sept exemples de ces macros sont fournies et seront très certainement suffisantes dans bien des cas. Bien évidemment, ces macros ne prétendent pas rivaliser avec celles des extensions spécialisées.

Cette macro de base est; btrace (pos, ang) (nom) (fond) (contour) (contenu); pos est la paire correspondant au centre de la boîte, ang est l'angle de rotation de la boîte autour du centre, nom est le nom de la boîte, fond est une liste de caractères caractérisant le fond de la boîte, contour est une liste de caractères caractérisant le contour, contenu est une liste de caractères caractérisant le contenu. 
Par exemple, en composant des algorithmes, on veut représenter les données par des boîtes de forme elliptique avec un fond gris léger, un contour en trait double et le texte en noir; pour cela, on définit la macro : vardef donnees@ (pos,ang) =

btrace (pos, ang) (@\#) (ffgl) (ccd) (ddn) enddef;

où @\# est le nom de la boîte, pos et ang sont définis ci-dessus et où les autres trois arguments sont déterminés après avoir consulté le tableau suivant que tout débutant peut compléter tant les macros définissant ces spécifications sont élémentaires (voir le fichier boitesup.mp).

\begin{tabular}{l|l||l|l}
$\mathrm{ffo}$ & pas de fond & $\mathrm{cceg}$ & contour épais gris \\
$\mathrm{ffgl}$ & fond gris léger & $\mathrm{ccd}$ & contour trait double \\
$\mathrm{ffgc}$ & fond gris clair & $\mathrm{ddo}$ & pas de contenu \\
$\mathrm{ffgf}$ & fond gris foncé & $\mathrm{ddn}$ & contenu noir \\
$\mathrm{cco}$ & pas de contour & $\mathrm{cdgc}$ & contenu gris clair \\
$\mathrm{ccfn}$ & contour fin noir & $\mathrm{ddg}$ & contenu gris \\
ccen & contour épais noir & $\mathrm{ddgf}$ & contenu gris foncé
\end{tabular}

On écrit pour chaque boîte de ce type (exemple page suivante) : ellit.bb(Donnees); donnees.bb $((12 u, 42.5 u), 0)$;

Voici deux macros passe-partout caractérisées par : pas de fond, un contour noir fin pour la première (d'où le $f$ final) et épais pour la deuxième (d'où le e final) et le contenu en noir (exemple page suivante) : vardef tracebf@\#(pos, ang) =

btrace (pos, ang) (@\#) (ffo) (ccfn) (ddn) enddef;

vardef tracebe@\#(pos, ang) =

btrace (pos, ang) (@\#) (ffo) (ccen) (ddn) enddef;

On trouvera sur le listing de la figure de la page suivante quatre autres macros définies dans le fichier boitesup.mp; pour faciliter la lecture, le nom des macros de ce type est, excepté pour les deux macros passepartout, constitué par la chaîne constituant le contenu de la boîte.

Les spécifications supplémentaires de dimensions (marges intérieures et dimensions imposées des boîtes) doivent être données, dans le cas de l'exemple ci-dessus, entre la macro ellit.bb et la macro donnees.bb et ce pour chaque boîte. C'est pour faciliter cette situation qu'une deuxième version de la macro de base a été créée (voir encore le fichier boitesup.mp) :

bbtrace (pos, ang) (nom) (spec) (fond) (contour) (contenu);

Cahiers GUTenberg no 52-53-Octobre 2009 
Elle fonctionne de la même manière avec en plus un argument complémentaire spec constitué par des équations imposant des valeurs aux marges intérieures ou aux dimensions des boîtes (sect. 5.1.2). On donne un exemple de l'utilisation de bbtrace pour créer une macro donnant des boîtes de hauteur donnée : vardef rresultats@\#(pos,ang) = bbtrace (pos, ang) (@\#) (ypart (@\#.N-@\#.s)=9u) (ffgl) (ccd) (ddn) enddef ;

beginfig (4);

defaultscale: $=1.2$;

axespapiermilli $(-1.5,-1.5,34.5,47.5)$;

ellit.ba("Donnees");

donnees.ba $((12 \mathrm{u}, 42.5 \mathrm{u}), 0)$;

cirit.bb("Calcul");

tracebe.bb((12u, 29u),0);

diamit.bc("Test");

test.bc $((12 u, 15 u), 0)$;

rboxit.bd("Resultats");

resultats.bd $((12 \mathrm{u}, 4 \mathrm{u}), 0)$;

boxit.be("Retour");

retour.be ( (30u, 33u), 90);

boxit.bf ("Fin");

fin.bf ((30u, 12u), 90);

rboxit.bg ("RResultats");

rresultats.bg $((12 u, 4 u), 0)$;

endfig;

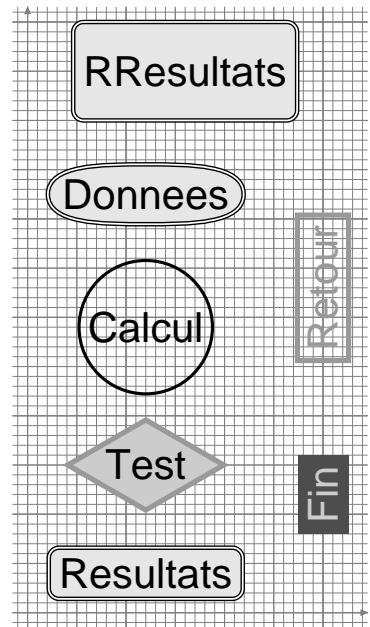

Figure 4

fige.mp

\section{LIAISONS ENTRE LES BOÎTES}

4.1. EXTRÉMitÉS DES LIAISONS

Il y a deux possibilités pour les extrémités des liaisons; on peut choisir :

— le centre des boîtes,

- un des quatre points principaux définis sur le contour des boîtes : ce sont ces points qui vont être utilisés par la suite pour les raisons qui sont mises en évidence sur l'exemple suivant.

Une liaison (ou un lien ou encore une flèche) partant du centre de la boîte bh et allant au centre de la boîte bi s'obtient avec :

drawarrow bh.c -- bi.c

cutbefore bpath.bh cutafter bpath.bi cl(1pt); 
Cette méthode (flèche 1, exemple page suivante) a un premier inconvénient : les opérateurs cutbef ore et cutaf ter suppriment bien les parties de la liaison internes aux boîtes mais on ne peut empêcher la flèche de traverser le contour de la boîte cible. La commande :

drawarrow bi.e -- bj.w cl(1pt);

a le même défaut (flèche 2) mais il est facile d'apporter une correction (flèche 3) en écrivant :

drawarrow bk.e -- bl.w+1.5*left cl(1pt);

On a ici utilisé une des très utiles définitions :

up $=(0,1)$, right $=(1,0)$, down $=(0,-1)$ et left $=(-1,0)$

L'exemple montre bien que la correction de positionnement des flèches est satisfaisante : coefficient de 1.5 pour les boîtes avec contour épais ( 1 pt) avec liaisons de même épaisseur (flèche 3 ), coefficient 0.75 pour les boîtes à contour fin (0.6 pt) avec liaisons de même épaisseur (flèche 5) et coefficient 1 pour les boîtes à contour épais avec liaisons fines (flèche 4). En outre, on constate que la méthode se prête fort bien pour traiter les cas où plusieurs liaisons partent de (ou arrivent sur) la même boîte (flèches 3, 4 et 5). Pour faciliter la lecture de la fin de ce chapitre, le nom

Figure 5 fige.mp des boîtes est rappelé dans leur contenu.

beginfig(5);

defaultscale:=1.3;

rboxit.bh("bh"); tracebe.bh $((5 \mathrm{u}, 0), 0)$;

rboxit.bi("bi"); tracebe.bi ((30u,0),0);

rboxit.bj("bj"); tracebe.bj $((5 \mathrm{u}, 10 \mathrm{u}), 0)$;

rboxit.bk ("bk"); tracebe.bk ((30u,10u),0);

rboxit.bl("bl"); tracebf.bl ((5u,20u),0);

rboxit.bm("bm"); tracebf.bm ((30u, 20u), 0);

drawarrow bh.c--bi.c cutbefore bpath.bh

cutafter bpath.bi lw(1pt);

drawarrow bj.e--bk.w lw(1pt);

drawarrow bj.e+3*up--bk.w+3*up+1.5*left $1 \mathrm{w}(1 \mathrm{pt})$;

drawarrow bj.e-3*up--bk.w-3*up $+1 *$ left $1 \mathrm{w}(0.6 \mathrm{pt})$;

drawarrow bl.e--bm.w+0.7*left $l_{w}(0.6 \mathrm{pt})$;

endfig;

\subsection{LIAISONS À UN SEUL SEGMENT}

La simplicité des commandes ci-dessus permet de définir la macro suivante très simple, facile à utiliser et reportée dans le fichier boitesup.mp :
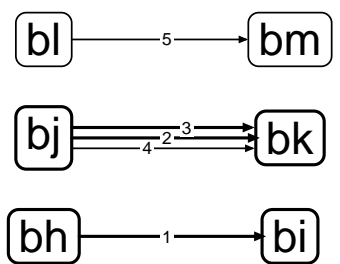

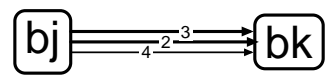

Cahiers GUTenberg no 52-53 - Octobre 2009 
lien (dep, arr) (type);

où dep est la paire représentant le point de départ, arr est la paire représentant le point d'arrivée et type représente le type de lien voulu choisi sur le tableau à double entrée suivant :

\begin{tabular}{l|l|l} 
épaisseur des contours et des liens & trait & flèche \\
contours et lien épais & te & fe \\
contours et liens fins & tf & ff \\
contours épais et liens fins & tm & fm
\end{tabular}

La figure suivante présente un exemple des six possibilités. beginfig(6);

defaultscale: $=1.3$;

Figure 6

rboxit.bop ("bop") ; tracebe.bop $((5 \mathrm{u}, 0), 0)$;

rboxit.boq ("boq"); tracebe.boq $((30 u, 0), 0)$;

rboxit.bor ("bor"); tracebf .bor $((5 u, 10 u), 0)$;

rboxit.bos ("bos"); tracebf .bos $((30 u, 10 u), 0)$;

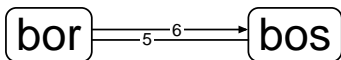

lien (bop.e-4.5*up, boq. w-4.5*up) (tm);

lien (bop.e-1.5*up, boq.w-1.5*up) (fm);

lien (bop.e+1. $5 *$ up, boq. $w+1.5 * u p$ ) (te);

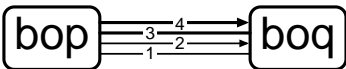

lien (bop.e+4.5*up, boq. $w+4.5 * u p)(f e)$;

lien (bor.e-1.5*up, bos.w-1.5*up) (tf);

lien (bor. e+1. $5 *$ up, bos. $w+1.5 * u p)(f f)$;

endfig;

\subsection{LIAISONS À DEUX SEGMENTS}

En utilisant le calcul de l'intersection de deux droites vu à la section 2.3.2, il est facile d'écrire par exemple la commande qui trace la flèche 3 de l'exemple suivant (sans correction de positionnement mais avec correction de flèche) :

- la première droite est définie par les paires

$z a=b o v \cdot$. e et $z b=b o v \cdot e+p o l a r(1,0)$,

- la deuxième droite est définie par les paires

$\mathrm{zc}=$ bow. $\mathrm{s}$ et $\mathrm{zd}$ =bow $. \mathrm{s}+$ polar $(1,90)$,

- le point d'intersection est définit par

zint=whatever $[z a, z b]=$ whatever $[z c, z d]$,

— le tracé de la flèche se fait alors avec drawarrow za -- zc+1.5*down 1 w (1pt)

Il est encore facile d'écrire une macro relativement simple, facile à utiliser et également reportée dans la fichier boitesup.mp :

llien (dep, angdep, arr, angarr) (type) :

METAPOST raconté aux piétons, chap. 5 
où angdep et angarr sont respectivement les directions orientées au départ et à l'arrivée; les autres notations sont définies pour la macro lien

Figure 7 fige.mp précédente. La figure de la page suivante présente les six possibilités.

\section{beginfig ( 7$)$;}

defaultscale:=1.7; rboxit.bov("bov"); tracebe.bov $((5 u, 0), 0)$;

defaultscale:=1.2; rboxit. bow ("bow"); tracebe.bow $((30 u, 15 u), 0)$;

defaultscale:=1.3; rboxit.box ("box");

tracebf.box $((-2 \mathrm{u}, 8 \mathrm{u}), 0)$; rboxit.boy ("boy")

tracebf .boy $((23 u, 22 u), 0)$;

llien (bov. e-6*up, 0, bow. $\mathrm{s}+6 *$ right, 90$)(\mathrm{tm})$;

llien (bov.e-3*up, 0 , bow. s+3*right,

90) (fm); llien(bov.e,0, bow.s, 90)(te);

llien (bov.e+3*up, 0, bow.s-3*right, 90) (fe);

llien (bow.w, 0, bov.n, -90) (fe);

llien (box.e-1.5*up, 0, boy.s-1.5*left, 90) (tf)

llien (box.e+1.5*up, 0, boy.s+1.5*left, 90) (ff);

llien (boy.w, -180 , box.n, -90 ) (ff);

endfig;

\subsection{LIAISONS À TROIS SEGMENTS ET PLUS}

Au-delà de deux segments, on est obligé de fournir des données supplémentaires; certaines extensions demandent une ou deux longueurs de bras; on choisit la méthode la plus simple : on va se donner un point pour une liaison à trois ou quatre segments et deux points pour une liaison à cinq segments; la liaison complète est ensuite obtenue à l'aide de deux ou trois éléments de base proposés dans les deux sections précédentes. La figure de la page suivante montre un exemple de liaisons à trois, quatre et cinq segments; les points dont les coordonnées ont été données sont repérés par un petit cercle noir et signalés sur le listing par P1, P2, etc.; ils permettent d'identifier facilement les deux ou trois éléments de base constituant la liaison complète; la grille millimétrique aide à bien reconnaître l'effet des lignes du listing. Pour les boîtes en forme de cercle, d'ellipse ou de losange, on remarque que l'on ne peut, avec les deux simples macros (lien et llien), que faire partir (ou arriver) des liaisons aux quatre points principaux bb.n, bb.s, etc.

Cahiers GUTenberg no 52-53-Octobre 2009 
beginfig (8);

Figure 8

axespapiermilli $(-5.5,-5.5,39.5,34.5)$;

fige.mp

$\% \% \% \% \% \% \%$ Fabrication des boîtes

defaultscale: $=2$; ellit.ka("KA") ; tracebe.ka $((5 u, 0), 0)$;

rboxit.kb("kb"); tracebe. $\mathrm{kb}((30 \mathrm{u}, 0), 0)$;

defaultscale:=0.8; cirit.kc("--kc--"); tracebe.kc((5u,15u),0);

diamit.kd("-kd-"); tracebe.kd((30u,15u),0);

defaultscale:=1.2; boxit.ke("--KE--"); tracebe.ke((30u, 30u), 0);

$\% \% \% \% \% \%$ Liens : --P1 (P2--) : lien vers P1 (depuis P2)

lien (ke.s,kd.n) (fe);

lien (ke.s+3*left, (xpart (ke.s+3*left), 21u)) (te); $\quad \%--P 1$

llien ( (xpart (ke.s+3*left), 21u), $-180, k c . n,-90)(f e) ; \quad \%$ P1--

llien (ke.s+6*left, -90 , (xpart ka.w-2u,22.5u),-180) (te); \% --P2

llien ( (xpart ka.w-2u,22.5u),-90,ka.w,0)(fe);

lien (kd.s,kb.n) (fe);

llien (ke.s+3*right, -90 ,

(xpart kd.e+2u,21.75u),0) (te);\%--P3

lien ( (xpart kd.e+2u,21.75u),

(xpart kd.e+2u, 8u)) (te) ;\%P3P4

llien ( (xpart kd.e+2u,8u),

$-180, \mathrm{~kb} . \mathrm{n}+3 * r i g h t,-90)(\mathrm{fe}) ; \% \mathrm{P} 4--$

lien (kc.s,ka.n) (fe);

lien (kd.w, (18.5u,

ypart kd.w)) (te);\% --P5

llien ((18.5u, ypart kd.w),

-90 , ka.e, -180) (fe);\%P5--

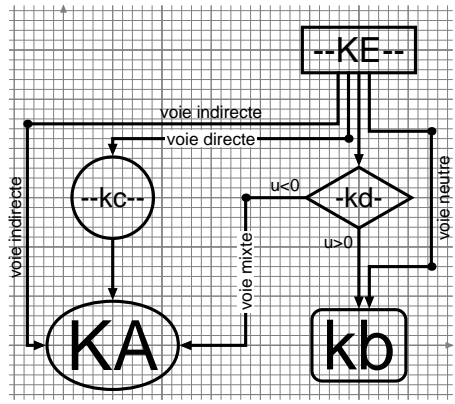

drawpt ( (xpart (ke.s+3*left), 21u)) (2.5pt); \% P1

drawpt ((xpart ka.w-2u,22.5u)) (2.5pt); \% P2

drawpt ((xpart kd.e+2u,21.75u)) (2.5pt); \% P3

drawpt ((xpart kd.e+2u, 8u)) (2.5pt); $\quad \%$ P4

drawpt ((18.5u, ypart kd.w)) (2.5pt); \% P5

$\% \% \% \% \% \% \% \%$ Pose des labels : voir section suivante

defaultscale:=0.5; picture ffa,ffb,ffc,ffd,ffe;

label.top ("voie indirecte", (15u, 22.5u));

ffe=thelabel ("voie directe", (15u,21u));

unfill bbox ffe;draw ffe;

ffa=thelabel.top ("voie indirecte", (xpart ka.w-2u,12u));

draw ffa rotatedabout ( (xpart ka.w-2u, 12u), 90);

label.top ("u<0", (22.5u, 15u)) ; label. left ("u>0", (30u, 10u));

ffc=thelabel.bot ("voie neutre", (xpart kd.e+2u,15u));

draw ffc rotatedaround ( (xpart kd.e+2u,15u),90);

ffd=thelabel ("voie mixte", (18.5u,7.5u));

unfill bbox ffd rotatedaround ( $(18.5 u, 7.5 u), 90)$;

draw ffd rotatedaround $((18.5 \mathrm{u}, 7.5 \mathrm{u}), 90)$;

endfig;

METAPOST raconté aux piétons, chap. 5 


\section{MetTRE DeS LABELS (OU LeTtrageS) SUR LES LiaISONS}

On dispose pour cela des macros suivantes :

- label.xx ("aaa",zz) ;

qui compose la chaîne "aaa" au point de référence $z z$ et la déplace d'une distance labeloff set dans la direction $\mathrm{xx}$ [44] (sect. 1.3) : il faut être attentif à ne pas confondre les quatre directions principales que peut prendre $\mathrm{xx}$ :

top, rt, bot et lft,

et les quatre vecteurs unitaires :

up $=(0,1), \operatorname{right}=(1,0), \operatorname{down}=(0,-1)$ et left $=(-1,0)$.

- thelabel.xx("aaa",zz) : qui crée le dessin contenant la composition de la chaîne "aaa" comme ci-dessus mais ne l'imprime pas [45] : en d'autre termes, si des est une variable dessin déclarée, des=thelabelxx("aaa",zz); draw des; équivaut à label.xx("aaa",zz) ;

On verra ci-après tout l'intérêt de cette macro qui, à première vue, semble inutile. On dispose enfin de la macro :

- bbox des qui donne le chemin rectangulaire entourant le dessin des avec une marge de largeur bboxmargin dans les quatre directions (c'est une BoundingBox agrandie) [50].

Avec ces deux dernières macros, on va pouvoir

— faire tourner le label d'un angle ang autour de son centre en écrivant

draw des rotatedaround (zz, ang);

— faire un fond blanc pour poser le label et le rendre plus lisible en effaçant l'intérieur du chemin rectangulaire entourant le label : on écrit pour cela

unfill bbox des rotatedaround(zz,ang);

où la rotation n'est bien évidemment nécessaire que si le label est tourné lui-même.

Il faut noter que si, par exemple, on veut un label tourné de $90^{\circ}$ et situé à gauche d'une liaison verticale, il faut choisir la valeur top pour xx !

Cahiers GUTenberg no 52-53 - Octobre 2009 
La figure précédente comprend tous les cas possibles :

— ligne (1) : label horizontal en dessus de la liaison (cas le plus simple);

— ligne (2) : label horizontal sur la liaison elle-même;

— lignes (3) et (4) : label vertical à gauche d'une liaison verticale;

— ligne (6) : label horizontal à gauche d'une liaison verticale;

— lignes (9), (10) et (11) : label vertical sur la liaison elle-même;

On reviendra (chap. 7) sur ces macros pour les lettrages composés avec

$\mathrm{T}_{\mathrm{E}} \mathrm{X}-\mathrm{L} \mathrm{T} \mathrm{E} \mathrm{X}$ où l'on rencontrera une petite différence qui facilitera la tâche. 



\section{CHAPITRE 6}

\section{Tracé de courbes}

Dans le cadre d'un petit manuel d'initiation à METAPOST tel que le présent document il est raisonnable qu'après un chapitre consacré aux boîtes et à leurs liaisons il y ait un chapitre concernant le tracé de graphes ou le «tracé de courbes" comme disent les physiciens quand ils reportent sur un document la variation d'une grandeur physique en fonction d'un paramètre; ce langage sera utilisé par la suite.

On va créer un nouveau fichier de figures, figf .mp, en prenant le préambule du fichier fige.mp (avec labeloff set agrandi d'un demi pt) et en y rajoutant :

input graph.mp

qui charge un fichier contenant un ensemble de macros destinées à la présente tâche (ce fichier en charge d'autres, notamment sarith.mp et format.mp qui vont jouer un rôle dans le traitement des grand nombres);

input graphsup.mp

qui appelle un fichier où sont rassemblées quelques macros élémentaires simplifiant l'écriture ou permettant quelques possibilités supplémentaires sans prétention par rapport à des extensions spécialisées; ce fichier commence en donnant la longueur $2 \mathrm{bp}$ (au lieu de $7 \mathrm{bp}$ ) aux repères des graduations des axes, les ticks; ce mot sera utilisé par la suite (cette modification a pour unique but d'avoir une présentation esthétiquement acceptable). On ajoute aussi à ce préambule [161] :

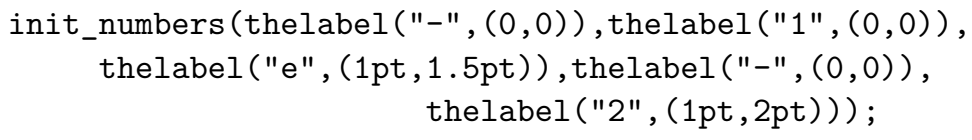

qui est une commande de formatage des valeurs affectées aux graduations pour lesquelles on gardera le mot label; cette commande est nécessaire pour pouvoir voir directement à l'écran le résultat des exercices

METAPOST raconté aux piétons, chap. 6 
sans utiliser TEX-LATEX; bien entendu, elle devra être reformulée différemment pour l'intégration des figures dans un document $\mathrm{T}_{\mathrm{E}} \mathrm{X}-\mathrm{L} \mathrm{T} \mathrm{T} \mathrm{X}$ où l'on voudra alors avoir tous les lettrages composés avec $\mathrm{LAT}_{\mathrm{E}} \mathrm{X}$ (on reviendra donc sur cette commande au chapitre 7).

\section{Tracés PaR défaut}

Les macros puissantes contenues dans le fichier graph . mp permettent de tracer une courbe avec axes et graduations en trois lignes de code

Figure 1 figf.mp

Figure 2 figf.mp comme on le voit sur la figure suivante [141].

beginfig(1);

draw begingraph (43u,30u);

gdraw "data1" 1w(1pt) cl(0.75);

endgraph;

endfig;

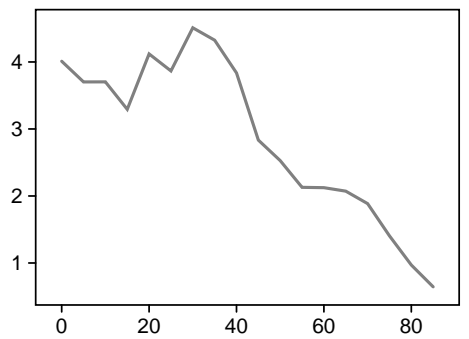

beginfig(2);

path chemin;

draw begingraph $(43 \mathrm{u}, 30 \mathrm{u})$;

chemin $=(0,0)$ for $x=0$ step $10 \% \mathrm{Ex} 1$

until180:.. ( $\mathrm{x}$, sind $\mathrm{x}$ ) endfor; \% Ex 1

$\%$ chemin $=(0,0)$ for $x=0$ step30 \% Ex 2

$\%$ until180:-- ( $x$, sind $x)$ endf or ; $\% \operatorname{Ex} 2$

gdraw chemin ;

endgraph;

endfig;

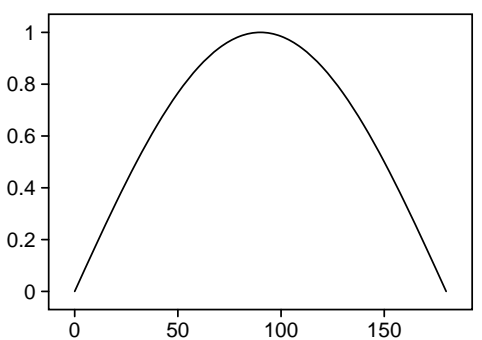

Dans les codes des figures précédentes :

draw begingraph (larg, haut);

est la commande qui débute tous les tracés de courbes : larg et haut sont les dimensions voulues du tracé y compris les graduations (ticks et labels);

endgraph;

est la commande qui termine tous les tracés;

gdraw "data1" ou chemin (options de tracé);

est la commande qui :

- trace la courbe après avoir lu les données dans le fichier data1 ou lu la définition du chemin chemin; ceci après avoir fait les mises à l'échelle

Cahiers GUTenberg no 52-53-Octobre 2009 
nécessaires pour que la figure rentre dans le rectangle de dimensions données et après avoir pris en compte les éventuelles options de tracé;

- construit les axes appropriés avec les ticks et les labels (on note que c'est METAPOST qui choisit les emplacements des graduations).

Les options de tracé [142], situées immédiatement après la chaîne constituée par le nom du fichier (figure 1) ou par le nom du chemin (figure 2), peuvent être : withpen..., withcolor... et/ou dashed... ou bien encore toutes les macros correspondantes $\mathrm{cl}, \mathrm{l}$, etc.

La structure du fichier de données est la suivante : coordonnée $x$, une espace, coordonnée $y$, éventuellement une ou plusieurs espaces et fin de ligne; l'exemple montre comment y insérer des commentaires :

004.011 data1 : nombre d'habitants

053.702 divisé par 1000000

103.703 en fonction de l'âge

......

$85 \quad 0.644$

Bien entendu, le lecteur va vouloir une tout autre présentation du tracé : c'est l'objet de toutes les sections suivantes.

Dans ce chapitre, pour diminuer le nombre de figures, on utilisera la méthode présentée sur la figure 2 ; des parties de code, (Ex 1), (Ex 2), etc., affichent des tracés identiques ou différents suivant les cas. La partie qui n'est pas "cachée " par des \% est celle correspondant à la figure affichée; le lecteur peut ensuite traiter successivement les autres parties par une simple manipulation de \%. Dans la figure qui va suivre, on va introduire encore une autre convention : des parties de code, Tt 1), $\mathrm{Tt}$ 2), etc., peuvent afficher un tracé supplémentaire permettant de faire des comparaisons; pour cela, le lecteur doit simplement enlever les \% «cachant» la (les) partie(s) souhaitée(s).

\section{TYPES DE TRACÉS DISPONIBLES}

Dans la grande majorité des cas, on dispose d'un fichier de données. Le tracé le plus élémentaire est le tracé en "zig-zag" de la figure 1 . Une très minime modification d'une macro du fichier graph .mp permet, moyennant l'utilisation d'une variable interne supplémentaire, d'obtenir un tracé «lissé» de la courbe (fig. 3, page suivante). La macro modifiée et la déclaration de la variable interne nommée lisse sont dans le fichier 
graphsup.mf. La partie de code Tt 1) trace la version zig-zag de la courbe

Figure 3 (figure 1), il permet d'avoir un aperçu de la «valeur» du lissage.

figf.mp
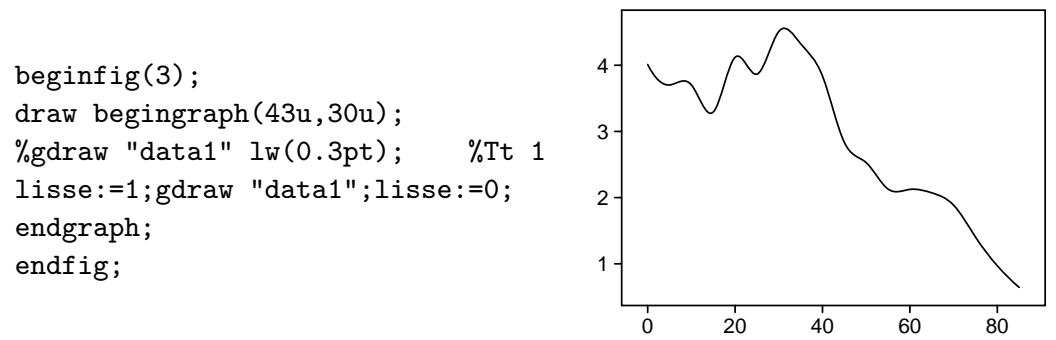

On remarque que le tracé produit à partir du deuxième (resp. premier) chemin de la figure 2 est du même type que le tracé produit avec le fichier de données sur la figure 1 (resp. avec l'option lisse:=1 sur la figure 3) ; il suffit de consulter la macro modifiée Gscan_dans le fichier graphsup.mp pour s'en rendre compte. Par la suite, on ne parlera le plus souvent que de tracés à partir d'un fichier de données.

On peut aussi vouloir marquer les points guides de la courbe par un caractère, un astérisque (figure suivante par exemple), ou tout simplement ne tracer que ces points guides; cela se fait avec l'option de tracé

Figure 4 plot (spécifique à gdraw) [143].

figf.mp beginfig(4);

draw begingraph $(43 \mathrm{u}, 30 \mathrm{u})$; gdraw "data1" Iw(0.3pt) $\%$ Tt 1

$\% 1$ isse:=1; gdraw "data1" cl $(0.7) ; \%$ Tt 1

$\%$ $\%$ Tt 2

\%gdraw "data1" plot lw(0.3pt) ; lisse:=0; \% Tt 2

$\% \operatorname{Ex} 1$

gdata ("data1", s, thelabel $(" * ",(0,0)) ; \% \operatorname{Ex} 1$ glabel ("*",s1,s2) cl(0.3);) \%Ex 2 endgraph;

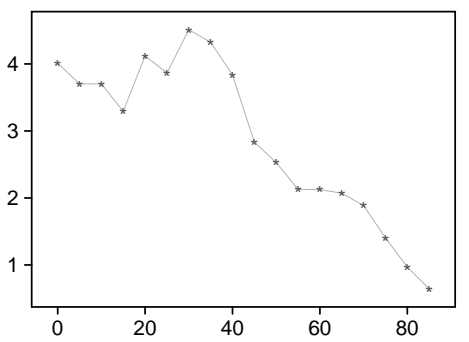

endfig;

Le rôle de plot est le suivant : la macro thelabel a été vue à la en section 5.5 :

draw thelabel ("*" , $(0,0))$; affiche un astérisque à l'origine, draw thelabel $(" * ",(0,0))$ shifted $(a, b)$; affiche un astérisque au point de coordonnées $a$ et $b$.

Ici, dans la figure précédente, partie (Ex 1) :

Cahiers GUTenberg no 52-53 - Octobre 2009 
plot thelabel $(" * ",(0,0))$; affiche un astérisque en tous les points dont les coordonnées sont données dans le fichier lu.

Toujours dans la figure précédente, la partie (Ex 2), on utilise la macro gdata qui donne encore le même résultat avec en plus la possibilité de modifier la couleur de l'astérisque, ce qui n'est pas possible avec la partie (Ex 1). On va expliquer en détail l'utilisation de cette macro dont la syntaxe générale est [152] : gdata ("nom-fichier", s, liste-commandes;) avec les définitions suivantes :

- nom-fichier est le nom d'un fichier de données comprenant $\mathrm{N}$ données par ligne (au lieu de 2) séparées par une espace;

- $\mathrm{s}$ est une variable tableau dont les $\mathrm{N}$ composantes $\mathrm{s} 1, \mathrm{~s} 2 \ldots$ prennent, à la lecture de la ligne $i$ du fichier, les $\mathrm{N}$ valeurs qui s'y trouvent;

- liste-commandes est une liste de commandes exécutées avec l'indice $i$, numéro de la ligne, et les valeurs de $s 1, \mathrm{~s} 2 \ldots$ prises à la lecture de la ligne.

On dispose en outre des macros glabel, gdotlabel, gdraw et gfill qui sont respectivement équivalentes aux macros label, dotlabel, draw et fill excepté que les valeurs données des positions subissent la mise à l'échelle nécessaire pour que la figure ait les dimensions voulues ; on a donc écrit :

gdata ("data1", s, glabel("*", s1, s2) cl(0.5));

On va voir comment ces macros permettent de faire de nombreux types de tracés dont on ne donnera que quelques exemples. La partie (Ex 1) de la figure 5 de la page suivante utilise une bille noire définie préalablement pour montrer que l'on peut utiliser un marqueur faitmaison; ce type de définition, très courant, utilise la commande image [H 11] qui transforme une liste de commandes de tracé en une variable dessin (variable qui peut être tracée par la suite avec toutes les options possibles).

La partie (Ex 2) de cette même figure utilise une possibilité de la macro gdata : elle place un label à chaque point-guide dans la direction appropriée. Les trois premières lignes du fichier de données sont :

004.011 A top

153.293 B bot

304.51

METAPOST raconté aux piétons, chap. 6 
Le test sur la chaîne s3 permet de ne pas mettre de label à tous les points (bien entendu, il faut faire un prétraitement pour déterminer les

Figure 5 figf.mp positions des labels et les reporter dans le fichier de données). $\%<$ def bille=image (draw fullcircle scaled 1.5) enddef; beginfig (5);

draw begingraph (43u, 30u);

string rien; rien=" ";

gdraw "data2" 1w(0.3pt) \%Tt 1 cl $(0.7) ; \%$ Tt 1

$\%$ lisse:=1; gdraw "data2" $\%$ Tt 2 $\% \quad l w(0.3 p t) ;$ lisse: $=0 ; \%$ Tt 2

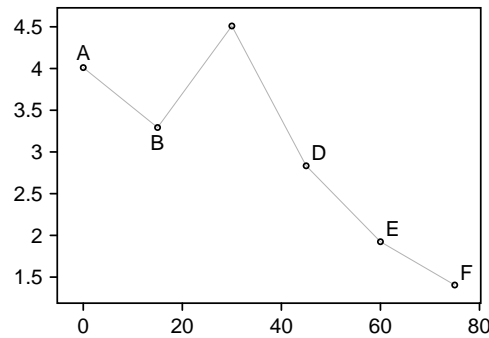

fi; endfor fi;) $\% \operatorname{Ex} 2$

endgraph;

endfig;

La figure 6 de la page suivante utilise la macro augment.p (z) [154] où $p$ est un chemin préalablement déclaré et où $z$ est une paire représentant un point; la première fois que augment est invoquée avec le chemin $p$ et une paire $(a, b)$, elle définit $p$ comme le chemin ponctuel $(a, b)$, la deuxième fois qu'elle est invoquée avec le chemin $p$ et une autre paire $(c, d)$, elle définit $p$ comme étant le chemin $(a, b)--(c, d)$; à chaque lecture de ligne, la macro génère un nouveau segment de la courbe en style zig-zag. Il suffit alors de rajouter la projection de la courbe sur l'axe des $x$ pour obtenir un chemin fermé que l'on colore pour avoir le résultat de la figure. Pour fixer les idées, cette figure représente en gris clair l'énergie consommée par un pays (en unités arbitraires) et en gris foncé la partie de cette énergie d'origine nucléaire ${ }^{6}$. Les colonnes du fichier de données sont les années, la consommation totale (s3) et la partie d'origine nucléaire (s2).

Dans la figure 7 de la page suivante, la macro augment est utilisée, à chaque lecture de ligne, pour construire la «tige de l'épingle " corres-

6. Le lecteur n'aura pas de difficulté pour deviner quel pays a suggéré les données de cette figure.

Cahiers GUTenberg no 52-53-Octobre 2009 
pondante (ce type de tracé est particulièrement apprécié dans certaines disciplines, peut-être avec une tête, macro bille, un peu plus grosse). beginfig(6) ;

draw begingraph (43u,30u);

path $\mathrm{p}, \mathrm{pp}$;

gdata ("data3", s, augment.p (s1, s3) ; )

gfill p-- $(2000,0)--(1950,0)--c y c l e$ cl $(0.85)$;

gdata ("data3", s, augment.pp (s1, s2); )

gfill pp-- $(2000,0)--(1950,0)--$ cycle cl $(0.70)$;

endgraph;

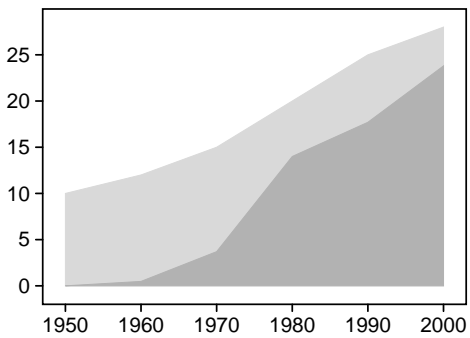

endfig;

beginfig (7);

draw begingraph (43u,30u);

gdata ("data1", s,

glabel (bille, s1,s2);)

gdata("data1", s, path p;

augment.p (s1, s2);

augment.p $(\mathrm{s} 1,0)$; gdraw $\mathrm{p} ;)$

endgraph ;

endfig;

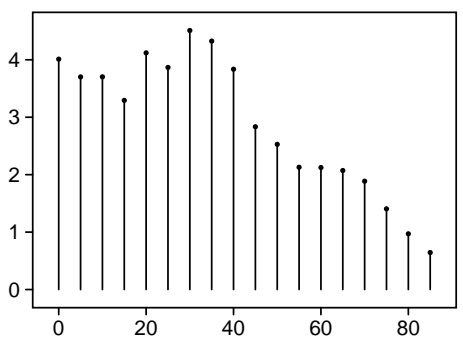

Figure 6

figf.mp

Figure 7

figf.mp

Pour ne pas embrouiller le lecteur, on a gardé, et on gardera autant que possible, les caractéristiques par défaut excepté celles que l'on est en train d'examiner.

\section{DOMAINES DE VARIATION ET TYPES DE COORDONNÉES}

Par défaut, le cadre entourant le tracé est déterminé par les coordonnées de ses sommets inférieur gauche et supérieur droit :

$x_{\min }-a, y_{\min }-b, x_{\max }+a$ et $y_{\max }+b$,

où les coordonnées $x_{\min }, y_{\min }, x_{\max }$ et $y_{\max }$ sont lues dans le fichier de données et où $a$ et $b$ ont des valeurs appropriées pour que ce cadre entoure le tracé à une certaine distance en donnant un résultat visuellement agréable. Si on le veut, on peut modifier cet aspect avec la macro [145] :

setrange (ux, uy, vx, vy);

où ux, uy, vx et vy sont les coordonnées voulues pour les deux sommets cités exprimées avec les mêmes unités que les coordonnées utilisés dans 
le fichier de données (pour supprimer des points, il faut effacer les lignes correspondantes : le fichier ne doit pas avoir de ligne blanche ni de lignes cachées par des \%). Si l'on ne veut modifier que certaines de ces quatre bornes, les autres doivent être affectées avec la chaîne whatever pour prendre leur valeur par défaut déterminée comme expliqué ci-dessus; on donne un exemple assez couramment utilisé :

setrange (origin, whatever, whatever);

où origin est une abréviation de 0,0 .

On est de nouveau confronté au fait que METAPOST n'utilise en interne que des nombres entiers bornés; pour le tracé de courbe, les nombres doivent être inférieurs à 1000 . Pour passer outre cette limitation, il faut écrire les coordonnées données dans la macro setrange sous la forme de chaînes de caractères du type "men" où m est un nombre "acceptable", e est le caractère " $\mathrm{e}$ " et n est la puissance de 10 par laquelle il faut multiplier le nombre précédent pour retrouver le nombre initial trop « grand» [146].

La figure suivante utilise successivement les fichiers data4 et data5 dont les premières et dernières lignes sont les suivantes :

$$
\begin{aligned}
& \text { data4 : } \begin{array}{llllll}
20 & 0.007 & \text { data5 } & 20 & 0.7
\end{array} \\
& \begin{array}{llll}
480 & 279.3 & 480 \quad 27930
\end{array}
\end{aligned}
$$

La partie (Ex 1) utilise le fichier data4 dont les nombres sont acceptables et la macro setrange prend les coordonnées des premier et dernier points. La partie (Ex 2) utilise le fichier data5 où les ordonnées ont été multipliées par 100; mais, le fait d'avoir écrit pour dernier argument de setrange la plus grande ordonnée sous la forme spécifique détaillée cidessus, suffit pour que METAPOST produise le tracé correct qui, bien entendu, coïncide avec le précédent (les labels de l'axe des ordonnées ne

Figure 8 figf.mp sont évidemment pas les mêmes) :
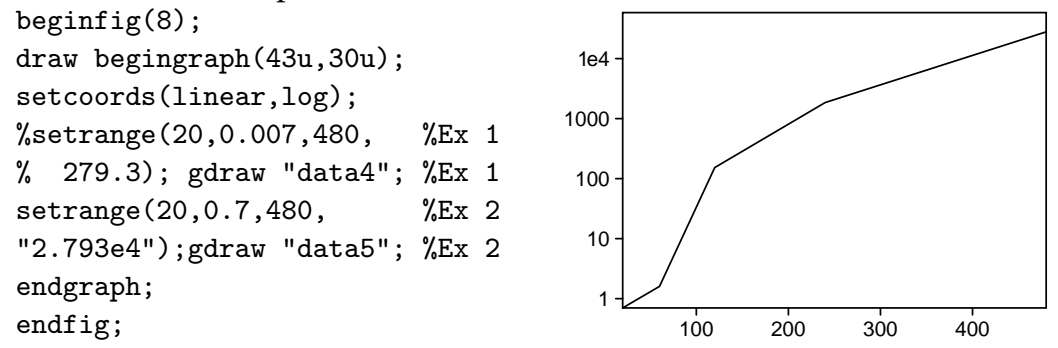
On choisit les types de coordonnées avec la macro [147] : setcoords (typex, typey);

où typex et typey peuvent prendre les valeurs linear, log ainsi que -linear et -log; le défaut correspond linear pour les abscisses et les ordonnées (comme on peut le constater, la figure 8 correspond au choix linear pour les $x$ et log pour les $y$ ). Par défaut, c'est le type linear qui est utilisé pour les abscisses et pour les ordonnées.

On va voir maintenant sur la figure suivante comment on peut tracer, sur une même figure, deux courbes différentes avec des unités différentes suivant les $y$. Sur la figure suivante, on trace, en fonction des années entre 1960 et 2000, l'évolution de la pollution en éléments irritants (exprimée en unités arbitraires, courbe continue) et le pourcentage de personnes ayant nécessité une courte hospitalisation (courbe en traitillé). Ce tracé utilise deux fichiers data 6 et data7, mais on pourrait mettre les données en un seul fichier où les données des deux courbes seraient séparées par un ligne blanche : en arrivant à une ligne blanche, la lecture s'arrête; elle reprend lorsqu'une nouvelle lecture est demandée. Cette propriété fait que tout se bloque si le fichier de données se termine malencontreusement par une ligne blanche, car METAPOST attend une nouvelle commande de lecture du même fichier!

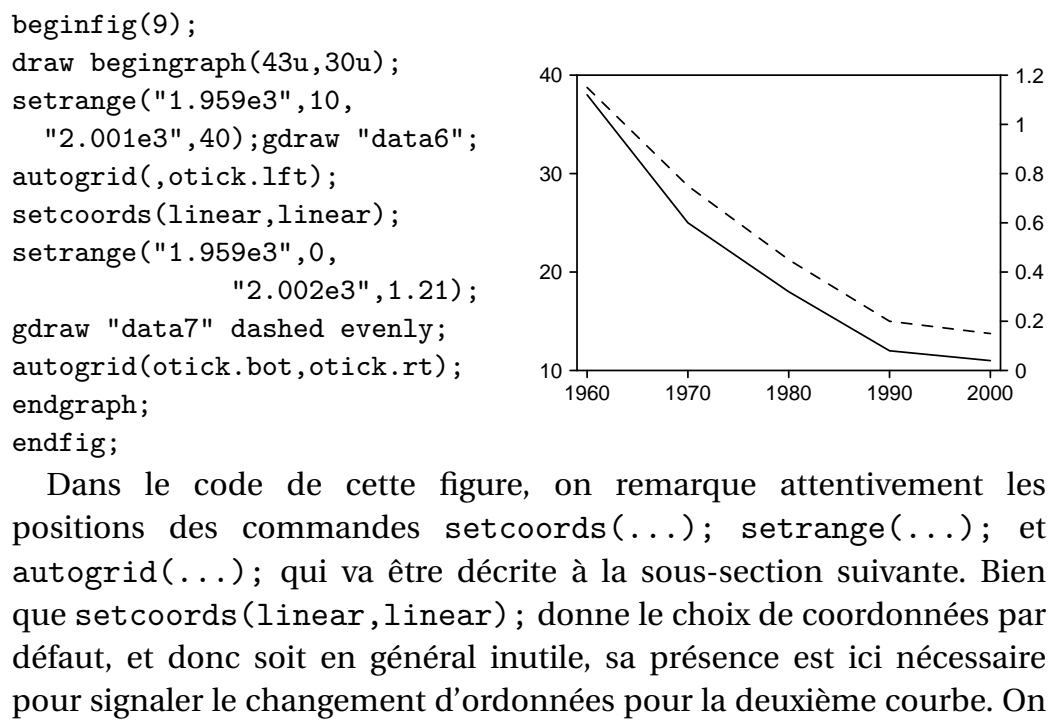

Dans le code de cette figure, on remarque attentivement les positions des commandes setcoords $(\ldots)$; setrange (..); et autogrid(...); qui va être décrite à la sous-section suivante. Bien que setcoords (linear, linear); donne le choix de coordonnées par défaut, et donc soit en général inutile, sa présence est ici nécessaire pour signaler le changement d'ordonnées pour la deuxième courbe. On 
constate aussi que, pour faire apparaître les labels 1960, 2000 et 1.2, on a dû prendre les bornes $1.959 \mathrm{e} 3,2.001 \mathrm{e} 3$ et 1.21 . On verra lus loin comment remédier systématiquement à ces problèmes (liés aux arrondis des calculs internes de METAPOST).

\section{Cadre (OU AXES) ET GRADUations (TICKS ET Labels)}

Les ticks et les grilles sont choisis avec la macro [149] : autogrid (xxx, yyy) (options de tracé);

où xxx pour les abscisses et yyy pour les ordonnées sont soit vides soit prennent les valeurs suivantes:

otick.suff

pour des ticks vers l'extérieur du cadre; suff est un suffixe donnant le côté du cadre où l'on veut placer les ticks : top, rt, bot et lft respectivement pour côté haut, droit, bas et gauche;

itick.suff

pour des ticks vers l'intérieur du cadre; suff joue le même que cidessus;

grid.suff

trace des traits parallèles à partir du côté désigné par le suffixe suff déjà défini ci-dessus; il est recommandé d'ajouter une option de tracé (gris très clair ou couleur claire) pour laisser la ou les courbes bien en évidence;

Enfin si un argument reste vide, alors il n'y a aucune graduation correspondante, c'est le cas de la partie (Ex 1) sur la figure suivante. La partie (Ex 2) montre comment en utilisant deux fois la macro aut of ig, on peut avoir des lignes de grille gris clair pour les abscisses et des ticks noirs

Figure 10

figf.mp pour les ordonnées.

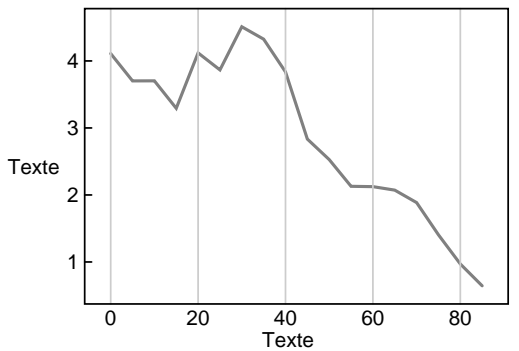

Cahiers GUTenberg no 52-53-Octobre 2009 
Pour modifier le cadre on dispose de la macro [151] : frame.suff (options de tracé);

où suff prend les valeurs top, rt, bot, lft et leurs combinaisons sous une forme spécifique déjà rencontrée $: 1 \mathrm{lft}$ donne les axes traditionnels, partie (Ex 1) sur la figure suivante : en ajoutant frame.rt, partie (Ex 2), on aura un nouvel axe d'ordonnées à droite que l'on peut graduer indépendamment des graduations de l'axe vertical de gauche, comme fait sur la figure 9. Évidemment, on a le cadre complet par défaut. beginfig(11);

draw begingraph $(43 u, 30 u)$;

Figure 11

setrange ( 0 , whatever, whatever, whatever) ;

lisse:=1;gdraw "data1" cl(0.5); lisse:=0;

frame.llft; $\quad \% \operatorname{Ex~} 1$

$\%$ frame.rt; $\quad \% \operatorname{Ex~} 2$

glabel.bot("Age des individus"

, OUT) ;

picture legendey;legendey=thelabel

("Population en millions

d'indivudus", (0.0))

ratated 90; glabel (legendey,OUT);

$\%$ label supplémentaire

$\operatorname{gdraw}(55,2.129)--(70,3) 1 w(0.2 \mathrm{pt})$;

$\operatorname{gdraw}(45,2.834)--(70,3) 1 \mathrm{w}(0.2 \mathrm{pt})$;

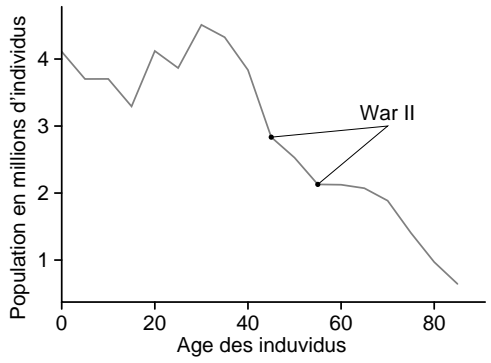

glabel (bille , 55, 2.129); glabel (bille , 45 , 2.834);

glabel.top ("War II",70,3);

endgraph;

endfig;

\section{LÉGENDE DES AXES ET LABELS SUPPLÉMENTAIRES}

Il est important pour le lecteur du document de rappeler sur les figures les grandeurs représentées sur les axes. Pour cela, on dispose de la macro [144] :

glabel.suff ("Texte" , OUT);

où suff est un suffixe qui prend les valeurs déjà rencontrées : bot pour l'axe des abscisses, lft pour l'axe des ordonnées et éventuellement rt pour le deuxième axe de coordonnées; le résultat se trouve sur la figure 10, page précédente.

Pour être parfait, il faut encore faire tourner le texte de la légende de l'axe des ordonnées; comme cela a été fait à la section 5.5, on utilise 
la macro thelabel pour créer une variable dessin nommée legendey située à l'origine et que l'on tourne de 90 degrés; ensuite, la commande glabel (legendey, OUT); trace cette légende; le résultat se trouve sur la figure 11, page précédente.

Il y a des situations où la raison pédagogique nécessite quelques tracés supplémentaires absolument indispensables. On peut mettre en évidence certains points de la courbe par l'ajout d'un astérisque (ou d'un tout autre caractère : une bille sur l'exemple suivant). Cela se fait avec la macro glabel qui permet aussi de placer aussi un petit commentaire. On peut en outre ajouter des traits de "renvoi" afin de relier un commentaire au(x) point(s) concernés. Un exemple de ces possibilités est présenté sur la figure 11 pour signaler un "manque" d'individus ayant entre 45 et 55 ans qui correspond aux naissances pendant les années de guerre et les années d'incertitude précédentes (les données ont été recueillies en 1991).

On aborde enfin, figure 12 de la page suivante, les adaptations possibles concernant les ticks. On peut, d'une manière très simple :

- placer des ticks supplémentaires (macros pxtick et pytick et même remplacer éventuellement les ticks par défaut :

- tracer des traits de renvoi entre les points de la courbe mis en évidence et leurs projections sur les axes. Ces derniers tracés ne peuvent être faits que si les bornes inférieures données en premier et deuxième argument de setrange sont des nombres "acceptables", c'est-à-dire non écrits sous la forme appelée men à la section 6.3.

Il faut remarquer que les définitions pxtick et pytick contiennent respectivement les valeurs particulières à la figure courante $x_{\min }$ et $y_{\min }$ ( 0 et 0 dans le cas de la figure 12). Les labels sont placés tout simplement avec la macro glabel après avoir pris la valeur 4.2 bp pour la distance labeloffset afin que la distance tick-label pour les éléments ajoutés soit identique à cette même distance pour les éléments par défaut. Pour que $v_{1}$ et $v_{2}$ ne se chevauchent pas, on les a rassemblés en un seul label (séparés par une espace) placé entre les ticks correspondants.

Les macros du fichier graph.mp ont des possibilités limitées en contrepartie de leurs avantages dont le principal est bien entendu la mise à l'échelle automatique des données. Cet avantage à un inconvénient: on ne peut pas affiner la position d'un lettrage autrement que par la modification de la distance, comme fait ci-dessus; pour cela il faudrait savoir

Cahiers GUTenberg no 52-53-Octobre 2009 
quel accroissement des $x$ et des $y$ correspond par exemple à un déplacement de 1 pt (METAPOST fait ce calcul en interne).

$\%<$ def trait=image (draw $(0,0.5 \mathrm{bp})--(0,-2 \mathrm{bp})$;

$\%<\quad$ undraw $(0,0.5 \mathrm{bp})--(0,2 \mathrm{bp}))$ enddef;

Figure 12

$\%<$ def pxtick (expr $\mathrm{x}$ ) =glabel (trait, $\mathrm{x}, 0$ ) enddef;

$\%<$ def pytick (expr y) $=$ glabel (trait rotated-90,0,y) enddef;

beginfig(12)

def fonction(expr $\mathrm{x})=1 /(1+(\mathrm{x}-1.5) * * 2)$ enddef;

draw begingraph $(43 \mathrm{u}, 30 \mathrm{u})$;

setrange $(0,0,6.2,1.1)$; path courbe;

courbe $=(0.3$, fonction $(0.3))$

for $i=0.4$ step 0.1 until 6 :

.. (i,fonction(i)) endfor;

gdraw courbe; frame.llft;

autogrid(, );

$a=$ fonction (1); $b=$ fonction (1.3);

glabel (bille,1,a); glabel (bille, 1.3,b);

pxtick(1);pxtick(1.3);

pytick(a);pytick(b);

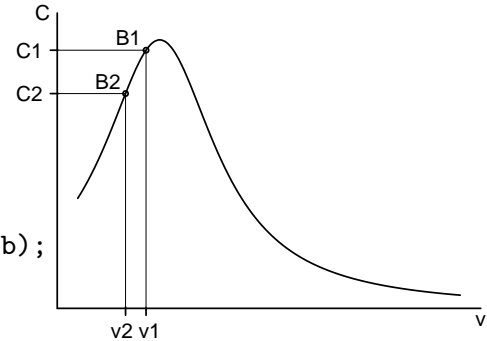

glabel (bille, 1,a); glabel (bille,1.3,b);

gdraw $(1,0)--(1, a)--(0, a) \quad 1 w(0.2 p t)$;

gdraw $(1.3,0)--(1.3, \mathrm{~b})--(0, \mathrm{~b})$ lw $(0.2 \mathrm{pt})$; glabel.lft $(" \mathrm{C} ", 0,1.1)$;

glabel. bot ("v",6.2,0); labeloffset: $=4.2 \mathrm{bp}$;

glabel.bot("v2 v1",1.15,0);

glabel.lft ("C1", 0, b); glabel.Ift ("C2", 0, a) ; labeloff set:=2.2bp;

endgraph;

endfig;

\section{DifFICULTÉS DUES AUX GRANDS NOMBRES}

Des difficultés ont été cachées : c'est le moment d'en parler! On commence avec la figure 9 (où l'on avait dû faire une "réparation " pas très rigoureuse) pour laquelle les années concernées sont en vérité les années 2000 à 2008. Si l'on refait cette figure, figure 13 de la page suivante, avec la modification proposée, alors on constatera, en reprenant la partie (Ex 1) et en cachant la partie (Ex 2), un problème d'affichage des graduations de l'axe des abscisses.

Le problème est résolu en annulant l'affichage des graduations de l'axe des abscisses avec autogrid et en le réalisant directement «à la main » et en dehors de autogrid avec la commande format que l'on va détailler plus loin; il suffit de recacher la partie (Ex 1) et de reprendre la partie 
Figure 13

figf.mp
(Ex 2) pour avoir le résultat [152]. Cette méthode est utilisable chaque fois que les graduations par défaut sont incorrectes où ne sont pas celles que l'on voudrait.
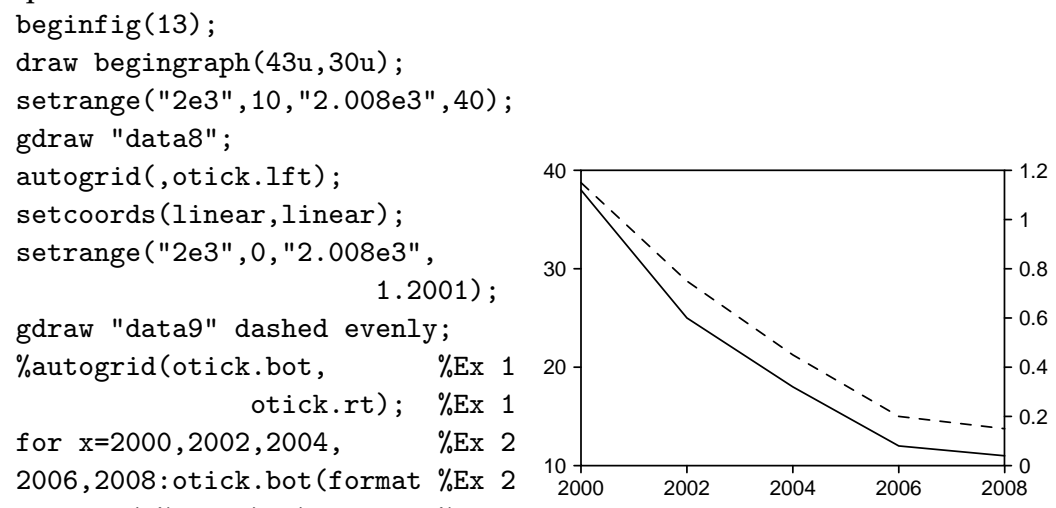

Pour comprendre, il faut approfondir le problème des formats; en réalité, on peut dire qu'il y a deux sortes de formats.

Les formats de composition (ainsi nommés car ils sont utilisés pour l'affichage des labels) sont choisis avec la commande init.numbers [152,161] donnée en tout début de ce chapitre afin de visualiser les figures sans passer par $\mathrm{T}_{\mathrm{E}} \mathrm{X}-\mathrm{LAT}_{\mathrm{E}} \mathrm{X}$ mais en utilisant la fonte PostScipt Helvetica; pour comprendre le rôle des paramètres de cette commande, il suffit d'en considérer un : si on avait écrit thelabel ("2", $(0,0)$ ) au lieu de thelabel ("2", (1pt, 2pt)), les puissances de dix seraient trop près du caractère " $\mathrm{e}$ " et pas assez hautes, voir la figure 8 . C'est ce format qui va changer lorsque l'on intégrera les figures dans un document IATEX, chapitre 7.

Les formats de production (ainsi appelés car ils sont utilisés dans les calculs) sont des chaînes de caractères du type [158] :

"\% $\mathrm{px} "$

— p est un nombre optionnel représentant la précision; par défaut, $p=3$;

- $x$ est une lettre e, $f, g$ ou $G$; pour les choix e et $f$, p est le nombre de chiffres significatifs après arrondi; pour les choix $f$ et $G$, le nombre est 
arrondi au multiple de $10^{p}$ le plus près;

On revient à l'exemple : le format par défaut "\%g" arrondit 2000, $2001, \ldots, 2004$ à 2000 et 2005, 2006,... à 2010, d'où la nécessité d'imposer le format "\% $4 \mathrm{~g} "(p=4)$ pour avoir les labels de l'axe des abscisses corrects.

Il est difficile de prévoir tous les cas qui mériteraient un exemple. On reprend sur la figure suivante une difficulté rencontrée pour la figure 1 : le nombre d'habitants avait été, sur le fichier, divisé par un million, tâche fastidieuse dans les cas de gros fichiers. En cachant la partie (Ex 2) et en prenant la partie (Ex 1), les graduations ont la forme 1e6; cependant il est nettement préférable de revenir a une graduation en millions d'individus. Ce résultat peut s'obtenir directement en reprenant la partie (Ex 2) et en cachant la partie (Ex 1) ; la macro Mreadpath lit le fichier de données et permet de diviser les ordonnées par $10^{6}$ à condition de modifier la variable interne Gpaths :

beginfig(14);

draw begingraph (43u, 30u);

Figure 14

setrange $(0,0,85,4.8 \mathrm{e} 6)$;

\%gdraw "data10"; \% Ex 1:1 ligne

Gpaths:=log; $\quad \%$ Ex 2:3 lignes

gdraw Mreadpath("data10")

shifted (0,-6*Mten); Gpaths:=linear;

glabel (bille, 50, 2.528); glabel

("A" , 50, 2.528); glabel (trait , 50, 0);

glabel (trait rotated $-90,0,2.528$ );

labeloff set : $=4.2 \mathrm{bp}$;

glabel.Ift ("2.52" , 0, 2.528);

glabel.bot (" $50 ", 50,0)$;

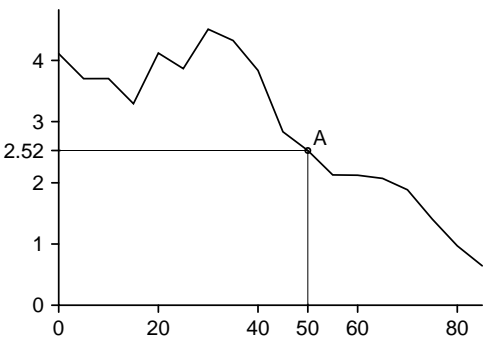

labeloff set: $=2.2 \mathrm{bp} ; \operatorname{gdraw}(-5,2.528)--(50,2.528) l_{\mathrm{w}}(0.3 \mathrm{pt})$;

frame.llft;

endgraph;

endfig;

La macro Mreadpath [156] lit un fichier de coordonnées dont la structure est celle du fichier utilisé pour la figure 1 et donne le chemin correspondant; il faut ensuite préciser comment doivent être traitées les données lues : pour le cas présent, la variable interne Gpaths (valeur par défaut linear) doit avoir la valeur log. Ce chemin est ensuite tracé avec gdraw après avoir subi une translation, mais ici une translation de -6 sur les puissances de 10 (la variable Gpaths a pour valeur log) est bien 
une division par un million! On a mis en évidence un point de la courbe et on a vérifié que l'on pouvait tracer les traits de rappel, mais ceci après avoir modifié la valeur de l'abscisse minimum (cf. la réserve faite à la fin de la section 6.5).

\section{Tracé de COURBeS METAPOST « RELOOKÉES »}

Il est dommage de se priver de la puissance des macros fournies dans le fichier graph.mp, notamment de la machinerie qui fait la mise à l'échelle : on peut imposer des dimensions au tracé sans modifier les données. Non seulement METAPOST fait cette mise à l'échelle mais calcule les valeurs des graduations. On peut exploiter cette puissance et obtenir une présentation plus discrète avec quelques macros (fournies dans le fichier graphsup.mp) exploitant les résultats de calculs faits en interne. Voilà la marche à suivre.

Étape 1 : On fait le tracé de la courbe normalement, c'est-à-dire en cachant les parties de code (Ex 1), (Ex 2) et (Ex 3); on peut utiliser des améliorations à condition de garder le cadre et les graduations par défaut. Dans le cas de la figure 15 de la page suivante (représentation de la courbe sinus hyperbolique), un calcul élémentaire nous suggère de prendre $\left|x_{\min }\right|$ et $\left|x_{\max }\right|$ ne dépassant pas 4 ou 5 pour que $e^{x}$ soit inférieur à 1000) : on choisit 4 . On compile uniquement la partie commune du code et on regarde le résultat à l'écran : compte tenu des graduations affichées, on choisit 32 pour $\left|y_{\min }\right|$ et $\left|y_{\max }\right|$.

Étape 2 : On peut alors ajouter la commande setrange avec les 4 valeurs choisies; cet ajout est facultatif, il a pour but de diminuer l'espace entre la courbe et le cadre; en effet, sans cette commande, la valeur par défaut de cet espace sera trop grande et il y aura trop d'espace entre la figure et sa BoundingBox. Après cette petite amélioration recommandée, on passe à l'opération principale : avec la partie (Ex 3), on supprime le cadre et les graduations par défaut et, avec la partie (Ex 1), on trace de nouveaux axes gradués en reproduisant les valeurs des graduations lues sur le résultat de la première étape. Pour cela on utilise les macros suivantes :

- pmaxes (xmin, ymin, xmax, ymax);

trace les axes.

- pmxticks (xstep, xxmin, xxmax);

trace les graduations de l'axe des abscisses; pour xxmin et xxmax on

Cahiers GUTenberg no 52-53-Octobre 2009 
choisit (toujours d'après la visualisation) un pas de 1 et les deux couples de bornes $-4,1$ et 3,4, pour éviter de tracer la graduation en $x=2$; la raison en est que l'on veut éviter un recouvrement de lettrages par la suite. Le tick et le label en $x=0$ ne sont pas tracés; si nécessaire, on rajoute le label «à la main » avec glabel, en général dans le cadran bas droit.

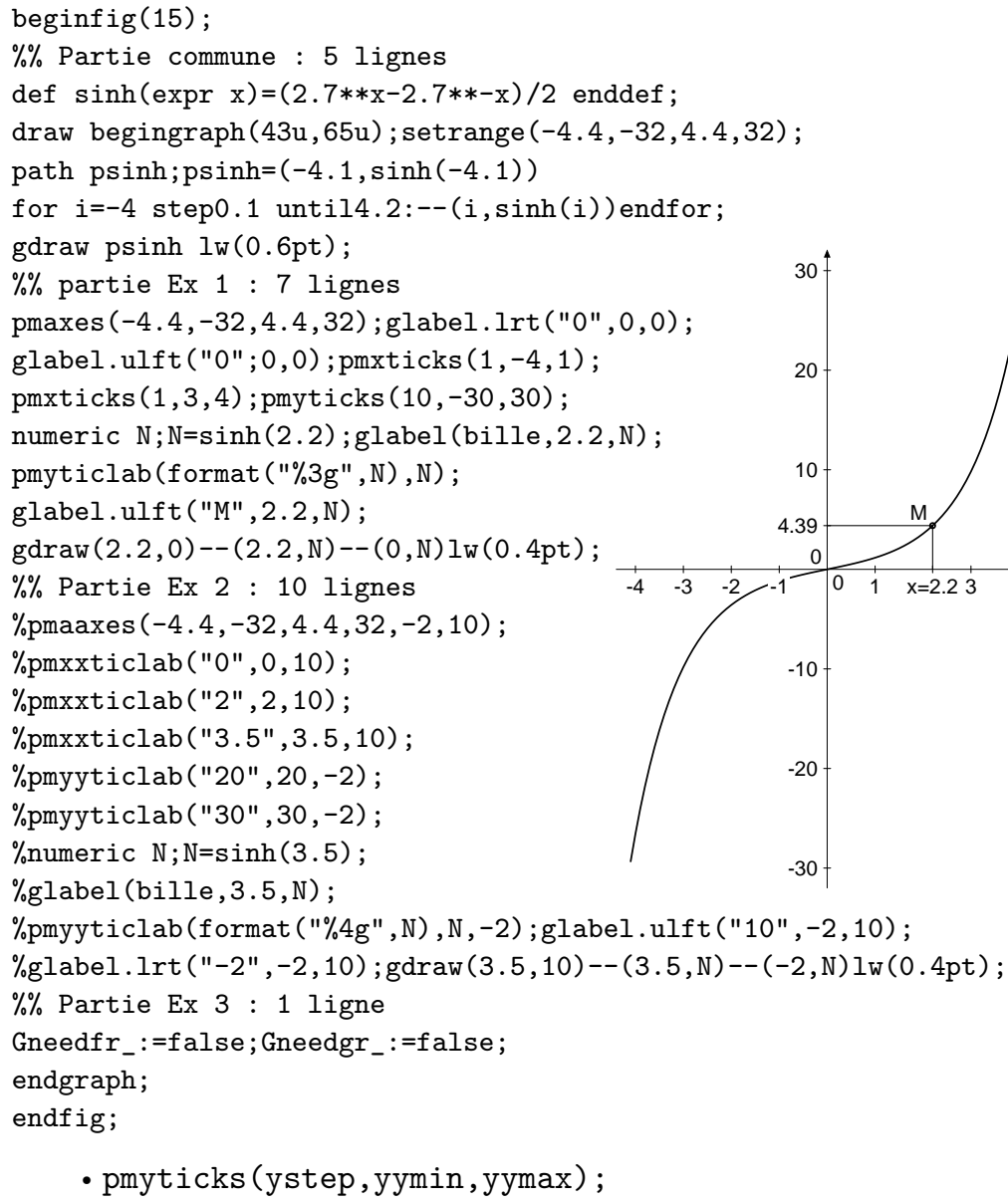

- pmyticks (ystep, yymin, yymax);

trace les graduations de l'axe des ordonnées avec les mêmes notations et les mêmes possibilités. On choisit un pas de 10 et les couples de bornes 
$-30,30$; il faut veiller à ce que la boucle for ... endfor de la macro " tombe" sur la valeur 0 ; on pourrait prendre le pas 0.5 et les bornes $-25,25$ (bien entendu, la symétrie par rapport à 0 n'est pas nécessaire).

- Gneedfr $:=f a l s e ;$ Gneedgr_: =false ;

font respectivement disparaître le cadre et les graduations par défaut.

On a aussi ajouté quelques commandes pour mettre en évidence un point de la courbe avec les lignes de rappel vers les axes. Cela conduit à quelques remarques.

- Les labels sont posés sur un petit rectangle «effacé» pour favoriser leur lecture : dans certains cas, on peut recouvrir parfaitement un label avec un autre label mais la vrai solution consiste à éviter de tracer le label qu'il faudra effacer.

- Pour tracer des graduations supplémentaires ou remplaçant les graduations automatiques ne convenant pas, on utilise les macros pmxticlab (lab, x); et pmyticlab (lab,y);

et, pour tracer de labels supplémentaires, notamment les 0 à l'intersection des axes, on utilise

glabel. suff (lab, $\mathrm{x}, \mathrm{y})$;

où $\mathrm{x}$ et $\mathrm{y}$ sont les abscisses et les ordonnées correspondantes. L'argument lab peut être une chaîne et on écrit alors "ab" ou "3.4" ; il peut aussi être un nombre n préalablement fixé ou calculé par METAPOST auquel cas on écrit alors decimal n, où decimal est une commande qui prend un nombre et donne la chaîne correspondante, ou bien encore [158] format ( $" \% 3 g ", n)$

pour se limiter à 3 chiffres significatifs (ce qui suffit largement pour une figure).

La procédure de remplacement des axes et des graduations a des limites que l'on peut contourner assez facilement.

- Les macros pmxticks et pmxticks ne peuvent pas être utilisées dans le cas de graduations non linéaires (cas des arguments log de la commande setcoords). Il faut alors placer les graduations une par une avec les macros pmxticlab (lab, x); et pmyticlab (lab,y);

- Ces quelques macros élémentaires précédentes ne peuvent pas traiter le cas où les axes ne se coupent pas au point $(0,0)$. Si ce point d'intersection est $(a, b)$, on tracera les axes avec la macro pmaaxes (xmin, $y$ min, $x \max , y \max , a, b)$;

Cahiers GUTenberg no 52-53-Octobre 2009 
et on placera les graduations «à la main » avec les macros pmxxticlab (lab, x, b); et pmyyticlab(lab,y,a);

en remplacement des macros précédentes, partie (Ex 2) de la figure 15. Les commandes glabel et gdraw s'utilisent toujours de la même manière. On peut faire un test en cachant la partie (Ex 1) et en découvrant la partie (Ex 2).

En guise de conclusion de cette section, on peut dire que, si on ne peut pas tout faire, on peut tout de même faire pas mal de choses pédagogiquement valables; sont évidemment exclues toutes les innombrables fioritures qui remplissent certains ouvrages du secondaire et dont on peut discuter l'utilité quand on est face à certains groupes de TD de première année d'université. L'auteur de ce petit document pense que l'idéal serait de reprendre graph.mp en y intégrant au moins les fonctionnalités fournies par graphsup.mp; en effet, il faudrait des macros pour ajouter des ticks sans label et quelques autres petites choses. Ce n'est peut-être pas un tâche énorme : en effet, on peut remarquer que gdraw permet de déplacer les axes «d'origine» mais les graduations au point d'intersection vont se superposer... 



\section{CHAPITRE 7 \\ METAPOST et $\mathrm{T}_{\mathrm{E}} \mathrm{X}$}

Ce chapitre est consacré à l'utilisation conjointe de $\mathrm{TE}_{\mathrm{E}}$ et META$\mathrm{POST}$, plus précisément à l'intégration dans des documents $\mathrm{T}_{\mathrm{E}} \mathrm{X}$ ou $\mathrm{L}_{\mathrm{T}} \mathrm{T}$ de figures faites avec METAPOST et portant des lettrages ${ }^{7}$ composés avec $\mathrm{T}_{\mathrm{E}} \mathrm{X}$ ou $\mathrm{LT}_{\mathrm{E}} \mathrm{X}$. Il y a deux possibilités :

- on fait les figures, on y ajoute les lettrages puis on les intègre dans le document;

- on inclue le code des figures dans le document lui-même et un traitement du document conduit au résultat final de façon transparente.

La deuxième possibilité, initialement utilisable seulement dans le cadre de ConTEXT, est maintenant disponible pour les utilisateurs de LATEX. Bien entendu, cette solution exige l'utilisation d'extensions appropriées. Le lecteur se doute bien qu'un premier traitement va extraire le code des figures qui devra être traité par METAPOST... et qu'un autre traitement va placer la figure à la place demandée (mais grâce à la primitive $\mathrm{T}_{\mathrm{E}} \mathrm{X} \backslash$ write18, on a l'impression qu'il n'y a qu'un seul traitement).

Pour celui qui écrit ces quelques lignes, il semble que, dans le cas d'un auteur assurant lui-même la composition de son ouvrage, la première solution est de loin la plus pratique : qui n'a jamais modifié une figure en cours de réalisation et qui n'a jamais adapté une figure au texte explicatif (ou la réciproque quand la figure est terminée) ? Laissons de côté cette discussion et passons au vif du sujet.

\section{1. ÉTAPES DU LETTRAGE DES FIGURES}

On va créer un document qui est en fait le présent chapitre de ce petit manuel. On commence par créer un fichier de figures figz.mp en

7. Lettrage est utilisé ici pour tout élément de figure exigeant d'être composé avec $\mathrm{T}_{\mathrm{E}} \mathrm{X}$ ou $\mathrm{LAT}_{\mathrm{E}} \mathrm{X}$ : caractères mathématiques, formules, éléments de texte, etc.

METAPOST raconté aux piétons, chap. 7 
reprenant le préambule des fichiers de figure précédents, en y enlevant les trois instructions qui concernent l'utilisation de la fonte Helvetica (sect. 1.3) et en y remplaçant la commande de formatage de composition init_numbers (...) (cf. introduction du chap. 6) par :

init_numbers (btex $\$-\$$ etex, btex $\$ 1$ etex, btex $\$ \backslash$ times $10 \$$ ) etex, btex $\$\{\}^{\wedge}-\$$ etex, btex $\$\{\}^{\sim} 2 \$$ etex $)$

pour avoir une composition correcte de la notation scientifique des grands nombres.

La première figure que l'on fait comprend :

- les axes et un point de coordonnées $x=13 \mathrm{~mm}$ et $y=19 \mathrm{~mm}$,

- le lettrage «cas 1) : $x \in \mathbb{Q}$ » affecté à ce point.

La commande pour ce lettrage est [46] :

label.top(btex cas 1): \$x\in\mathbb $Q \$$ etex, (13u,9u));

La macro label. suff est toujours utilisée: son premier argument est le

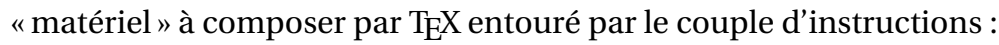
btex ... etex.

Pour que cette composition soit possible, il faut que $\mathrm{T}_{\mathrm{E}} \mathrm{X}$ ait un certain nombre de directives pour faire cette composition. Dans l'exemple choisi, pour avoir le caractère $Q$ ajouré, il faut utiliser l'extension amsmath, etc. On place donc, avant le code des figures les instructions :

verbatimtex

\documentclass $\{$ book\} ou \{article\}

\usepackage \{amsmath, amssymb\}

etex

D’un façon générale, il faut ajouter, entourées par les instructions [48] verbatimtex ... etex;

toutes les commandes que $\mathrm{T}_{\mathrm{E}} \mathrm{X}$ doit utiliser pour composer le «matériel » du lettrage, en particulier, tout ce qui concerne les fontes et leurs codages.

Lorsque l'on fait une figure complexe, il peut être avantageux de faire dans un premier temps la figure sans lettrage et de la tester; à chacun de voir ce qui lui convient le mieux!

\subsection{Production des Fichiers teX et DV I des LetTrages}

Le fichier figz. tex est extrait du fichier figz . mp grâce à un utilitaire nommé mpto [168] :

mpto figz.mp > figz.tex

Cahiers GUTenberg no 52-53-Octobre 2009 
Il contient uniquement la partie $\mathrm{T} \mathrm{E}$ - $\mathrm{LAT}_{\mathrm{E}} \mathrm{X}$ des commandes de lettrages ; il est instructif d'éditer ce fichier figz.tex pour voir le résultat de l'extraction faite par mpto et constater que son traitement par $\mathrm{LT}_{\mathrm{E}} \mathrm{X}$ va donner des «petites boîtes » contenant ces lettrages. Ce traitement se fait par:

latex figz.tex

pour donner le fichier figz.dvi.

\subsection{Production du Fichier MPX DeS Fichiers De Figure LetTrés}

Le fichier de lettrage figz.dvi est alors transformé en un fichier de commandes de bas niveau METAPOST [168] :

dvitompx figz.dvi figz.mpx;

Ce fichier figz . mpx peut être édité et, malgré son aspect peu engageant, on pourra y reconnaître des positionnements, des caractères et les noms $\mathrm{LAT}_{\mathrm{E}} \mathrm{X}$ des fontes utilisées.

On termine par le traitement normal du fichier de figure : mp figz.mp

qui produit les fichiers PostScript figz.1, figz.2, etc. Dans cette opération, lorsque METAPOST rencontre le groupe btex ... etex, il cherche le fichier figz.mpx de même nom et y prend le code METAPOST de bas niveau correspondant aux lettrages pour écrire le code PostScRipt complet des figures.

\subsection{Automatisation des Opérations De LetTRAGe}

Une fois que l'on a compris ce que l'on doit faire, on peut le rendre transparent en définissant par exemple le fichier de commandes mmp.bat

mpto $\% 1$.mp > $\% 1$.tex

latex $\% 1$.tex

dvitompx $\% 1$.dvi $\% 1$.mpx

$\mathrm{mp} \% 1$.mp

qui prend en charge toutes les opérations (utilisation : mmp figz). On constate sur le code page suivante (fichier figz.mp jusqu'au code de la figure 1) que, partout où l'on avait une chaîne de caractères à composer sous la forme "abcde", il faut la remplacer par l'ensemble :

btex (code de composition en $\mathrm{LT}_{\mathrm{E}} \mathrm{X}$ ) etex. 
Figure 1

figz.mp
$\% \% \%$ Fichier de figures figz.mp \% \% \% 1) Preambule

$\%$ Affectations generales et macros generales

$\mathrm{u}=1 \mathrm{~mm} ;$ linejoin :=mitered; ahlength : $=2 \mathrm{pt}$;

prologues : $=1 ; \% \%$ labeloff set $:=2 \mathrm{pt}$;

$\%$ ici initnumbers (cf. deb. chap. avec \scriptstyle dans les \$.\$)

input macutil.mp;

$\%$ Pour boites, liaisons et courbes

input rboxes.mp;input boitesup.mp;

rbox_radius: $=3 \mathrm{pt}$; bboxmargin: $=1 \mathrm{pt}$;

input graph.mp;input grapsup.mp;

$\%$ Directives pour composition

verbatimtex

$\backslash$ documentclass $\{$ book\}

\usepackage [latin1] \{inputenc\}

\usepackage [T1] \{fontenc\}

\usepackage \{amsmath, amssymb\}

$\backslash$ usepackage $\{$ lmodern $\} \backslash$ def $\backslash$ fnsz $\{\backslash$ footnotesize $\}$

etex

$\% \%$ 2) Figures

beginfig(1);

axespapiermilli $(-1.5,-1.5,38.5,26.5)$; drawpt ((13u , 19u ), 1.5pt) ;

label.top(btex \fnsz cas 1): \$a $\backslash$ in $\backslash$ mathbb Q \$ etex, (13u,19u)); endfig;

Reste à savoir si l'on peut voir directement la figure figz.1 correspondant au fichier figz.mp dont le début est ci-dessus. Bien sûr qu'on ne peut pas la voir car ce fichier PostScRIPT ne contient pas les dessins des caractères des fontes utilisées (dessins contenus dans les fichiers .pfa ou .pfb)! Si l'on introduit dans le fichier figz.mp l'instruction prologues : $=1$; ou :=2; au lieu de :=0; alors on peut, en général, voir la figure où chaque caractère est representé par le caractère de la fonte par défaut de même code s'il existe. Pour voir la figure et ses « vrais » lettrages, il faut l'intégrer dans le document. Le fichier PostScRIPT du document complet créé par DVIPs contient les dessins des caractères des fontes utilisées (dessins pris dans les fichiers .pfa ou .pfb), ce qui permet de voir les « vrais» lettrages.

\section{LETTRAGE EN IATEX DE QUELQUES FIGURES}

Sur la page suivante, on reprend les deux figures 3.11 et 5.8 en y introduisant les lettrages "à la $\mathrm{IAT}_{\mathrm{E}} \mathrm{X}$ "; on ne porte sur les listings que les lignes changées.

Cahiers GUTenberg no 52-53 - Octobre 2009 
beginfig (2);

$\% \%$ Rreprise de la figure 3.11

Figure 2

figz.mp

...

label.bot(btex $\$ z_{-}\{0\} \$$ etex, $z 0$ );

label.ulft (btex $\$ z_{-}\{1\} \$$ etex,z1);

label.top(btex $\$ z_{-}\{2\} \$$ etex, $\left.z 2\right)$;

label.bot(btex $\$ z_{-}\{3\} \$$ etex, z3);

label.lft(btex $\$ z_{-}\{4\} \$$ etex, $\left.z 4\right)$;

label.bot (btex $\$ z_{-}\{5\} \$$ etex, z5);

label.rt (btex $\$ \mathrm{p}_{-}\{\mathrm{a}\} \$$ etex, (6.5pt,8.1pt));

label.rt(btex $\$ \mathrm{p}_{-}\{\mathrm{c}\} \$$ etex, $\left.(71 \mathrm{pt}, 8.1 \mathrm{pt})\right) ; z_{0}$

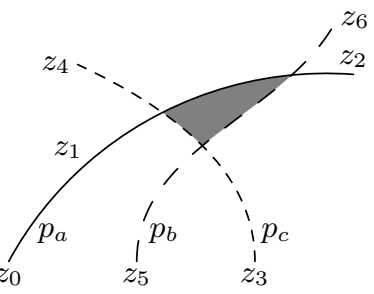

label.rt (btex $\$ p_{-}\{b\} \$$ etex, (38.7pt,8.1pt));

label.rt(btex $\$ z_{-}\{6\} \$$ etex, z6+(0,1.5pt));

endfig;

beginfig(3);

$\% \%$ Rreprise de la figure 5.8

Figure 3

figz.mp

axespapiermilli $(-6.2,-5.5,39.8,34.5)$;

ellit.ka(btex \huge AA etex); tracebe.ka( $(5 u, 0), 0)$;

rboxit.kb(btex \huge aa etex); tracebe.kb((30u,0),0);

cirit.kc(btex filtre etex); tracebe.kc( $(5 u, 13 u), 0)$;

diamit.kd(btex test etex);

tracebe.kd((30u, 15u), 0);

boxit.ke(btex \Large INput etex);

tracebe.ke((30u,30u),0);

..

picture laba,labb;

label.top(btex \tiny voie indirecte etex, $(15 \mathrm{u}, 22.5 \mathrm{u}))$;

laba=thelabel (btex \tiny voie directe etex, $(15 u, 21 u)+0.5 * u p)$;

unfill bbox laba; draw laba;

label.lft(btex \tiny voie

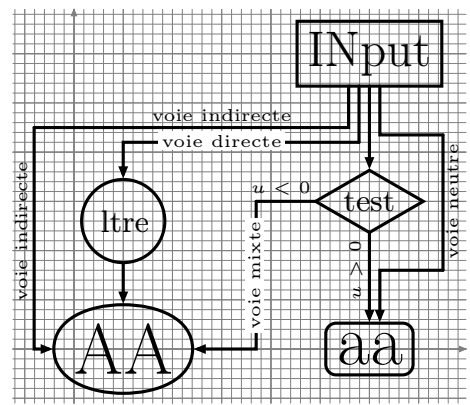
indirecte etex rotated 90 , (xpart ka.w-2u,12u));

label.top (btex \tiny $\$ \mathrm{u}<0 \$$ etex, $(21 \mathrm{u}, 15 \mathrm{u})$ );

label.lft(btex \tiny $\$ \mathrm{u}>0 \$$ etex rotated $90,(30 \mathrm{u}, 8 \mathrm{u})$ ); label.rt (btex

\tiny voie neutre etex rotated 90 , (xpart kd.e+2u,15u)+left);

labb=thelabel (btex \tiny voie mixte etex rotated 90 ,

$(18.5 u, 7.5 u)+0.5 * l$ eft); unfill bbox labb; draw labb;

endfig;

Pour la figure 3.11 , on constate que l'on a du corriger la position des noms de deux des chemins car le pied de la lettre italique $p$ avait tendance a recouvrir le chemin lui-même.

Pour la figure 5.8, on a aussi fait des corrections car, dans le cas particulier de cette figure, on a des textes sans lettre à pied et avec 
très peu de lettres à hampe; le résultat est que le texte vertical "voie indirecte" semble plus près de la liaison que le texte "voie neutre".

Ce type de correction est la plupart du temps superflu mais on peut rencontrer des cas où une intervention est souhaitable (cas de caractères mathématiques avec exposants ou indices, par exemple). La correction apparaît d'autant plus nécessaire que l'on diminue la valeur de labeloff set; cependant, une faible valeur améliore, aux yeux de l'auteur de ces lignes, la qualité des figures; à chacun de choisir!

L'intégration de ces figures dans un document $\mathrm{L} \mathrm{TEX}$ se fait normalement avec l'extension graphicx (voir début du chapitre 1). Il est signalé que, si le document est composé avec pdflatex [171], on peut obtenir directement le fichier .pdf ; mais cela nécessite un peu de préparation.

Figure 4 figz.mp

\section{LETTRAGE EN LATEX DE QUELQUES GRAPHIQUeS}

Sur cette page et la suivante on reprend directement les figures 6.8 (pas de modification nécessaire) et 6.11 (modification du codage des trois chaînes de caractères). On constate que la taille obtenue par l'introduction de \scriptsize dans la macro init_numbers est suffisante.

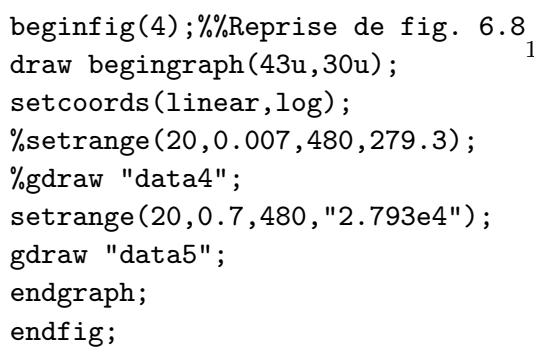

On termine en reprenant la figure 15 qui présente des difficultés résultant des principes mêmes de la méthode de production des lettrages. On rappelle que l'on doit remplacer toutes les occurences de chaînes du type "abcd" par des ensembles du type btex (code de la composition en ${ }_{\mathrm{L}} \mathrm{TEX}$ ) etex. Mais les macros qui tracent les graduations, pmxticlab par exemple, contiennent des boucles du type suivant :

for $i=x m i n . . . . . . g l a b e l$ (decimal $i, i, 0)$...endfor;

Cahiers GUTenberg no 52-53-Octobre 2009 


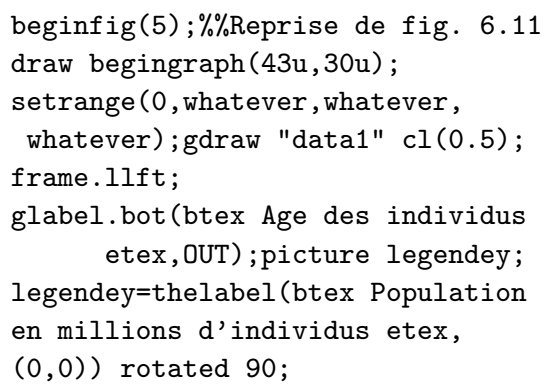

Il faudrait donc introduire des ensembles btex... etex dans cette macro; en effet on se rend compte que decimal i y joue le rôle d'une chaîne du type "abcde" qui doit être remplacée suivant la prescription. Mais, pour extraire les lettrages à composer, mpto repère les couples btex. . . etex, il ne peut pas voir ceux qui sont dans la macro : il ne voit que le nom de la macro et passe à la suite; les ensembles btex. . . etex ne peuvent pas être dans une macro ... et c'est pour cela que, si cette prescription n'est pas respectée, la compilation donne un message d'erreur du type :

" commande interdite dans la définition etc.»

En conséquence, pour les graduations des axes on va garder les définitions contenant des boucles dans lesquelles on ne touche rien; par contre on va ajouter dans le prologue les commandes :

defaultfont: $=$ "CMR10"; et defaultscale $:=0.5$;

Ce choix (forcé) ne perturbe pas la qualité de la figure comme on peut le constater sur la page suivante : le seul reproche que l'on peut faire concerne le point décimal (au lieu de la vigule). Ici, les tailles sont données à titre indicatif; sur une figure plus grande, on pourrait choisir le facteur d'échelle 0.9 , ce qui correspond à la taille par défaut small du préambule. Par contre, les chaînes de caractères sont traitées comme cela a été expliqué. 
Figure 6

figz.mp beginfig(6);\% $\%$ Reprise de fig. 6.15

$\% \%$ Partie commune

def $\sinh (\operatorname{expr} \mathrm{x})=(2.7 * * \mathrm{x}-2.7 * *-\mathrm{x}) / 2$ enddef;

draw begingraph ( $43 \mathrm{u}, 65 \mathrm{u})$; setrange $(-4.4,-32,4.4,32)$;

path psinh,pcosh;

$\operatorname{psinh}=(-4.1, \sinh (-4.1))$

for $i=-4$ step 0.1 until $4.2:--(i, \sinh (i))$ endfor;

gdraw psinh $1 \mathrm{w}(0.6 \mathrm{pt})$;

$\%$ partie Ex 1 : 11 lignes

pmaxes $(-4.4,-32,4.4,32)$; glabel.lrt $(" 0 ", 0,0)$;

defaultfont: $=$ "CMR7 "; defaultscale $:=0.9$;

pmxticks $(1,-4,1)$; pmxticks $(1,3,4)$;

pmyticks $(10,-30,30)$;

glabel.Irt ("0",0,0); glabel.ulft ("0" ,0,0);

pmxticlab (btex \kern-6pt $\$ \mathrm{x}=2.2 \$$ etex, 2.2$)$;

numeric $\mathrm{N} ; \mathrm{N}=\sinh (2.2)$;

glabel (bille, 2.2,N);

pmyticlab (format ( $1 \% 3 g ", N), N)$;

glabel.ulft (btex $\$ \backslash$ mathrm $M \$$ etex, $2.2, \mathrm{~N}$ );

$\operatorname{gdraw}(2.2,0)--(2.2, N)--(0, N) I_{w}(0.4 p t)$;

$\%$ Partie Ex 2 : 10 lignes

$\%$ defaultfont $:=$ "CMR7" ; def aultscale $:=0.8$;

$\%$ pmaaxes $(-4.4,-32,4.4,32,-2,10)$;

$\%$ pmxxticlab ("0" , 0, 10);

$\%$ pmxxticlab ("2" , 2, 10);

\%pmyyticlab("20" , 20, -2) ; pmyyticlab ("30" , 30, -2);

$\%$ numeric $\mathrm{N} ; \mathrm{N}=\sinh (3.5)$; glabel (bille, $3.5, \mathrm{~N})$;

$\%$ glabel.ulft ("10" , -2,10); glabel.lrt ("-2" , -2, 10);

\%defaultscale: $=0.9$; pmxxticlab ("3.5" $, 3.5,10)$;

\%pmyyticlab ("16.5" , N , -2) ; (format ("\%3g", N) , N , -2);

$\% \operatorname{gdraw}(3.5,10)--(3.5, \mathrm{~N})--(-2, \mathrm{~N})]_{\mathrm{w}}(0.4 \mathrm{pt})$;

$\%$ Parie Ex 3 : 1 ligne

Gneedfr $r_{-}:=$false; Gneedgr $:=f a l s e$;

endgraph;

endfig;

\section{METAPOST ET T $T_{E} X$, TOUT À LA FOIS !}

Comme l'auteur de ces lignes ne pense pas qu'un débutant puisse se lancer dans la composition d'un long document avec de nombreuses figures en suivant cette méthode [172-175], il est simplement donné sur la page suivante un petit exemple que l'on peut facilement tester dans un autre répertoire que celui des exercices précédents. 


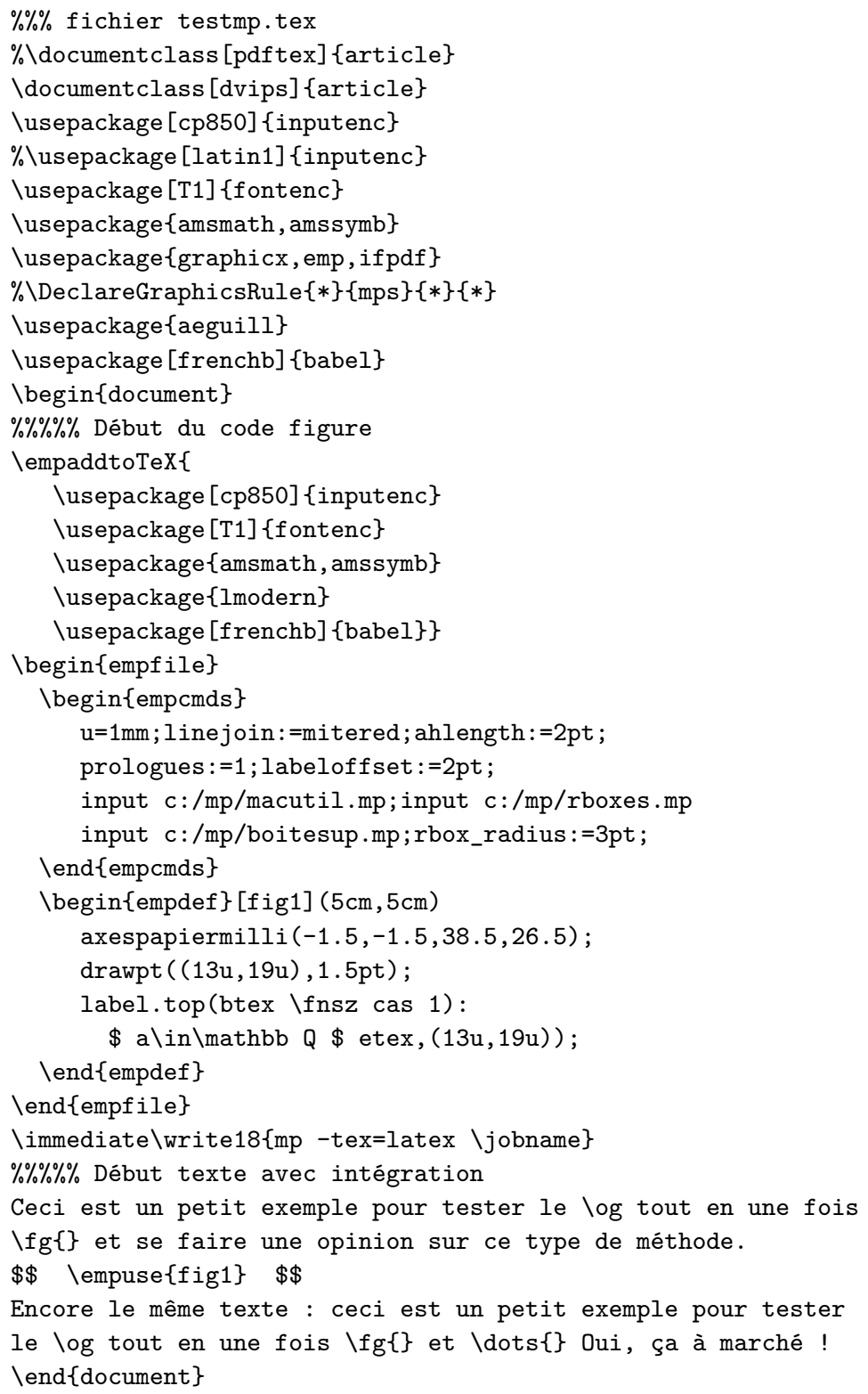


Le traitement se fait en donnant l'option autorisant l'utilisation de la primitive $\mathrm{T}_{\mathrm{E}} \mathrm{X} \backslash$ write18:

latex -shell-escape testmp

Le fichier ci-dessus correspond au traitement par LATEX et Dvips. La même chose est également possible pour un traitement par PDFTEX [F 172-174].

Cahiers GUTenberg no 52-53-Octobre 2009 


\section{Conclusion}

Avant la conclusion proprement dite, on signale qu'il y a de très nombreuses extensions disponibles pour METAPOST; elles sont en grande majorité très spécialisées. C'est à chacun de savoir si, pour une tâche limitée (et exceptionnelle), il est plus rentable d'écrire quelques macros (peut-être pas très bien optimisées) que de se plonger dans le manuel d'une extension avec le risque de découvrir après des essais qu'elle ne convient pas du tout. Un chercheur de théorie des particules peut valablement se plonger dans l'extension FEYNMAN, mais pour illustrer un paragraphe d'un article avec un diagramme de Feynman, il est bien plus avantageux de le créer directement (en s'aidant d'un modèle pour respecter les conventions traditionnelles). Voici une liste non exhaustive d'extensions suivies d'une très succincte description :

textpath : écriture le long d'une courbe, venn : diagramme de Venn, threed: dessin en $3 \mathrm{D}$, splines : tracé de splines, slidesshow : transparents de présentation, piechardmp : camemberts pour statisticiens, misc : objets de base en $3 \mathrm{D}$, mfpic: macros générales; liste de couleurs (en CMJN), metaobj : "pstricks écrit en METAPOST", latexmp : macros de gestion (type etex...btex), hatching : fonds hachurés, feynmf : diagrammes de Feynman, featpost : figures complexes (contributions multiples), exteps : pour compléter en surcharge des .eps, metaULM : nœuds et liaisons inter-nœuds, cmarrows : flèches, accolades, etc., 
mppatern : motifs de remplissage, metaplot : tracé de graphiques, makecirc : dessin de circuits électroniques, m3d : animation en $3 \mathrm{D}$, expressg : organigrammes élémentaires, graph : tracé de graphiques (cf. chap. 6), graphsup : quelques macros complémentaires à graph (cf. chap. 6), config : fichier d'initialisation de METAPOST.

En outre, ConTEXT contient des modules utilisables dans l'environnement LATEX; par exemple, le module METAFUn permet d'obtenir des remplissages dégradés allant d'une couleur à une autre en passant par une troisième.

Comme on peut le constater, il est parfois difficile de faire un choix; par contre, on peut signaler que mf pic contient de nombreuses macros très utiles, fonctions trigonométriques inverses par exemple, (fichier grafbase.mp), ainsi que les définitions des 64 couleurs de la table de Hafner (fichier dvipsnam .mp).

Il semble évident que l'utilisation de METAPOST demande plus d'investissement que l'utilisation de TikZ; cela parce que METAPOST est une vrai langage avec sa propre syntaxe alors que TikZ est un outil de dessin d'objets graphiques intégré dans le «monde» $\mathrm{LT}_{\mathrm{E}} \mathrm{X}$ (bien qu'ayant une syntaxe spécifique en ce qui concerne les paramètres et les options des commandes) ; la dernière phrase du premier paragraphe de l'introduction précise le sens de ce commentaire : TikZ dessine, METAPOST construit.

On ne peut en dire plus car la personnalité de l'utilisateur potentiel joue un rôle important. C'est pour cela que l'auteur de ces quelques lignes a voulu présenter METAPOST d'une manière telle que, assez rapidement (autrement dit en langage du jour, sans trop perdre de temps), on puisse découvrir METAPOST avec ses avantages et ses inconvénients; il espère y avoir réussi !

Pour terminer, le lecteur pourra reproduire l'illustration de la couverture (il faut charger le fichier grafbase.mp pour disposer des fonctions arcsin et arctan). La théorie de l'arc-en-ciel, outre la décomposition montrée sur la figure, fait appel à un lourd calcul de diffraction.

(I Yves Soulet

ysoulet@cict.fr

Cahiers GUTenberg no 52-53-Octobre 2009 


\section{Index}

$\% 3 g, 98$

$\% 4 \mathrm{~g}$ (format -), 95

$\%<, 13$

$\% \mathrm{~g}$ (format -), 95

$\%$ pg \%pe (formats -), 94

$\&, 44,53,54$

$*, 49-51$

$* *, 49$

$+, 49-51$

$++, 49$

$-, 49-51$

$--, 17$

-shell -escape, 110

.., 29

$\ldots, 35$

$/, 49-51$

$<, 49,54$

$<=, 49,54$

$<>, 49-51,54$

$=, 49-51,54$

$>, 49,54$

$>=, 49,54$

abs, 49,50

addtocurrentpicture also, 56

ahangle, 23

ahlength, 23, 58

and, 55

angle, 50

arcd, 27

arclength, 43, 54

arctime, 54 arctime of, 43

ASCII, 54

augment.p, 86

autogrid, 93

autogrid.lft -bot -rt, 90

axespapiermilli, 11

bb.c, 66

$\mathrm{bb} . \mathrm{dx}, 66$

bb.dy, 66

bb.n -e -s -w, 66

$\mathrm{bb} . \mathrm{ne}-\mathrm{se}-\mathrm{sw}-\mathrm{nw}, 66$

bb.off, 66

bbox, 78

bboxmargin, 65,78

bbtrace, 72,73

beginfig, 11, 48

begingroup, 44

beveled, 21

black, 24

blue, 24

bluepart, 51

boitessup.mp, 65

boitesup.mp, 70

boolean, 49

boxes.mp, 60

boxit.bb, 66

bpath.bb, 69

btex ... etex, 102, 107

btrace, 71

buildcycle, 39, 54

butt, 21 
ceiling, 49

char, 54

chemin, 13, 26

circit, 70

circleit, 70

clip, 17, 18

color, 49

commandes les plus utilisées, 59

concaténation de chemins, 44

controls and, 31

cosd, 49

couleur, 26

courbe de Bézier, 31

courbure près d'un point guide, 34

création des figures, 12

curl, 34

currentpicture, 56

currentpicture, 18, 58

cutafter, $43,54,73$

cutbefore, 43, 54, 73

cyan, 24

cycle, 14, 29

découper une partie d'une figure, 17

dashed evenly scaled, 22

dashed withdots scaled, 22

dashpattern, 23

débogage, 48,59

decimal, 98

déclaration, 48

déclaration des variables, 26

def ... enddef, 27

defaultdx, 66

defaultdy, 66

defaultf ont, 15, 107

defaultpen, 19

defaultscale, 15, 107

dessin, 18, 26

diamit.bb, 70

dir, 50

direction de la tangente (donner la

$$
-), 32
$$

direction of, 40,54

directiontime of, 41,54

div, 49

donnees.bb, 72

dotlabel, 85

down, 74

draw, 11, 13, 17

draw begingraph, 82

drawarrow, 23, 73, 74

drawboxed, 66

drawdbarrow, 23

drawoptions, 19, 20

drawpt, 20

drawunboxed, 69

droite (représentation d'une -, 25

dvitompx, 103

effacement, 18

ellit.bb, 70

end, 11

endfig, 11

endgraph, 82

endgroup, 44

epsilon, 38

exitif, 63

exitunless, 63

expr, 27, 62

facteur d'échelle, 12

fermeture d'un chemin, 29, 38, 45

figa.mp, 11, 13, 15

figb.mp, 15, 17

figc.mp, 15

figd.mp, 15

fige.mp, 65, 69

figf.mp, 81

figz.dvi, 103

figz.mp, 101, 102

figz.mpx, 103

figz. tex, 102

fill, 17

fin. bb, 73 


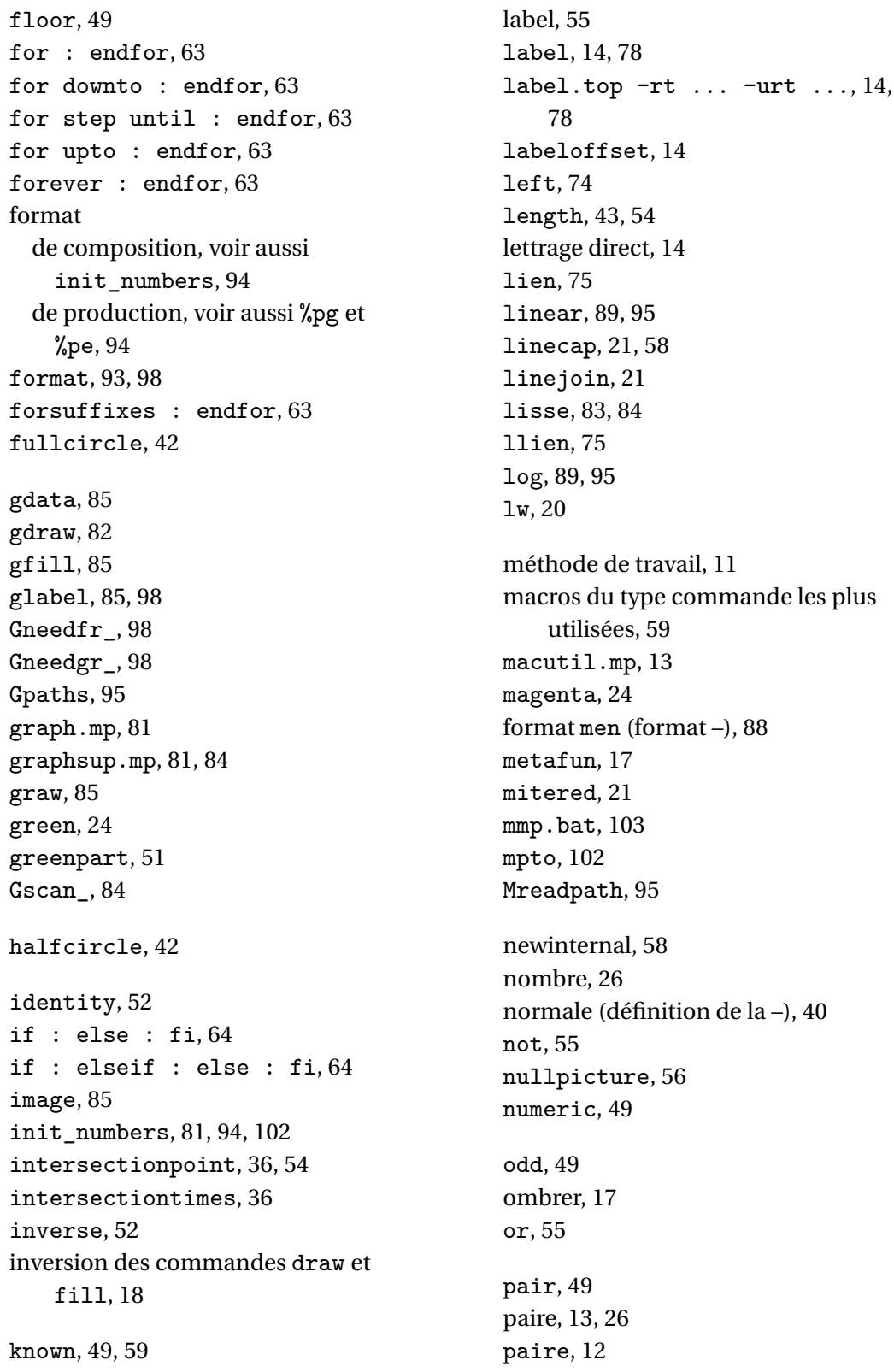




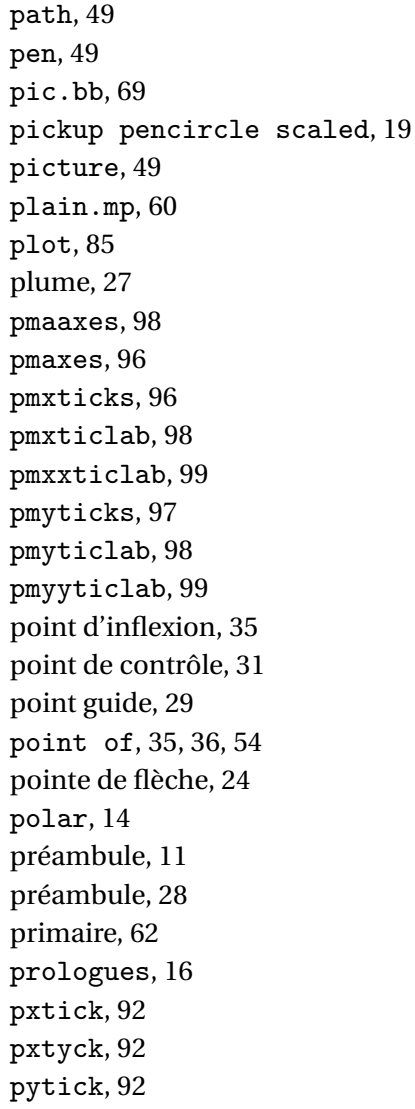

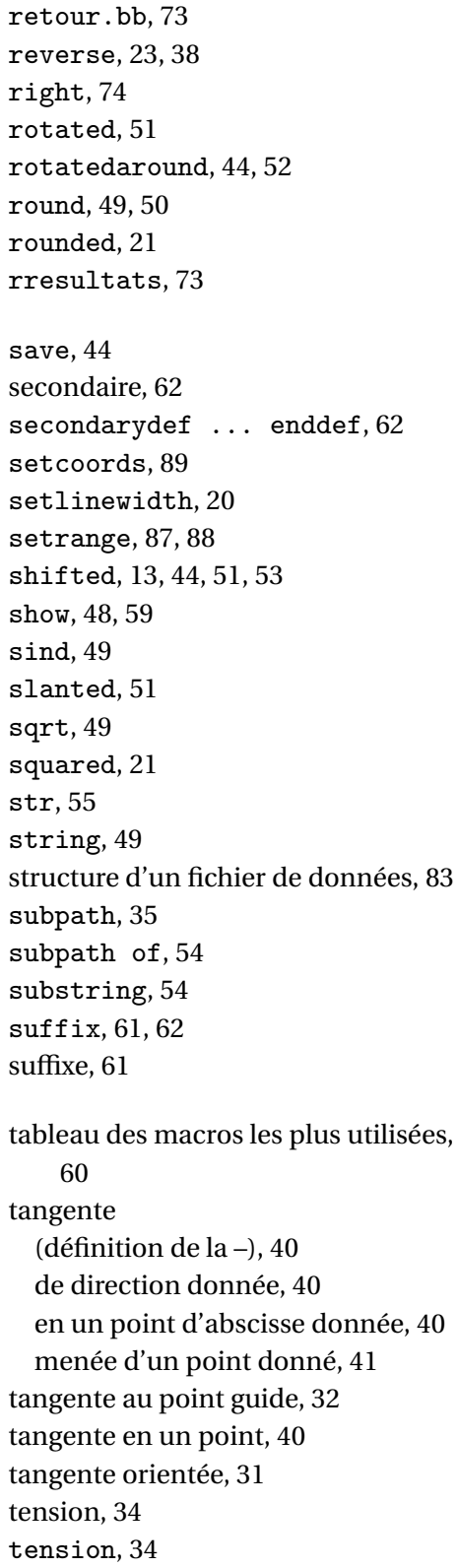

tableau des macros les plus utilisées, 60

tangente

(définition de la-), 40

de direction donnée, 40

en un point d'abscisse donnée, 40

menée d'un point donné, 41

tangente au point guide, 32

tangente en un point, 40

tangente orientée, 31

tension, 34

tension, 34 
tertiaire, 62

test.bb, 73

text, 27, 61, 62

thelabel, 84, 85

thelabel.top -rt ... -urt .., 78

tracé de lignes brisées, 17

tracé de lignes courbes, 29

tracebe, 72

tracebf, 72

transf orm, 49

transformation, 13, 27

transformation EPS-PDF, 12

transformed, 52

u, 12

undraw, 17, 18

unfill, 17, 18

unit vector, 50

unités, 12

up, 74

vardef $\ldots$ enddef, 62 variable interne, 18,27

variable tableau, 14

verbatimtex ... etex, 102

visualisation des figures, 12

whatever, 26, 88

white, 24

withcolor, 24

withpen pencircle scaled, 19, 57

\write18, 101, 110

$\mathrm{x}[], 14,48$

xpart, 25,50

xscaled, 52

y [], 14, 48

yellow, 24

ypart, 25, 50

yscaled, 52

$z$ [] $, 13,48$

zscaled, 52 